Foundations and Trends ${ }^{\circledR}$ in

Networking

Vol. 3, No. 2 (2008) 127-248

(C) 2009 M. Haenggi and R. K. Ganti

DOI: $10.1561 / 1300000015$

\title{
Interference in Large Wireless Networks
}

\author{
By Martin Haenggi and Radha Krishna Ganti \\ Contents
}

1 Introduction

128

1.1 Interference Characterization

1.2 Signal-to-Interference-Plus-Noise

Ratio and Outage

132

2 Interference in Regular Networks

134

2.1 General Deterministic Networks 134

2.2 One-Dimensional Lattices

135

2.3 Two-Dimensional Lattices 140

2.4 Outage 144

3 Interference in Poisson Networks 147

3.1 Shot Noise 148

3.2 Interference Distribution 149

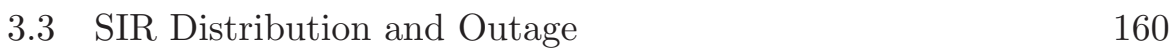

$\begin{array}{lll}3.4 & \text { Extremal Behavior } & 161\end{array}$

3.5 Power Control 162

3.6 Spread-Spectrum Communication 168

3.7 CSMA and Interference Cancellation 169

3.8 Interference Correlation 174 
4 Interference in Poisson Cluster Networks 186

4.1 Interference Characterization 190

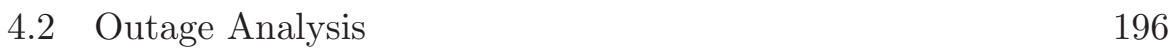

5 Interference in General Motion-Invariant Networks 199

5.1 System Model 199

5.2 Properties of the Interference 201

5.3 Bounds on the Interference Distribution 203

5.4 Asymptotic Behavior of the Interference Distribution 209

5.5 Examples and Simulation Results 218

6 Conclusions 223

A Mathematical Preliminaries $\quad 227$

A.1 Point Process Theory 227

A.2 Palm Distributions 236

A.3 Stable Distributions 240

$\begin{array}{ll}\text { Acknowledgments } & 242\end{array}$

$\begin{array}{ll}\text { Notations and Acronyms } & 243\end{array}$

References $\quad 245$ 
Foundations and Trends ${ }^{\circledR}$ in

Networking

Vol. 3, No. 2 (2008) 127-248

(C) 2009 M. Haenggi and R. K. Ganti

DOI: $10.1561 / 1300000015$

\title{
Interference in Large Wireless Networks
}

\section{Martin Haenggi ${ }^{1}$ and Radha Krishna Ganti ${ }^{2}$}

1 Department of Electrical Engineering, University of Notre Dame, Notre Dame, IN 46556, USA, mhaenggi@nd.edu

2 Department of Electrical Engineering, University of Notre Dame, Notre Dame, IN 46556, USA, rganti@nd.edu

\begin{abstract}
Since interference is the main performance-limiting factor in most wireless networks, it is crucial to characterize the interference statistics. The two main determinants of the interference are the network geometry (spatial distribution of concurrently transmitting nodes) and the path loss law (signal attenuation with distance). For certain classes of node distributions, most notably Poisson point processes, and attenuation laws, closed-form results are available, for both the interference itself as well as the signal-to-interference ratios, which determine the network performance.

This monograph presents an overview of these results and gives an introduction to the analytical techniques used in their derivation. The node distribution models range from lattices to homogeneous and clustered Poisson models to general motion-invariant ones. The analysis of the more general models requires the use of Palm theory, in particular conditional probability generating functionals, which are briefly introduced in the appendix.
\end{abstract}




\section{1}

\section{Introduction}

Due to the scarcity of the wireless spectrum, it is not possible in large wireless networks to separate concurrent transmissions completely in frequency. Some transmissions will necessarily occur at the same time in the same frequency band, separated only in space, and the signals from many undesired or interfering transmitters are added to the desired transmitter's signal at a receiver. This interference can be mitigated quite efficiently in systems with centralized control, where a base station or access point can coordinate the channelization and the power levels of the individual terminals, or where sophisticated multi-user detection or interference cancellation schemes can be implemented. However, many emerging classes of wireless systems, such as ad hoc and sensor networks, mesh networks, cognitive networks, and cellular networks with multihop coverage extensions, do not permit the same level of centralized control but require a more distributed resource allocation. For example, channel access schemes are typically based on carrier sensing, and power control is performed on a pairwise rather than a network-wide basis, if at all. In these networks, interference is not tightly controllable and subject to considerable uncertainty. Consequently, interference is the main performance-limiting factor in most 
emerging wireless networks, and the statistical characterization of the interference power becomes critical.

In this monograph, we derive results for the interference statistics in large wireless networks that are subject to one or several sources of randomness, including the node distribution, the channel access scheme, and the channel or fading states. There are two main factors that shape the interference: First, since interfering signals are only separated in space, the spatial distribution of the concurrently transmitting nodes; second, since the amount of interference caused depends on the signal attenuation with distance, the path loss law. The first factor consists of two parts, the node distribution on the one hand and the channel access scheme (MAC) on the other. It is their combination that determines the distribution of transmitting nodes. For example, even if the nodes are very randomly distributed, a good MAC scheme will ensure a certain spacing between concurrent transmitters or, better, between receivers and interferers; hence the distribution of the transmitters at any given moment may be fairly regular. Since the performance of a network is determined by the signal-to-interference-and-noise ratios (SINRs) or, in the pure interference-limited case, by the signal-to-interference ratios (SIRs), the SIR distributions are also derived, usually in the form of outage probabilities $\mathbb{P}(\operatorname{SINR}<\theta)$, which correspond to the cumulative distributions.

The exact characterization of the interference or SIRs for general node distributions and MAC schemes is a very challenging problem. Since our focus in this monograph is on analytical results and on the underlying mathematical techniques, the network models are partly chosen for their tractability, not necessarily because they are the most realistic ones. The analytical methods are best illustrated when applied to simple models, and the results derived will provide bounds for more elaborate ones, in particular when the models considered are in some sense extreme, such as lattice networks on one end and "completely spatially irregular" networks (Poisson networks) on the other. Also, general design principles and guidelines can be inferred more easily from analytical results, and it is our hope the analytical techniques are described in enough detail to enable the reader to apply them to other types of networks. 
We restrict ourselves to the statistics of the (aggregate) interference power when the sources of randomness include the node distribution, the fading states of the channels, and the channel access scheme. We will not be discussing the amplitude statistics of the interference, which depend strongly on the type of signaling employed and may, conditioned on the power, be well approximated by a Gaussian or not [22]. With Gaussian codebooks, the interference amplitude is certainly conditionally Gaussian, and if it is treated as noise at the receiver, its variance or power is the relevant statistic for the achievable link performance. While not optimum in general, treating interference as noise is, in fact, optimum in the Gaussian weak interference or noisy interference regime [42]. In this regime, sophisticated multi-user detectors do not perform better than simple single-user detectors, and the expected value of $\log _{2}(1+$ SINR) is the actual (bandwidth-normalized) capacity.

This monograph is organized as follows:

Section 2 derives the interference for networks with deterministic node placement, in particular lattices. Section 3 is devoted to Poisson networks, where the nodes are distributed as a Poisson point process (PPP). The PPP model is by far the most popular, thanks to its analytical tractability. It lends itself for extended analyses, including the impact of power control and spread-spectrum and interference cancellation techniques, and the derivation of interference correlation coefficients. The following two sections provide generalizations to the Poisson model. In Section 4, the interference properties in clustered Poisson networks are studied, while Section 5 is devoted to general motioninvariant node distributions.

Sections 2 and 3 only require a basic knowledge in probability, while the results in Sections 4 and 5 were obtained using Palm theory, in particular conditional probability generating functionals. The appendix provides a brief introduction of the mathematical techniques used in this monograph.

The results and analytical techniques derived in this monograph will hopefully serve as guidelines for the design of large wireless systems with random user locations. They provide answers to such questions as how the interference statistics and outage probabilities are affected by the user density and distribution, the path loss law, the fading statistics, 
and power control. In turn, given system constraints such as outage or rate requirements, they permit the tuning of the network parameters for optimum performance.

\subsection{Interference Characterization}

The main quantity of interest is the (cumulated) interference. Measured at a point $y \in \mathbb{R}^{d}$ it is given by

$$
I(y)=\sum_{\mathrm{x} \in \mathcal{T}} P_{\mathrm{x}} h_{\mathrm{x}} \ell(\|y-\mathrm{x}\|),
$$

where $\mathcal{T} \subset \mathbb{R}^{d}$ denotes the set of all transmitting nodes, $P_{\times}$the transmit power of node $\mathrm{x}, h_{\mathrm{x}}$ the (power) fading coefficient, and $\ell$ the path loss function, assumed to depend only on the distance $\|y-\mathrm{x}\|$ from node $\mathrm{x}$ to the point $y$.

In a large wireless system, the unknowns are $\mathcal{T}, h_{\mathrm{x}}$, and perhaps $P_{\mathrm{x}}$. The locations of the interfering nodes, together with the path loss law, determine the interference to first order. The impact of fading is smaller but certainly non-negligible, as we shall see. So, in essence, it is the network geometry or, more precisely, the interference geometry, that determines the distribution of the interference. The geometry consists of the underlying node distribution that, together with the channel access scheme, determines the locations of the interfering nodes, and the path loss law, which determines the strength of the interfering power given the distance.

The nodes may be arranged deterministically, for example in a lattice, or in a random fashion, in which case the uncertainty in the nodes' locations is usually represented by a stochastic point process $\Phi$ on $\mathbb{R}^{2}$ or $\mathbb{R}^{3}$ or a subset thereof. Assuming that the point process is simple, i.e., there are no two nodes at the same position, we can write the point process as a random set, $\Phi=\left\{\mathrm{x}_{1}, \mathrm{x}_{2}, \ldots, \mathrm{x}_{N}\right\}$, where the (possibly random) total number of nodes $N$ may be finite or infinite. At any moment in time, the MAC scheme selects a subset of nodes as transmitters. This makes $\mathcal{T}$ in (1.1) and, in turn, the interference, time dependent. In some cases, the interference is stationary, both in time and space, so neither a time index nor a spatial location needs to be specified, and we can simply talk about the distribution of the interference $I$. 
Throughout this monograph, unless otherwise specified, we will assume unit transmit powers at all nodes and the fading to be iid with $\mathbb{E}(h)=1$.

\subsection{Signal-to-Interference-Plus-Noise Ratio and Outage}

\subsubsection{Definitions}

The performance of a wireless network critically depends on the signalto-interference-plus-noise (SINR) levels at the receivers.

Definition 1.1(Signal-to-interference-plus noise ratio (SINR)). The SINR for a receiver placed at the origin $o$ in the two- or threedimensional Euclidean space is

$$
\operatorname{SINR}=\frac{S}{W+I}
$$

where $S$ is the desired signal power, $W$ is the noise power, and $I$ the interference power given by (1.1).

For a fixed modulation and coding scheme and with interference treated as noise, e.g., by using a simple linear receiver, a well accepted model for packetized transmissions is that they succeed if the SINR exceeds a certain threshold $\theta$. So we define the success probability as follows:

Definition 1.2 (Transmission success probability).

$$
p_{s}(\theta)=\mathbb{P}(\operatorname{SINR}>\theta) .
$$

Its complement $1-p_{s}$ is the outage probability, which is the same as the cumulative distribution function (CDF) of the SINR, and we may express the achievable rate (with interference treated as noise) of a link as

$$
\mathbb{E} \log _{2}(1+\mathrm{SINR})=-\int \log _{2}(1+x) \mathrm{d} p_{s}(x)
$$


assuming that the interference amplitude is Gaussian. In the weakinterference regime, this expression is the actual bandwidth-normalized capacity [42].

\subsubsection{Outage in Rayleigh Fading}

In the case of Rayleigh fading, the desired signal power $S$ is exponentially distributed. Assuming $\mathbb{E} S=1$,

$$
p_{s}(\theta)=\mathbb{P}(S>\theta(W+I))=\underbrace{\exp (-\theta W)}_{p_{s}^{W}} \cdot \underbrace{\exp (-\theta I)}_{p_{s}^{I}},
$$

which shows that the success probability is the product of two factors, a noise term $p_{s}^{W} \triangleq \exp (-\theta W)$ that does not depend on the interference, and an interference term $p_{s}^{I} \triangleq \exp (-\theta I)$ that does not depend on the noise. This allows a significant simplification of outage analyses since the joint impact of noise and interference is captured by the product of the success probabilities in the noiseless and the interference-free cases. Moreover, since $\exp (-\theta I)$ is the Laplace transform of the interference evaluated at $\theta$, i.e.,

$$
p_{s}^{I}(\theta)=\left.\mathcal{L}_{I}(s)\right|_{s=\theta},
$$

the interference component of the success probability can be calculated by determining the Laplace transform of $I$, as was noted in $[3,31,54]$. It turns out that this is easier in many cases than determining the distribution. In other words, the SIR distribution when $S$ is Rayleigh fading is known for more types of networks than the distribution of just the interference itself. 


\subsection{General Deterministic Networks}

We would like to calculate the interference measured at the origin in the presence of $n$ interferers at distances $r_{i}>0$ that are active with probability $p$ independently of each other (ALOHA). The path loss law is the standard power law (with normalized distances $r$ ) $\ell(r)=r^{-\alpha}$, and the channels are subject to Rayleigh (amplitude) fading and thus exponential power fading. Hence the power $P_{\mathrm{r} i}$ received from interferer $i$, given that it is transmitting, is distributed exponentially with mean $r_{i}^{-\alpha}$, i.e., the probability density function (PDF) of $P_{\mathrm{r} i}$ is

$$
f_{P_{\mathrm{r} i}}(x)=r_{i}^{\alpha} \exp \left(-r_{i}^{\alpha} x\right), \quad x \geq 0 .
$$

The total interference is

$$
I_{n}=\sum_{i=1}^{n} B_{i} P_{\mathrm{r} i},
$$

where the random variables $B_{i}$ are iid Bernoulli with parameter $p$. 
The Laplace transform of an exponential random variable with mean $1 / y$ is $\mathcal{L}(s)=y /(y+s), s \geq 0$, so we have

$$
\begin{aligned}
\mathcal{L}_{I_{n}}(s) & =\prod_{i=1}^{n}\left(\frac{p r_{i}^{\alpha}}{r_{i}^{\alpha}+s}+1-p\right) \\
& =\prod_{i=1}^{n}\left(1-\frac{p}{1+r_{i}^{\alpha} / s}\right), \quad s \geq 0 .
\end{aligned}
$$

If the number of nodes $n$ is infinite, then the question whether $I_{n}$ has a proper or defective distribution needs to be addressed. By continuity and monotonicity, $\mathcal{L}_{I_{n}}(s)$ converges for all sequences $r_{i}$ if $s>0$, but the corresponding distribution may be defective, i.e., $\mathbb{P}\left(I_{\infty}=\infty\right)=1$. Thanks to the uniform convergence of (2.3), limit and product may be interchanged, and it follows that (2.3) converges to some positive limit for any $s>0$ if and only if

$$
\sum_{i=1}^{\infty} \frac{p}{1+r_{i}^{\alpha} / s}<\infty(\forall s>0) \Longleftrightarrow \sum_{i=1}^{\infty} \frac{p}{r_{i}^{\alpha}}<\infty .
$$

This is the condition for $I_{\infty}$ to have a proper distribution. On the other hand, if

$$
\sum_{i=1}^{\infty} \frac{p}{r_{i}^{\alpha}}=\infty
$$

the interference is infinite a.s.

For example, in the case of a one-dimensional (one-sided) grid with $r_{i}=i, i \in \mathbb{N}$, and $p>0$, the interference is finite a.s. for $\alpha>1$ and infinite a.s. for $\alpha \leq 1$ since the Riemann zeta function $\zeta(\alpha) \triangleq \sum_{i=1}^{\infty} i^{-\alpha}=\infty$ for $\alpha=1$. In the two-dimensional case, $\alpha>2$ is required for finite interference since $\sum_{i, k=1}^{\infty}\left(i^{2}+k^{2}\right)^{-1}=\infty$.

\subsection{One-Dimensional Lattices}

\subsubsection{Laplace Transform}

For $\alpha=2$ and $\alpha=4$, the infinite one-sided one-dimensional grid $r_{i}=i$, $i \in \mathbb{N}$, permits a closed-form expression of $\mathcal{L}_{I}(s)$. For $\alpha=2$, following 
[33], we start by expressing the Laplace transform as

$$
\mathcal{L}_{I}(s)=\frac{\prod_{i=1}^{\infty}\left(1+(1-p) s / i^{2}\right)}{\prod_{i=1}^{\infty}\left(1+s / i^{2}\right)}, \quad s \geq 0 .
$$

This permits the application of Euler's product formula

$$
\sin (\pi z) \equiv \pi z \prod_{i=1}^{\infty}\left(1-z^{2} / i^{2}\right), \quad z \in \mathbb{C},
$$

for $z=j \sqrt{(1-p) s}$ (numerator) and $z=j \sqrt{s}$ (denominator). For example, the denominator product is

$$
\prod_{i=1}^{\infty}\left(1+s / i^{2}\right)=\frac{\sin (\pi j \sqrt{s})}{\pi j \sqrt{s}}=\frac{\sinh (\pi \sqrt{s})}{\pi \sqrt{s}}, \quad s \geq 0,
$$

and we obtain

$$
\mathcal{L}_{I}(s)=\frac{1}{\sqrt{1-p}} \cdot \frac{\sinh (\pi \sqrt{s(1-p)})}{\sinh (\pi \sqrt{s})}, \quad s \geq 0 .
$$

From the Bernoulli-l'Hôpital rule it follows that for $p=1$, where all interferers are always active,

$$
\mathcal{L}_{I}(s)=\frac{\pi \sqrt{s}}{\sinh (\pi \sqrt{s})}, \quad s \geq 0 .
$$

While the case $p=1$ may seem artificial since (except for the node at the origin) no nodes in the network are listening, this result will prove useful when analyzing networks with TDMA scheduling.

For $\alpha=4$, we first rewrite (2.3) as

$$
\mathcal{L}_{I}(s)=\frac{\prod_{i=1}^{\infty}\left(1+(1-p) s / i^{4}\right)}{\prod_{i=1}^{\infty}\left(1+s / i^{4}\right)} .
$$

The factorization of both numerator and denominator according to $\left(1-z^{4} / i^{4}\right)=\left(1-z^{2} / i^{2}\right)\left(1+z^{2} / i^{2}\right)$ permits the use of Euler's product formula (2.4) with $z=\sqrt{ \pm j}((1-p) s)^{1 / 4}$ (numerator) and $z=\sqrt{ \pm j} s^{1 / 4}$ (denominator). The two resulting expressions are complex conjugates, and $|\sin (\sqrt{j} x)|^{2}=\cosh ^{2}(x / \sqrt{2})-\cos ^{2}(x / \sqrt{2})$. Hence,

$$
\mathcal{L}_{I}(s)=\frac{\cosh ^{2}\left(\sigma(1-p)^{1 / 4}\right)-\cos ^{2}\left(\sigma(1-p)^{1 / 4}\right)}{\sqrt{1-p}\left(\cosh ^{2} \sigma-\cos ^{2} \sigma\right)},
$$


where $\sigma \triangleq \pi s^{1 / 4} / \sqrt{2}$. For $p=1$, this simplifies to

$$
\mathcal{L}_{I}(s)=\frac{2 \sigma^{2}}{\cosh ^{2} \sigma-\cos ^{2} \sigma}=\frac{\pi^{2} \sqrt{s}}{\cosh ^{2}\left(\frac{\pi s^{1 / 4}}{\sqrt{2}}\right)-\cos ^{2}\left(\frac{\pi s^{1 / 4}}{\sqrt{2}}\right)} .
$$

\subsubsection{Probability Densities}

Next we would like to find an expression for the probability density function (PDF) of $I$. Since a direct calculation does not seem possible, we aim at finding a series expression.

For a finite number of nodes $n$, unit transmit powers, $p=1$ (all nodes transmit), and a general path loss exponent $\alpha, I_{n}$ is an $n$-stage hypoexponential random variable with Laplace transform

$$
\mathcal{L}_{I_{n}}(s)=\prod_{i=1}^{n} \frac{i^{\alpha}}{i^{\alpha}+s},
$$

whose partial fraction expansion is given by

$$
\mathcal{L}_{I_{n}}(s)=\sum_{i=1}^{n} \frac{a_{n, i} i^{\alpha}}{i^{\alpha}+s}, \quad \text { where } a_{n, i} \triangleq \prod_{\substack{k=1 \\ k \neq i}}^{n} \frac{k^{\alpha}}{k^{\alpha}-i^{\alpha}}
$$

By inverse transformation of each term we obtain the PDF

$$
f_{I_{n}}(x)=\sum_{i=1}^{n} a_{n, i} i^{\alpha} \exp \left(-i^{\alpha} x\right), \quad x \geq 0
$$

Since the product-form (2.10) has the product $\prod_{i=1}^{n} i^{\alpha}$ in the numerator (and no term in $s$ ), the residue coefficients $a_{n, i}$ have the properties

that $\sum_{i=1}^{n} a_{n, i}=1, n \in \mathbb{N}$, and $\sum_{i=1}^{n} a_{n, i} i^{\alpha}=0$ for $n>1$. It follows that $f_{I_{n}}(0)=0$ for $n>1$. For $n=1, I_{1}$ follows an exponential distribution, which implies $f_{I_{1}}(0)=1$.

For two special cases, $\alpha=2$ and $\alpha=4$, we can derive the limiting distribution $\lim _{n \rightarrow \infty} f_{I_{n}}(x)$. 
138 Interference in Regular Networks

Special case $\alpha=2$. Using Euler's summation formula again,

$$
\begin{aligned}
\frac{1}{a_{\infty, i}} & =\lim _{x \rightarrow i} \prod_{\substack{k=1 \\
k \neq i}}^{\infty}\left(1-x^{2} / k^{2}\right) \\
& =\lim _{x \rightarrow i} \frac{\sin (\pi x)}{\pi x\left(1-(x / i)^{2}\right)} \\
& =\lim _{x \rightarrow i} \frac{\pi \cos (\pi x)}{\pi\left(1-(x / i)^{2}\right)-\pi x\left(2 x / i^{2}\right)} \\
& =\frac{(-1)^{i+1}}{2} .
\end{aligned}
$$

The PDF of the interference as $n \rightarrow \infty$ is thus

$$
f_{I}(x)= \begin{cases}2 \sum_{i=1}^{\infty}(-1)^{i+1} i^{2} \exp \left(-i^{2} x\right) & \text { if } x>0 \\ 0 & \text { if } x=0 .\end{cases}
$$

Figure 2.1 shows the densities $f_{I_{n}}(x)$ for $n=2,5,12, \infty$. The mean interference in the infinite case is $\mathbb{E}(I)=\sum_{i=1}^{\infty} i^{-2}=\zeta(2)=\pi^{2} / 6$. The corresponding cumulative distribution function (CDF) for finite $n$ is

$$
F_{I_{n}}(x)=\sum_{i=1}^{n} a_{n, i}\left(1-\exp \left(-i^{2} x\right)\right), \quad x \geq 0,
$$

and, for $n \rightarrow \infty$, since $\sum_{i=1}^{n} a_{n, i}=1$ for all $n \in \mathbb{N}$,

$$
F_{I}(x)= \begin{cases}1+2 \sum_{i=1}^{\infty}(-1)^{i} \exp \left(-i^{2} x\right) & \text { if } x>0 \\ 0 & \text { if } x \leq 0 .\end{cases}
$$

Special case $\alpha=4$. Proceeding as for $\alpha=2$,

$$
\begin{aligned}
\frac{1}{a_{\infty, i}} & =\lim _{x \rightarrow i} \prod_{\substack{k=1 \\
k \neq i}}^{\infty}\left(1-x^{4} / k^{4}\right) \\
& =\lim _{x \rightarrow i}\left[\prod_{\substack{k=1 \\
k \neq i}}^{n}\left(1-i^{2} / k^{2}\right) \prod_{\substack{k=1 \\
k \neq i}}^{n}\left(1+i^{2} / k^{2}\right)\right]
\end{aligned}
$$




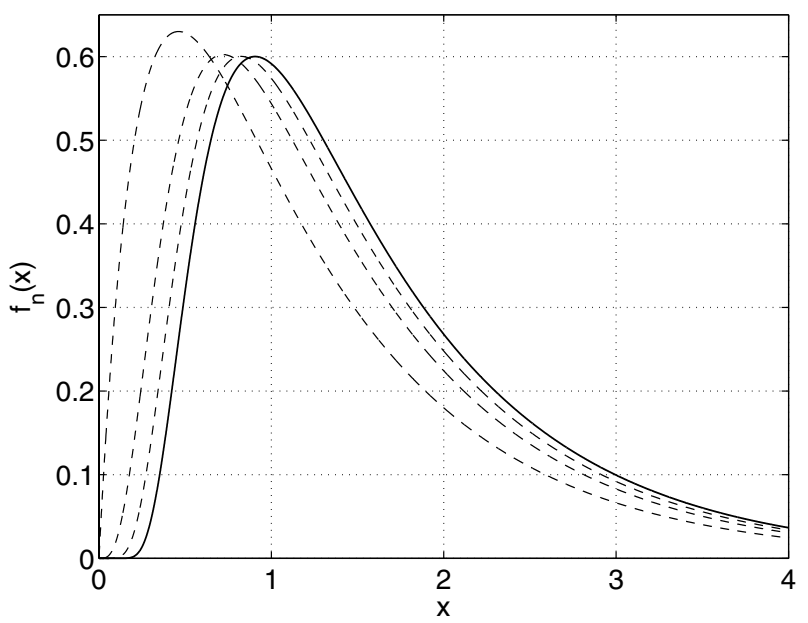

Fig. 2.1 The dashed curves show the probability density $f_{I_{n}}(x)$ from $(2.11)$ for $\alpha=2$ and $n=2$ (left-most curve), $n=5$, and $n=12$, and the solid curve shows the limiting density $f_{I}(x)$ from (2.12).

$$
\begin{aligned}
& =\lim _{x \rightarrow i} \frac{\sin (\pi x) \sinh (\pi x)}{(\pi x)^{2}\left(1-(x / i)^{4}\right)} \\
& =\lim _{x \rightarrow i} \frac{\pi \cos (\pi x) \sinh (\pi x)-\pi \sin (\pi x) \cosh (\pi x)}{2 \pi^{2}\left(1-(x / i)^{4}\right)-\pi^{2} x\left(4 x^{4} / i^{4}\right)} \\
& =\frac{(-1)^{i+1} \sinh (i \pi)}{4 \pi i} .
\end{aligned}
$$

Due to the sinh term, the coefficients $a_{\infty, i}$ decay very quickly, and it is sufficient to consider only the nearest three or even two interferers. Considering only the nearest interferer yields approximately the right tail of the density, but the probabilities of seeing little interference are drastically different. This is essentially a diversity effect.

The probability density for the interference as $n \rightarrow \infty$ is thus

$$
f_{I}(x)= \begin{cases}4 \pi \sum_{i=1}^{\infty} \frac{(-1)^{i+1} i}{\sinh (i \pi)} i^{4} \exp \left(-i^{4} x\right) & \text { if } x>0 \\ 0 & \text { if } x=0 .\end{cases}
$$

Figure 2.2 shows the densities $f_{I_{n}}(x)$ for $n=1,2,3, \infty$. The curve for $n=3$ is virtually indistinguishable from the limiting case. The mean interference in the infinite case is $\mathbb{E}(I)=\sum_{i=1}^{\infty} i^{-4}=\zeta(4)=\pi^{4} / 90$. 


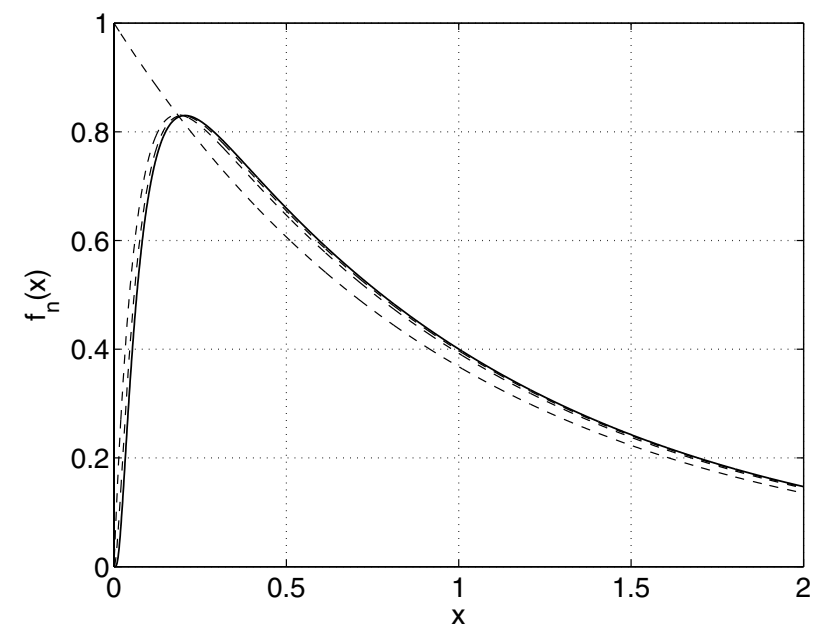

Fig. 2.2 The dashed curves show the probability density $f_{I_{n}}(x)$ from (2.11) for $\alpha=4$ and $n=1,2,3$, and the solid curve shows the limiting density $f_{I}(x)$ from (2.15).

\subsection{Two-Dimensional Lattices}

\subsubsection{Square Lattice}

Consider a network with nodes arranged in the integer lattice without the origin $\mathbb{Z}^{2} \backslash\{o\}$. What is the interference measured at the origin (without fading), or what is the mean interference (with fading)? The lattice sum

$$
I=\sum_{\substack{x \in \mathbb{Z}^{2} \\ x \neq 0}} \ell(\|x\|)
$$

does not have a closed-form expression. However, by grouping the nodes into rings of increasing distances from the origin, we can give good bounds. For example, take the four nearest nodes (distance 1), the four next-nearest (distance $\sqrt{2}$ ), and then (square) rings of $8 k$ nodes for $k=2,3, \ldots$. Each node in ring $k$ is at least at distance $k$ and at most at distance $\sqrt{2} k$, which yields the bounds

$$
4 \ell(1)+4 \ell(\sqrt{2})+\sum_{k=2}^{\infty} 8 k \ell(\sqrt{2} k)<I<4 \ell(1)+4 \ell(\sqrt{2})+\sum_{k=2}^{\infty} 8 k \ell(k) .
$$


For $\ell(r)=r^{-\alpha}$,

$$
\begin{aligned}
& 4\left(1+2^{-\alpha / 2}\right)+8 \cdot 2^{-\alpha / 2}(\zeta(\alpha-1)-1) \\
& \quad<I<4\left(1+2^{-\alpha / 2}\right)+8(\zeta(\alpha-1)-1) .
\end{aligned}
$$

For $\alpha=4$, this is approximately $5.4<I<6.6$. The correct value is right in between, $I=6.037$. A better lower bound is obtained if the average distance to the nodes in ring $k$ is upper bounded by $k(1+\sqrt{2}) / 2$. This is an upper bound since

$$
\frac{k(1+\sqrt{2})}{2} \geq \frac{1}{2}\left(\sqrt{k^{2}+i^{2}}+\sqrt{k^{2}+(k-i)^{2}}\right), \quad \forall 0 \leq i \leq k .
$$

This gives the bound

$$
I \geq 4\left(1+2^{-\alpha / 2}\right)+8 \cdot\left(\frac{1+\sqrt{2}}{2}\right)^{-\alpha}(\zeta(\alpha-1)-1) .
$$

For $\alpha=4$, this is about 5.76. Claiming that the average node distance in ring $k$ is about $k \sqrt{5 / 4}$, obtained from an estimate of $k$ along one axis and $k / 2$ along the other, gives the very good approximation

$$
I \approx 4\left(1+2^{-\alpha / 2}\right)+8 \cdot(5 / 4)^{-\alpha / 2}(\zeta(\alpha-1)-1) .
$$

For $\alpha=4$, this gives $I \approx 5+8(4 / 5)^{2}(\zeta(3)-1)=6.034$, which is very accurate. Figure 2.3 shows the exact value of $I$ as a function of $\alpha$, plus the bounds (2.16) and the approximation (2.18).

To obtain closed-form results (without the zeta function) we note that

$$
\sum_{k=3}^{\infty} k^{-\alpha}=\int_{2}^{\infty}\lceil x\rceil^{-\alpha} \mathrm{d} x<\int_{2}^{\infty} x^{-\alpha} \mathrm{d} x
$$

whereas

$$
\sum_{k=3}^{\infty} k^{-\alpha}=\int_{3}^{\infty}\lfloor x\rfloor^{-\alpha} \mathrm{d} x>\int_{3}^{\infty} x^{-\alpha} \mathrm{d} x
$$

$\lceil x\rceil$ and $\lfloor x\rfloor$ denote the smallest integer larger than or equal to $x$ and the largest integer smaller than or equal to $x$, respectively. It follows that

$$
\zeta(\alpha)<1+2^{-\alpha}+\int_{2}^{\infty} x^{-\alpha} \mathrm{d} x=1+2^{-\alpha} \frac{\alpha+1}{\alpha-1}, \quad \alpha>1,
$$




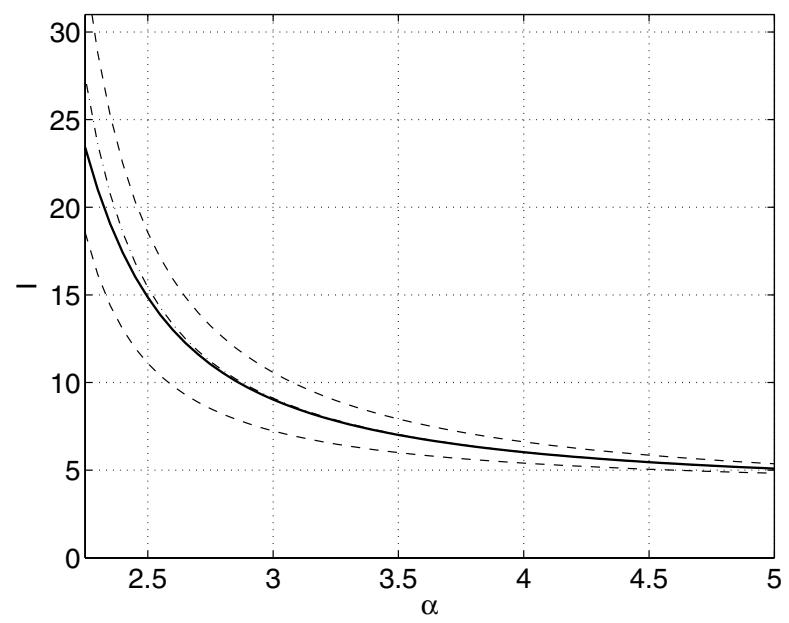

Fig. 2.3 The solid curve is the interference as a function of the path loss exponent $\alpha$. The dashed curves are the bounds (2.16), and the dash-dotted curve is the approximation (2.18), which is very accurate for $\alpha>3$.

which, inserted in (2.16), yields the simple upper bound

$$
I<4\left(1+2^{-\alpha / 2}+4 \cdot 2^{-\alpha} \frac{\alpha}{\alpha-2}\right) .
$$

For $\alpha=4$, this is exactly 7 .

If the integration starts at $5 / 2$ instead of 2 or 3 , a much improved upper bound on the zeta function is obtained:

$$
\zeta(\alpha) \lesssim 1+2^{-\alpha}+\left(\frac{2}{5}\right)^{\alpha-1} \frac{1}{\alpha-1} .
$$

To see that this is indeed an upper bound, let $U_{k}, k=3, \ldots, \infty$, be uniformly randomly distributed over $[k-1 / 2, k+1 / 2]$. It follows from the convexity of $x^{-\alpha}$ and Jensen's inequality that

$$
I=1+2^{-\alpha}+\sum_{k=3}^{\infty} \mathbb{E}\left(U_{k}\right)^{-\alpha}<1+2^{-\alpha}+\sum_{k=3}^{\infty} \mathbb{E}\left(U_{k}^{-\alpha}\right) .
$$

The sum in the upper bound is $\int_{5 / 2}^{\infty} x^{-\alpha} \mathrm{d} x$.

This relatively simple expression upper bounds $\zeta(\alpha)$ very tightly; it is within $0.3 \%$ of the true value for all $\alpha>1$. Plugged into the upper 
bound in (2.16), this yields 6.64 for $\alpha=4$, so indeed it does not appreciably weaken the bound.

In the case of the power law path loss, we may also use results on lattice sums, see, e.g., [55], which typically involve the zeta and other special functions, to express the interference. For the square integer lattice,

$$
I=4 \zeta(\alpha / 2) \beta(\alpha / 2)
$$

where

$$
\beta(x) \triangleq \sum_{i=1}^{\infty} \frac{(-1)^{i+1}}{(2 i-1)^{x}}
$$

is the Dirichlet beta function. $\beta(2)$ is Catalan's constant $K=0.916$. So, for $\alpha=4, I=2 \pi^{2} K / 3 \approx 6.03$. Whereas for other even-dimensional square lattices, similar expressions are known, there are only approximations available for the three-dimensional case.

\subsubsection{Triangular Lattices}

A node deployment in a triangular lattice may make sense for a sensor network. More importantly though, a triangular lattice may be a good model for a CSMA-type network. Assume a high-density network, with $\lambda \gg 1$ nodes per unit area, and a CSMA scheme with carrier sensing radius 1 . Under ideal operation, the transmitting nodes cannot be denser than a triangular grid. Hence the interference in a triangular lattice is an upper bound to the interference in a CSMA network. Again we can partition the interferers to rings of increasing radii; in this case, the rings are hexagons. The six nearest interferers (Ring 1 ) are at distance 1 , the next twelve (Ring 2 ) at distances $\sqrt{3}$ or 2 . Generally, in ring $k$, the distances are at least $\sqrt{3} k / 2$ and at most $k$ (see Figure 2.4), thus the cumulated interference from $m$ rings is

$$
6+\sum_{k=2}^{m} 6 k \cdot k^{-\alpha}<I_{m}<6+\sum_{k=2}^{m} 6 k(\sqrt{3} k / 2)^{-\alpha}, \quad m>1
$$




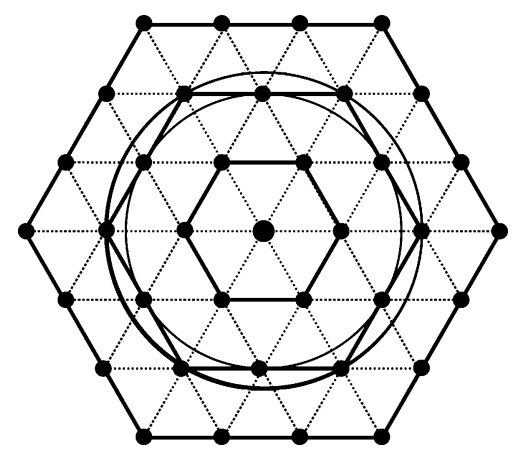

Fig. 2.4 A triangular lattice with three hexagonal rings of nodes. Assuming unit nearestneighbor distances, the two circles indicate that the nodes in the second ring are at distance at least $\sqrt{3}$ and at most 2. Generally, the distance to the nodes in the $k$-th ring is lower bounded by $\sqrt{3} k / 2$ and upper bounded by $k$. Ring $k$ contains $6 k$ nodes.

and, for $m \rightarrow \infty$,

$$
6 \zeta(\alpha-1)<I<6\left(1+\left(\frac{2}{\sqrt{3}}\right)^{\alpha}(\zeta(\alpha-1)-1)\right) .
$$

\subsection{Outage}

As discussed in Section 1.2.2 (p. 133), the outage in Rayleigh fading follows directly from the Laplace transform. So, in all the transforms derived, we obtain the (noise-free) success probability simply by replacing $s$ by the SINR threshold $\theta$. The noise factor is $p_{s}^{W}=$ $\exp (-\theta W /(P \ell(r)))$, where $P$ is the transmit power of the desired transmitter and $r$ is the distance of the link. So the interference factor $p_{s}^{I}$ is much more critical, and for notational simplicity, we henceforth drop the superscript $I$ in $p_{s}^{I}$.

As a sanity check, let us first consider the general case deterministic case $(2.3)$ and let $\theta \rightarrow \infty$. We obtain $\lim _{\theta \rightarrow \infty} p_{s}(\theta)=(1-p)^{n}$, as expected, since a transmission can only succeed if there is no active interferer.

\subsubsection{ALOHA in One-Dimensional Line Networks}

The success probabilities for ALOHA for one-sided line networks follow immediately from the corresponding Laplace transforms. From (2.5) it 
follows that for $\alpha=2$,

$$
p_{s}(\theta)=\frac{1}{\sqrt{1-p}} \cdot \frac{\sinh (\pi \sqrt{\theta(1-p)})}{\sinh (\pi \sqrt{\theta})},
$$

and for $\alpha=4$, it follows from (2.8),

$$
p_{s}(\theta)=\frac{\cosh ^{2}\left(\sigma(1-p)^{1 / 4}\right)-\cos ^{2}\left(\sigma(1-p)^{1 / 4}\right)}{\sqrt{1-p}\left(\cosh ^{2} \sigma-\cos ^{2} \sigma\right)},
$$

where $\sigma \triangleq \pi \theta^{1 / 4} / \sqrt{2}$.

\subsubsection{TDMA in One-Dimensional Line Networks}

Here we assume that interferers are scheduled in an $m$-phase TDMA fashion. In a one-sided line network, this means that nodes $1,1+m, 1+$ $2 m, \ldots$ transmit in slot 1 , nodes $2,2+m, 2+2 m, \ldots$ transmit in slot 2 , and so on, until slot $m$ in which the nodes in $m \mathbb{N}$ transmit. After that, the first set of nodes transmits again. In terms of interference, this means that the network is stretched by a factor $m$, i.e., all the distances are increased by $m$, and the interference from each node is now $\sum_{i=1}^{\infty} \ell(i m)$ instead of just summing over $\ell(i)$. For the power law path loss model, this means all powers are reduced by $m^{\alpha}$. But this is equivalent to reducing the SIR threshold $\theta$ by the same factor! So we can simply take the Laplace transforms for $p=1,(2.6)$ and (2.9), and replace $s$ by $\theta m^{-\alpha}$ instead of by $\theta$ to obtain the success probabilities for TDMA.

For $\alpha=2$, we obtain from (2.6),

$$
p_{s}(\theta)=\frac{\sigma}{\sinh \sigma}, \quad \text { where } \sigma \triangleq \frac{\pi \sqrt{\theta}}{m},
$$

and for $\alpha=4$, from (2.9),

$$
p_{s}(\theta)=\frac{2 \sigma^{2}}{\cosh ^{2} \sigma-\cos ^{2} \sigma}, \quad \text { where } \sigma \triangleq \frac{\pi \theta^{1 / 4}}{\sqrt{2} m} .
$$

Since $\theta$ enters these expressions only through $\theta^{1 / \alpha}$ and $m$-phase TDMA reduces the threshold by $m^{\alpha}$, the exponents for $m$ cancel, and the parameter $\sigma$ is simply inversely proportional to $m$. 


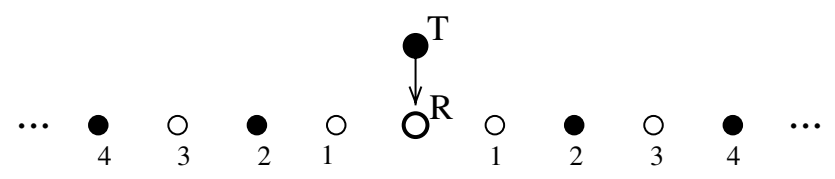

Fig. 2.5 Line network node arrangement for which the success probabilities in the two-sided case are the squares of the success probabilities in the one-dimensional case. The receiver is placed at the origin, and the desired transmitter is at unit distance above. The dark nodes are the ones that are transmitting in the even time slots of a two-phase TDMA channel access scheme.

\subsubsection{Two-Sided Line Networks}

The Laplace transforms and success probabilities we have derived are for one-dimensional line networks. If the distances to the interferers are symmetric, the success probabilities can simply be squared to get the results for the two-sided line networks. For example, if the arrangement is as shown in Figure 2.5, this is the case. 


\section{Interference in Poisson Networks}

In this section we focus on networks whose nodes are distributed as a homogeneous Poisson point process (PPP) (see Section A.1.2). Among all stochastic node distribution models, the PPP and the closely related binomial point process (see Section A.1.1), where a fixed number of nodes is uniformly randomly placed in a certain area, are by far the most popular; they are used in certainly more than $95 \%$, perhaps $99 \%$ of the analytical work on wireless network characterization. The complete spatial randomness or independence property makes the PPP easy to analyze. From the practical side, the PPP model is often justified by claiming that it is suitable when large numbers of nodes are dropped from aircraft (in sensor networks), or when nodes move around independently and uniformly in a certain area.

While interference characterization in large wireless systems is a relatively new topic, a similar type of noise, the so-called shot noise, has been the subject of investigation for more than a century already. We start our discussion by drawing parallels between shot noise and interference. 


\subsection{Shot Noise}

If the nodes in the network are distributed according to a stochastic point process, the interference function stated in (1.1), can be viewed as a random field or, more specifically, as a shot noise process. In one dimension, a basic shot noise process is described as

$$
I(t)=\sum_{i=1}^{\infty} g\left(t-\mathrm{x}_{i}\right)=\sum_{\mathrm{x} \in \Phi} g(t-\mathrm{x}),
$$

where $\Phi=\left\{\mathrm{x}_{i}\right\}_{i=1}^{\infty}$ is a stationary Poisson process on $\mathbb{R}$ and $g(x)$ is the impulse response. Traditionally, shot noise is measured in time, i.e., the points in the PPP are time instants. To model interference in a (onedimensional) wireless network, we replace the time axis by the spatial axis and the impulse response $g(x)$ with the path loss function $\ell(\|x\|)$. This spatial impulse response is two-sided and even, since the wireless signals are assumed to spread equally in both directions. This way, (3.1) becomes the expression for the interference in a one-dimensional network whose (transmitting) nodes are distributed as a Poisson process, with unit transmit powers and no fading.

A generalized shot noise process permits the incorporation of a stochastic impulse response and multi-dimensional point processes:

$$
I(y)=\sum_{x \in \Phi}^{\infty} K_{\times} g(y-\mathbf{x}),
$$

where the coefficients $K_{\mathrm{x}}$ are iid random variables that can be used to model fading. Putting $K g(x)=h \ell(\|x\|)$ shows that (1.1) is a special case of (3.2).

Shot noise processes have been studied at least dating back to Campbell in 1909 [6, 7], who characterized their mean and variance, more fully by Schottky in 1918 [41], while Rice [39] and later Gilbert and Pollak [15] performed extensive investigations on their distribution. Since the path loss law $\ell(\cdot)$ is typically a power law, power law shot noise is most relevant in the context of wireless networks. It was considered by Lowen and Teich in 1990 [32]. In particular, they showed that it does not converge to a normal distribution as the intensity of the point process increases, in contrast to exponentially decaying impulse 
responses. Heinrich and Schmidt studied normal convergence of shot noise processes in detail in [25].

\subsection{Interference Distribution}

In this section we would like to characterize the total interference power measured at the origin, given by

$$
I=I(o) \triangleq \sum_{\mathrm{x} \in \Phi} h_{\mathrm{x}} \ell(\|\mathrm{x}\|),
$$

where $\Phi$ is a point process of interferers on $\mathbb{R}^{d}$. In this case of a uniform (or homogeneous) PPP, it does not matter where the interference is measured; due to Slivnyak's theorem (Theorem A.5) it could even be measured at a point of the process as long as its contribution to $I$ from that point is not considered. In all other cases, however, it does, since the interference seen by a typical point of the point process differs from the interference seen at an arbitrary point of the plane (see Section A.2 on Palm distributions in the appendix).

For channel access, ALOHA is a natural match for the PPP, since ALOHA maintains the distributional properties of the PPP: If all nodes form a PPP of intensity $\lambda$ and transmit independently with probability $p$, the set of transmitters forms a PPP of intensity $p \lambda$. This follows from the (independent) thinning property of the PPP, see Section A.1.2 in the appendix. This implies that if $I(\lambda)$ is the interference in a PPP of intensity $\lambda$ where all nodes transmit, $I(p \lambda)$ is the interference in the same network when ALOHA with probability $p$ is used. Further, due to the superposition property of the PPP, the interference is proportional to $\lambda$, or $p \lambda$.

\subsubsection{Mean Interference}

We start the discussion with a brief derivation of the mean interference.

From Campbell's theorem (Theorem A.2), we have

$$
\begin{aligned}
\mathbb{E}(I)=\sum_{\times \in \Phi} h_{\times} \ell(\|x\|) & =\lambda \mathbb{E}(h) \int_{\mathbb{R}^{d}} \ell(\|x\|) \mathrm{d} x \\
& =\lambda c_{d} d \int_{0}^{\infty} \ell(r) r^{d-1} \mathrm{~d} r
\end{aligned}
$$


where $c_{d}=|b(o, 1)|$ is the volume of the $d$-dimensional unit ball. The fading distribution does not matter, as long as $\mathbb{E}(h)=1$. For $\ell(r)=r^{-\alpha}$,

$$
\mathbb{E}(I)=\lambda c_{d} d \int_{0}^{\infty} r^{d-\alpha-1}=\left.\frac{\lambda c_{d} d}{d-\alpha} r^{d-\alpha}\right|_{0} ^{\infty} .
$$

This diverges for $\alpha<d$ due to the upper integration bound, i.e., the cumulated interference from the large number of distance nodes. For $\alpha>d$, it diverges due to the lower bound, which is a consequence of the path loss law and the property of the PPP that nodes can be arbitrarily close. For $\alpha=d$, both the lower and upper bounds cause problems.

For finite networks, $\alpha<d$ guarantees a finite mean, but in the infinite case it results in infinite interference a.s. On the other hand, for $\alpha>d$, the interference is finite a.s. even for infinite networks since the Laplace transform is non-degenerate, as we will show.

In practice, the path loss is bounded, which can be modeled by, e.g., $\ell(r)=\min \left(1, r^{-\alpha}\right)$. In this case, the mean exists for $\alpha>d$. For finite two-dimensional networks of radius $\rho>1$,

$$
\mathbb{E}\left(I_{\rho}\right)=\lambda\left(\pi+\frac{2 \pi}{\alpha-2}\left(1-\rho^{2-\alpha}\right)\right)
$$

This simple calculation gives some guideline on how large to choose the network area in a simulation, where the behavior of an infinite network is to be explored. If the mean interference in the simulation area (outside radius 1) should match the theoretical mean in an infinite network up to a factor $1-\epsilon$, we have

$$
1-\rho^{2-\alpha}>1-\epsilon \Longrightarrow \rho>\epsilon^{-1 /(\alpha-2)}
$$

For example, if $\epsilon=10^{-3}$ and $\alpha=2.5$, the radius has to be at least $10^{6}$. For values of $\alpha$ smaller than 2.1, the network can hardly be simulated exactly. On the other hand, for $\alpha=4$, a radius of only 32 is sufficient to get within $0.1 \%$. More details on the complexity of simulating PPPs can be found in [50]. The paper also describes how to add a correction term to the interference if the simulation area cannot be chosen large enough to yield the desired accuracy. 


\subsubsection{Interference Distribution Without Fading}

In this subsection we focus on the case of two-dimensional networks and assume there is no fading, i.e., $h_{\mathrm{x}} \equiv 1$ in (3.3), and our goal is to find the characteristic function of the interference and from there, if possible, the distribution.

We follow a basic yet powerful technique as it was used, for example, in [43]. It consists of two steps:

1. Consider first a finite network, say on a disk of radius $a$ centered at the origin, and condition on having a fixed number of nodes in this finite area. The nodes' locations are then iid.

2. Then de-condition on the (Poisson) number of nodes and let the disk radius go to infinity.

Step 1. Consider the interference from the nodes located within distance $a$ of the origin:

$$
I_{a}=\sum_{\mathrm{x} \in \Phi \cap b(o, a)} \ell(\|\mathrm{x}\|) .
$$

For the path loss law $\ell(x)$, it is assumed that it is strictly monotonically decreasing (invertible), and that $\lim _{x \rightarrow \infty} \ell(x)=0$. In the limit $a \rightarrow \infty$, $I_{a}=I$. Let $\mathcal{F}_{I_{a}}$ be the characteristic function (Fourier transform) of $I_{a}$, i.e.,

$$
\mathcal{F}_{I_{a}}(\omega) \triangleq \mathbb{E}\left(e^{j \omega I_{a}}\right) .
$$

Conditioning on having $k$ nodes in the disk of radius $a$,

$$
\mathcal{F}_{I_{a}}(\omega)=\mathbb{E}\left(\mathbb{E}\left(e^{j \omega I_{a}} \mid \Phi(b(o, a))=k\right)\right) .
$$

Given that there are $k$ points in $b(o, a)$, these points are iid uniformly distributed on the disk with radial density

$$
f_{R}(r)= \begin{cases}\frac{2 r}{a^{2}} & \text { if } 0 \leq r \leq a \\ 0 & \text { otherwise }\end{cases}
$$

and the characteristic function is the product of the $k$ individual characteristic functions:

$$
\mathbb{E}\left(e^{j \omega I_{a}} \mid \Phi(b(o, a))=k\right)=\left(\int_{0}^{a} \frac{2 r}{a^{2}} \exp (j \omega \ell(r)) \mathrm{d} r\right)^{k} .
$$


Step 2. The probability of finding $k$ nodes in $b(o, a)$ is given by the Poisson distribution, hence:

$$
\mathcal{F}_{I_{a}}(\omega)=\sum_{k=0}^{\infty} \frac{\exp \left(-\lambda \pi a^{2}\right)\left(\lambda \pi a^{2}\right)^{k}}{k !} \mathbb{E}\left(e^{j \omega I_{a}} \mid \Phi(b(o, a))=k\right) .
$$

Inserting (3.10), summing over $k$, and interpreting the sum as the Taylor expansion of the exponential function, we obtain

$$
\mathcal{F}_{I_{a}}(\omega)=\exp \left(\lambda \pi a^{2}\left(-1+\int_{0}^{a} \frac{2 r}{a^{2}} \exp (j \omega \ell(r)) \mathrm{d} r\right)\right) .
$$

Integration by parts, substituting $r \rightarrow \ell^{-1}(x)$, where $\ell^{-1}$ is the inverse of $\ell$, and letting $a \rightarrow \infty$ yields

$$
\lim _{a \rightarrow \infty} a^{2}\left(-1+\int_{0}^{a} \frac{2 r}{a^{2}} \exp (j \omega \ell(r)) \mathrm{d} r\right)=\int_{0}^{\infty}\left(\ell^{-1}(x)\right)^{2} j \omega e^{j \omega x} \mathrm{~d} x,
$$

so that

$$
\mathcal{F}_{I}(\omega)=\exp \left(j \lambda \pi \omega \int_{0}^{\infty}\left(\ell^{-1}(x)\right)^{2} e^{j \omega x} \mathrm{~d} x\right) .
$$

To get more concrete results, we need to specify the path loss law. For the standard power law $\ell(r)=r^{-\alpha}$, we obtain

$$
\mathcal{F}_{I}(\omega)=\exp \left(j \lambda \pi \omega \int_{0}^{\infty} x^{-2 / \alpha} e^{j \omega x} \mathrm{~d} x\right) .
$$

For $\alpha \leq 2$, the integral diverges, indicating that the interference is infinite almost surely. For $\alpha>2$,

$$
\mathcal{F}_{I}(\omega)=\exp \left(-\lambda \pi \Gamma(1-2 / \alpha) \omega^{2 / \alpha} e^{-j \pi / \alpha}\right), \quad \omega \geq 0 .
$$

The values for negative $\omega$ are determined by the symmetry condition $\mathcal{F}_{I}^{*}(-\omega)=\mathcal{F}_{I}(\omega)$. For $\alpha=4$,

$$
\mathcal{F}_{I}(\omega)=\exp \left(-\lambda \pi^{3 / 2} \exp (-j \pi / 4) \sqrt{\omega}\right) .
$$

This case is of particular interest, since it is the only one where a closed-form expression for the density exists:

$$
f_{I}(x)=\frac{\pi \lambda}{2 x^{3 / 2}} \exp \left(-\frac{\pi^{3} \lambda^{2}}{4 x}\right) .
$$


This is the so-called Lévy distribution, which can also be viewed as an inverse gamma distribution, or as the inverse Gaussian distribution with infinite mean. For other values of $\alpha$, the densities may be expressed in an infinite series [43, Equation (22)].

The characteristic function (3.15) indicates that the interference distribution is a stable distribution with characteristic exponent $2 / \alpha<1$, drift 0 , skew parameter $\beta=1$, and dispersion $\lambda \pi \Gamma(1-2 / \alpha) \cos (\pi / \alpha)$. See Section A.3 for an introduction to stable random variables, in particular (A.14); many more details are given in [40]. The corresponding Laplace transform is (see (A.15))

$$
\mathcal{L}_{I}(s)=\exp \left(-\lambda \pi \Gamma(1-2 / \alpha) s^{2 / \alpha}\right) .
$$

Stable distributions with characteristic exponents less than one do not have any finite moments. In particular, the mean interference diverges, which is due to the singularity of the path loss law at the origin. This also follows immediately from the fact that $\mathbb{E}(I)=$

$-\left.\frac{\mathrm{d}}{\mathrm{d} s} \log \left(\mathcal{L}_{I}(s)\right)\right|_{s=0}=\lim _{s \rightarrow \infty} c s^{2 / \alpha-1}=\infty$. In fact, even when only the nearest interferer, at distance $R_{1}$, is considered, the mean $\mathbb{E}(I)$ does not exist: For $\alpha \geq 2$,

$$
\mathbb{E}\left(I_{1}\right)=\mathbb{E}\left(R_{1}^{-\alpha}\right)=\int_{0}^{\infty} 2 \pi \lambda x^{1-\alpha} \exp \left(-\lambda x^{2}\right) \mathrm{d} x=\infty .
$$

The method of conditioning on a fixed number of nodes, using the iid property of the node locations, and de-conditioning with respect to the Poisson distribution is applicable to many other problems.

\subsubsection{Interference Distribution with Fading}

Here we derive the interference as given in (3.3) for Rayleigh fading. We pursue two separate approaches.

De-conditioning on deterministic network. A first approach, which has been used in [33], is to use the Laplace transform for general deterministic networks (2.3) and de-condition on the distances of the nodes from the origin. In the one-dimensional case, the $n$ smallest 


\section{Interference in Poisson Networks}

distances from the origin $0 \leq R_{1} \leq R_{2} \leq \ldots \leq R_{n}$ are governed by the joint distribution

$$
f_{\left(R_{1}, \ldots, R_{n}\right)}\left(r_{1}, \ldots, r_{n}\right)=(2 \lambda)^{n} \exp \left(-2 \lambda r_{n}\right) \mathbf{1}_{K}\left(r_{1}, \ldots, r_{n}\right),
$$

where $K=\left\{\left(r_{1}, \ldots, r_{n}\right) \mid 0 \leq r_{1} \leq r_{2} \leq \ldots \leq r_{n}\right\}$ is the order cone or hyperoctant in $\mathbb{R}^{n}$, and $\mathbf{1}_{K}$ is the indicator function (see, e.g., [20, Corollary 2]). The factor two stems from the fact that the network extends over the whole real line $\mathbb{R}$, so the point process of distances from the origin $\left\{\left|x_{i}\right|\right\}$ has density two.

For $\alpha=2,(2.3)$ is now the conditional Laplace transform

$$
\begin{aligned}
\mathcal{L}_{I_{n}}^{\mathrm{c}}(s) & =\mathbb{E}\left(\exp \left(-s I_{n}\right) \mid R_{1}=r_{1}, \ldots, R_{n}=r_{n}\right) \\
& =\prod_{i=1}^{n} \frac{r_{i}^{2}}{r_{i}^{2}+s}, \quad s \geq 0 .
\end{aligned}
$$

The superscript $c$ indicates conditioning. Integrating with respect to the joint density yields the de-conditioned Laplace transform

$$
\begin{aligned}
\mathcal{L}_{I_{n}}(s) & =\int_{K} \lambda^{n} \exp \left(-2 \lambda r_{n}\right) \prod_{i=1}^{n} \frac{r_{i}^{2}}{r_{i}^{2}+s} \mathrm{~d} r_{1} \cdots \mathrm{d} r_{n} \\
& =\frac{(2 \lambda)^{n+1}}{n !} \int_{0}^{\infty} \exp (-2 \lambda r)(r-\sqrt{s} \arctan (r / \sqrt{s}))^{n} \mathrm{~d} r
\end{aligned}
$$

where the second line is obtained using induction and partial integration [33]. For all $n \in \mathbb{N}, \mathcal{L}_{I_{n}}(0)=1$, and it can be shown that the limit $\lim _{n \rightarrow \infty} \mathcal{L}_{I_{n}}(s)$ exists for all $s$. So, by continuity, the distribution of the interference $I_{\infty}$ is not defective. Similarly, it can be shown that in the two-dimensional case with $\alpha=2$, the interference distribution is defective, i.e., $\mathbb{P}\left(I_{\infty}=\infty\right)=1$.

For $\alpha=4$ in the two-dimensional case, the squared distances from the origin form again a homogeneous PPP, this time of intensity $\lambda \pi$. So, merely by changing $2 \lambda$ to $\pi \lambda$ in (3.19), we obtain the Laplace transform of the interference caused by the first $n$ interferers in two-dimensional networks for $\alpha=4$. Figure 3.1 shows the Laplace transforms for the one- and two-dimensional cases. It can be seen that the transforms converge quickly. 

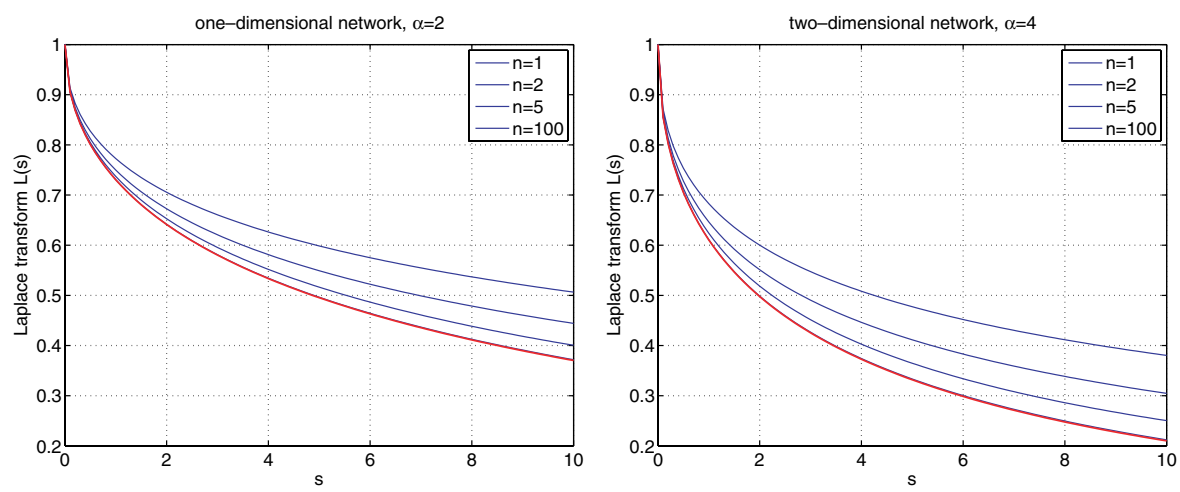

Fig. 3.1 Laplace transform $\mathcal{L}_{I_{n}}(s)$ for the one-dimensional (left) and two-dimensional (right) cases. $n=1,2,5,100$. The curve for $n=1$ is the top curve.

While these are useful results, they are not closed-form; in particular, it does not seem possible to find an explicit expression for the limiting Laplace transform for $n \rightarrow \infty$ from (3.19). Thus we will pursue a different approach to find the Laplace transform of the interference in an infinite network.

Using the probability generating functional. As mentioned in Section 3.1, researchers have found an analogy to shot noise processes to analyze the distributional properties of $I(x)[26,32,39]$.

Here we are using this insight to derive the Laplace transform of the interference. First we map the $d$-dimensional PPP onto $\mathbb{R}^{+}$by letting $\Phi \triangleq\left\{\mathrm{r}_{i}=\left\|\mathrm{x}_{i}\right\|\right\}$ be the distances of the points of a $d$-dimensional uniform PPP of intensity $\lambda$. Per the mapping theorem (Theorem A.1, see also [30]), $\Phi$ is an inhomogeneous PPP with intensity function $\lambda^{\prime}(r)=\lambda c_{d} d r^{d-1}$, where $c_{d}=|b(o, 1)|$ is the volume of the $d$-dimensional unit ball. Considering the interference as a shot noise process (3.1), we can identify the path loss law $\ell(r)=h_{r} r^{-\alpha}$ for iid $h$ with the impulse response of the shot noise process. We would like to calculate the Laplace transform

$$
\mathcal{L}_{I}(s) \triangleq \mathbb{E}\left[e^{-s I}\right]=\mathbb{E}\left[\exp \left(-s \sum_{\mathrm{r} \in \Phi} h_{\mathrm{r}} \mathrm{r}^{-\alpha}\right)\right]
$$


of the interference. The expectation is to be taken over both the point process and the fading. Due to the independence of the fading,

$$
\mathcal{L}_{I}(s)=\mathbb{E}_{\Phi}\left[\prod_{r \in \Phi} \mathbb{E}_{h}\left[\exp \left(-s h_{\mathrm{r}} \mathrm{r}^{-\alpha}\right)\right]\right]
$$

This is a probability generating functional (see Definition A.5) with $v(r)=\mathbb{E} \exp \left(-s h_{r} r^{-\alpha}\right)$, so we have from (A.3)

$$
\mathcal{L}_{I}(s)=\exp \left\{-\mathbb{E}_{h}(\underbrace{\int_{0}^{\infty}\left(1-\exp \left(-s h r^{-\alpha}\right)\right) \lambda^{\prime}(r) \mathrm{d} r}_{A})\right\}
$$

where we flipped the order of integration and expectation. First we calculate the integral:

$$
\begin{aligned}
A & =\lambda c_{d} \int_{0}^{\infty}\left(1-\exp \left(-s h r^{-\alpha}\right)\right) d r^{d-1} \mathrm{~d} r \\
& \left.=\lambda c_{d} \int_{0}^{\infty}\left(1-\exp \left(-s h r^{-1 / \delta}\right)\right) \mathrm{d} r \quad \text { (subst. } r \leftarrow r^{d}\right) \\
& \left.=\lambda c_{d} \int_{0}^{\infty}(1-\exp (-s h / x)) \delta x^{\delta-1} \mathrm{~d} x \quad \text { (subst. } x \leftarrow r^{1 / \delta}\right),
\end{aligned}
$$

where $\delta \triangleq d / \alpha$. To calculate this integral, we note that it is the expected value

$$
\mathbb{E}\left[\left((X / s h)^{-1}\right)^{\delta}\right]
$$

of an exponential random variable $X$ with mean 1 . Since $\mathbb{E}\left(X^{p}\right)=$ $\Gamma(1+p)$ by the definition of the gamma function,

$$
\Gamma(p) \triangleq \int_{0}^{\infty} t^{p-1} e^{-t} \mathrm{~d} t
$$

it follows that

$$
\mathbb{E}\left[\left((X / s h)^{-1}\right)^{\delta}\right]=(s h)^{\delta} \Gamma(1-\delta) .
$$

So, with $A=\lambda c_{d}(h s)^{\delta} \Gamma(1-\delta)$, we obtain

$$
\mathcal{L}_{I}(s)=\exp \left(-\lambda c_{d} \mathbb{E}\left[h^{\delta}\right] \Gamma(1-\delta) s^{\delta}\right) .
$$


The only difference to (3.18) is the additional term $\mathbb{E}\left(h^{\delta}\right)$, which accounts for the fading. For non-unit transmit powers $P_{t}, s$ is simply replaced by $P_{t} s$, i.e., the transmit powers enters the Laplace transform through an additional factor $P_{t}^{\delta}$ in the exponential. Note that $(3.20)$ is only valid for $\delta<1$. So:

- For $\alpha \leq d$, we have $I=\infty$ a.s. This is a consequence of the cumulated interference from the many far transmitters whose signal powers do not decay fast enough to keep the interference power finite. For a finite network, the interference would be finite.

- For $\alpha>d$ we have $I<\infty$ a.s. but $\mathbb{E}(I)=\infty$ due to the singularity of the path loss law at the origin. Even if we consider only the nearest interferer, $\mathbb{E}(I)$ is infinite. If a bounded path loss law is used, all moments exist.

In the case of Rayleigh fading, $\mathbb{E}\left(h^{\delta}\right)=\Gamma(1+\delta)$, using the properties of the gamma function, we obtain the closed-form result

$$
\mathcal{L}_{I}(s)=\exp \left(-\lambda c_{d} s^{\delta} \frac{\pi \delta}{\sin (\pi \delta)}\right)
$$

As in the non-fading case, the interference has a stable distribution with characteristic exponent $\delta$, drift 0 , and skew parameter $\beta=1$; the dispersion here is $\lambda c_{d} \mathbb{E}\left(h^{\delta}\right) \Gamma(1-\delta) \cos (\delta \pi / 2)$, see Section A.3.

As shown in (3.17), for $\delta=1 / 2$, the PDF and CDF exist. With $\Gamma(3 / 2)=\sqrt{\pi} / 2$, the Lévy PDF is in the two-dimensional case $(\alpha=4)$

$$
f_{I}(x)=\frac{\lambda}{4}\left(\frac{\pi}{x}\right)^{3 / 2} \exp \left(-\frac{\pi^{4} \lambda^{2}}{16 x}\right)
$$

and the $\mathrm{CDF}$ is

$$
F_{I}(x)=1-\operatorname{erf}\left(\frac{\pi^{2} \lambda}{4 \sqrt{x}}\right)
$$

where $\operatorname{erf}(x)=2 \int_{0}^{x} \exp \left(-t^{2}\right) \mathrm{d} t / \sqrt{\pi}$ is the standard error function. 
For general $\delta$, the probability density of the interference can be expressed as [32]

$$
f_{I}(x)=\frac{1}{\pi x} \sum_{i=1}^{\infty} \frac{(-1)^{i+1} \Gamma(1+i \delta) \sin (\pi i \delta)}{i !} \cdot\left(\frac{\lambda c_{d} \Gamma(1-\delta) \mathbb{E}\left(h^{\delta}\right)}{x^{\delta}}\right)^{i} .
$$

From this series it is apparent that as $x \rightarrow \infty$, the term for $i=1$ becomes dominant, and

$$
\begin{aligned}
f_{I}(x) & \sim \frac{1}{\pi x^{\delta+1}} \lambda c_{d} \underbrace{\Gamma(1+\delta) \Gamma(1-\delta) \sin (\pi \delta)}_{\pi \delta} \mathbb{E}\left(h^{\delta}\right) \\
& \sim \lambda c_{d} \delta \mathbb{E}\left(h^{\delta}\right) x^{-(1+\delta)}, \quad x \rightarrow \infty .
\end{aligned}
$$

In the non-fading case, we may use the distribution of the distances to the $n$-th nearest neighbor to generalize this result to the behavior of the tail probability for the $n$-th interferer. The CCDF of the distance to the $n$-th nearest neighbor $R_{n}$ is [19]

$$
\mathbb{P}\left(R_{n}>r\right)=\sum_{k=0}^{n-1} \frac{\left(\lambda c_{d} r^{d}\right)^{k}}{k !} \exp \left(-\lambda c_{d} r^{d}\right)=\frac{\Gamma\left(n, \lambda c_{d} r^{d}\right)}{\Gamma(n)},
$$

where $\Gamma(\cdot, \cdot)$ is the upper incomplete gamma function. So, with $I_{n}=$ $R_{n}^{-\alpha}$,

$$
\mathbb{P}\left(I_{n}<x\right)=\sum_{k=0}^{n-1} \frac{\left(\lambda c_{d} x^{-\delta}\right)^{k}}{k !} \exp \left(-\lambda c_{d} x^{-\delta}\right)=\frac{\Gamma\left(n, \lambda c_{d} x^{-\delta}\right)}{\Gamma(n)} .
$$

For the tail probability we need the CCDF, obtained by summing from $n$ to $\infty$ instead of 0 to $n-1$. For $x \rightarrow \infty$, the dominant term will be the one for $k=n$. Since $\exp \left(-x^{-\delta}\right) \sim 1-x^{-\delta}$,

$$
\begin{aligned}
\mathbb{P}\left(I_{n}>x\right) & \sim\left(1-\lambda c_{d} x^{-\delta}\right) \frac{\left(\lambda c_{d} x^{-\delta}\right)^{n}}{n !} \\
& \sim \frac{1}{n !}\left(\lambda c_{d}\right)^{n} x^{-n \delta}, \quad x \rightarrow \infty .
\end{aligned}
$$

Plugging in $n=1$ and taking the derivative, it is confirmed that this is consistent with (3.25).

These results on the tail probabilities imply that $\mathbb{E}\left(I_{n}^{p}\right)$ exists for $p<n \delta$. For example, if interference-canceling techniques are used and 
the interference from the $k$ nearest interferers can be canceled, we need $k>\alpha$ in two-dimensional networks to have a finite second moment.

With fading, we can infer from $(3.24)$ that a factor $\left(\mathbb{E}\left(h^{\delta}\right)\right)^{n}$ has to be added.

The fact that the fading distribution only enters the Laplace transform (3.20) through its $\delta$-th moment may appear surprising at first. It is, however, an instance of a result by Gilbert and Pollak [15] (see also [32]) who have shown that in the one-dimensional case, an ensemble of stochastic response functions, in our case $h x^{-\alpha}$, has an equivalent deterministic impulse response function $\ell_{\mathrm{eq}}(x)=c x^{-\alpha}$ satisfying

$$
\mathbb{E}_{h}\left|\left\{x: h x^{-\alpha}>y\right\}\right|=\left|\left\{x: c x^{-\alpha}>y\right\}\right| \quad \forall y .
$$

To find $c$, we note that the LHS is $\mathbb{E}\left(h^{1 / \alpha}\right) x^{-1 / \alpha}$, and the RHS is $c^{1 / \alpha} x^{-1 / \alpha}$. So $c=\left(\mathbb{E}\left(h^{1 / \alpha}\right)\right)^{\alpha}$. So, replacing $\ell(r)=r^{-\alpha}$ by $\ell_{\text {eq }}(r)=$ $\left(\mathbb{E}\left(h^{1 / \alpha}\right)\right)^{\alpha} r^{-\alpha}$ gives the correct first-order statistics for the interference with fading process $h$ in the one-dimensional case. In $d$ dimensions, the LHS is $c_{d} \mathbb{E}\left(h^{\delta}\right) x^{-\delta}$, and the RHS is $c_{d} c^{\delta} x^{-\delta}$, and the equivalent deterministic path loss law is

$$
\ell_{\mathrm{eq}}(r)=\left(\mathbb{E}\left(h^{\delta}\right)\right)^{1 / \delta} r^{-\alpha} .
$$

As an example, plugging in this deterministic path loss law in (3.14) yields the correct expression for the Laplace transform for the fading case (3.20), since $x^{-\delta}$ in (3.14) is replaced by $(x / c)^{-\delta}$, which produces the factor $\mathbb{E}\left(h^{\delta}\right)$ as desired. The equivalence only holds up to the mean, but since these distributions do not have any finite moments, the Laplace transforms for the cases with stochastic fading and with the equivalent deterministic path loss law are identical. As we can see from the series expression of the probability density (3.24), the complete density cannot be derived using the equivalent path loss law.

In [26] the amplitude distribution of the interference was studied; they show that if each interfering signal is spherically symmetric, the interference amplitude has a symmetric Lévy-stable distribution (skew 0) with characteristic exponent $4 / \alpha$. This is consistent with our result for the interference power, since the amplitude decays with distance to the power $\alpha / 2$. They also analyzed the convergence properties 
to stable distribution as the number of nodes increases, and they considered lognormal fading (shadowing).

\subsection{SIR Distribution and Outage}

As mentioned in the introduction, the Laplace transform is exactly the distribution of the SIR if the power from the desired transmitters $S$ is exponentially distributed (Rayleigh fading). So while closed-form expressions for the interference itself do not exist, they are available in certain cases for the SIR.

For a transmitter-receiver distance $r$, the received signal power $S$ is exponential with mean $r^{-\alpha}$. The success probability $p_{s}(\theta)=\mathbb{P}(S>$ $I \theta)=\mathbb{E} \exp \left(-I \theta r^{\alpha}\right)$ is the Laplace transform of the interference evaluated at $s=\theta r^{\alpha}$. So, in a $d$-dimensional interference-limited networks whose nodes are distributed as a uniform PPP of intensity $\lambda$ with ALOHA channel access with probability $p$, the outage probability for Rayleigh fading desired signal strength $S$ follows from (3.20), replacing $s$ by $\theta r^{\alpha}$ :

$$
p_{s}(\theta)=\exp \left(-p \lambda c_{d} r^{d} \mathbb{E}\left(h^{\delta}\right) \Gamma(1-\delta) \theta^{\delta}\right)
$$

Here we have used the fact that ALOHA channel access performs independent thinning of the PPP, which results in a PPP of lower intensity. While $S$ needs to be Rayleigh, the interferers' channels may be subject to a different type of fading (or no fading), all that matters is $\mathbb{E}\left(h^{\delta}\right)$. For Rayleigh fading interferers, we obtain from (3.21)

$$
p_{s}(\theta)=\exp \left(-p \lambda c_{d} r^{d} \frac{\pi \delta}{\sin (\pi \delta)} \theta^{\delta}\right) .
$$

This result has been derived in $[3,54]$.

Since the nearest-neighbor distance scales as $\lambda^{-1 / d}$, this shows that nearest-neighbor communication is always possible with constant success probability, irrespective of the network density.

If the received signal strength from the desired transmitter is not Rayleigh fading, there are no known closed-form expressions for the outage. If the fading random variables have a square integrable density, an integral expression for the success probability is given 
in $[4$, Proposition 2.2]. Bounds on the outage probability have been derived in $[49,51]$ in the context of the so-called transmission capacity, defined to be the maximum transmitter density given an outage constraint for transmissions to a receiver at fixed distance.

\subsection{Extremal Behavior}

When analyzing the outage probabilities of nearest-neighbor transmission or the benefits of interference cancellation, it is necessary to relate the power from the strongest interferer to the total interference. This strongest "interferer" may be the desired transmitter or the one to be canceled. Here we derive the extreme value statistics for the maximum interference in two-dimensional networks. As in Section 3.2.2, we start by putting $k$ nodes uniformly iid on a disk of radius $a$. For the power path loss law, without fading, the power at the origin from each node, $R^{-\alpha}$, is distributed as

$$
\mathbb{P}\left(R^{-\alpha}<x\right)=1-\frac{1}{a^{2}} x^{-2 / \alpha}, \quad x \geq a^{-\alpha} .
$$

Let $\delta \triangleq 2 / \alpha$. Let $M_{k}$ be the maximum of the $k$ interference powers and $F_{M_{k}}(x)$ its CDF:

$$
F_{M_{k}}(x)=\left(1-\frac{1}{a^{2}} x^{-\delta}\right)^{k}, \quad x \geq a^{-\alpha} .
$$

Since the CCDF decays like $x^{-\delta}$, the asymptotic extremal distribution $F_{M_{\infty}}$ is a Fréchet distribution, which has the shape $\exp \left(-x^{-\delta}\right)$, up to a possible shift and dilatation (see, e.g., [18, Theorem 6.2]). This means that there are sequences of shift parameters $a_{k}$ and dilatation parameters $b_{k}$, such that

$$
\lim _{k \rightarrow \infty} F_{M_{k}}\left(a_{k}+b_{k} x\right)=\exp \left(-x^{-\delta}\right) .
$$

The standard Fréchet distribution on the right side is the limit $\lim _{k \rightarrow \infty}\left(1-x^{-\delta} / k\right)^{k}$. So $a_{k}$ and $b_{k}$ are given by the identity

$$
F_{M_{k}}\left(a_{k}+b_{k} x\right) \equiv\left(1-\frac{x^{-\delta}}{k}\right)^{k},
$$


from which we find $a_{k} \equiv 0, b_{k}=\left(k / a^{2}\right)^{1 / \delta}$. Hence

$$
F_{M_{k}}(x) \sim \exp \left(-\left(k / a^{2}\right) x^{-\delta}\right) \quad \text { as } k \rightarrow \infty .
$$

If we let $k$ and $a^{2}$ go to infinity such that the density $\lambda=k /\left(\pi a^{2}\right)$ stays constant, we have

$$
F_{M_{\infty}}(x)=\exp \left(-\lambda \pi x^{-\delta}\right)
$$

In this limiting case, the nodes form a PPP on $\mathbb{R}^{2}$, so we should be able to get the same result by simply calculating the interference $R_{1}^{-\alpha}$ from the nearest interferer, assumed at distance $R_{1}$. Indeed, $\mathbb{P}\left(R_{1}<\right.$ $r)=1-\exp \left(-\lambda \pi r^{2}\right)$, from which (3.31) follows immediately.

Rewriting the CDF in (3.30) in terms of the rescaled variables $x k^{-1 / \delta}$ such that the right side becomes independent of $k$ shows that $M_{k}=\Theta\left(k^{1 / \delta}\right)$. Since $\delta<1, k^{1 / \delta} \gg k$ for large $k$, so the sum is not proportional to $k$, which is consistent with the fact that the mean diverges. We have found in Section 3.2 that the distribution of the sum of all interferers is a Lévy-stable distribution with the same power-law tail as the Fréchet distribution, which shows that the maximum and the sum are of the same order. This implies, in turn, that if the power from the nearest transmitter is the desired signal, the SIR converges to a non-zero constant as $k \rightarrow \infty$. This fact has been exploited in, for example, in [16] to derive the capacity scaling law for ad hoc networks with mobile nodes. It hinges, however, critically on the homogeneity of the path loss law.

The situation is the same in the presence of fading — as long as the expectation of the fading random variable is finite, which is generally the case. Then the heavy tail of the interference distribution can only be due to the singularity of the path loss law.

\subsection{Power Control}

We introduce the notion of perceived transmit power $P$, which is the transmit power multiplied by the fading coefficient. Previously, we did not consider power control but fading, so we had $P=h$. With neither fading nor power control, $P \equiv 1$. With power control and no fading, $P$ is just the transmit power $P_{t}$, and with power control and fading, 
$P=h P_{t}$. We assume that the power control schemes will result in the perceived transmit powers $P_{i}$ to be iid across the transmitters, which allows us to use the result (3.20) from the previous section since for the interference it does not matter if the randomness is due to fading or power control, or the combination of both. The main change is to replace $\mathbb{E}\left(h^{\delta}\right)$ by $\mathbb{E}\left(P^{\delta}\right)$; hence our task in this section is mainly the determination of this $\delta$-th moment.

We focus on pairwise power control between a transmitter and its receiver; in particular, we consider the case where each node transmits to its nearest neighbor, assumed at distance $R_{1}$. As it would not make sense to have all nodes in the network transmit, we introduce an ALOHA parameter $p$ to obtain a thinned PPP of intensity $\lambda p$ of transmitters. This changes (3.20) slightly to

$$
\mathcal{L}_{I}(s)=\exp \left(-p \lambda c_{d} \mathbb{E}\left(P^{\delta}\right) \Gamma(1-\delta) s^{\delta}\right) .
$$

\subsubsection{Channel Inversion Without Fading}

In this case, nodes compensate for the large-scale path loss by channel inversion. The transmit power $P_{t}=P=R_{1}^{\alpha}$ is Weibull distributed

$$
\mathbb{P}(P \leq x)=1-\exp \left(-\lambda c_{d} x^{\delta}\right)
$$

with the moments

$$
\mathbb{E}\left(P^{m}\right)=\frac{\Gamma(1+m / \delta)}{\left(\lambda c_{d}\right)^{m / \delta}} .
$$

One might argue that instead of $\lambda, \lambda(1-p)$ should be used as the relevant density for the nearest-neighbor distance since this is the density of the non-transmitting nodes. This would require a simple change in the network density. However, ALOHA is an uncoordinated MAC scheme, so it is not possible for a transmitter to know when its intended receiver will be available to actually receive. Since $\mathbb{E}\left(P^{\delta}\right)=1 /\left(\lambda c_{d}\right)$ we obtain from (3.20)

$$
\mathcal{L}_{I}(s)=\exp \left(-p \Gamma(1-\delta) s^{\delta}\right),
$$

which is independent of the network density! So, no matter how dense we make the network, if the transmitters talk to their nearest neighbors 
and compensate for the path loss so that their signal arrives with unit power, the interference distribution does not change. This is of course not a coincidence: If nodes are ordered according to their distances $R_{i}$ from a given point, $\left\{R_{i}^{d}\right\}$ forms a homogeneous PPP of intensity $\lambda c_{d}\left[20\right.$, Corollary 2]. So the mean distance to the first node $R_{1}$ is $1 /\left(\lambda c_{d}\right)^{1 / d}$. The transmit power is proportional to $R_{1}^{\alpha}$, so $P^{\delta}=R_{1}^{d}$, which is proportional to $1 /\left(\lambda c_{d}\right)$.

Compared to the case without power control, where we assumed unit transmit power, the mean power here is $\mathbb{E}(P)=\Gamma(1+1 / \delta) /\left(\lambda c_{d}\right)^{1 / \delta}$. If we compensate for the change in mean transmit power, the power distribution is

$$
\mathbb{P}(P \leq x)=1-\exp \left(-(\Gamma(1+1 / \delta) x)^{\delta}\right),
$$

and the Laplace transform takes the form

$$
\mathcal{L}_{I}(s)=\exp \left(-p \lambda c_{d} \Gamma(1+1 / \delta)^{-\delta} \Gamma(1-\delta) s^{\delta}\right) .
$$

Comparing the resulting interference with the interference in Rayleigh fading (without power control), we note that the coefficients $\Gamma(1+\delta)>$ $\Gamma(1+1 / \delta)^{-\delta}$ for $\delta<1$ and that the ratio diverges as $\delta \rightarrow 0$ since $\lim _{\delta \rightarrow 0} \Gamma(1+1 / \delta)^{-\delta}=0$. This indicates that power control causes less interference than Rayleigh fading would, and that the difference increases with increasing path loss exponent $\alpha$ (for a fixed number of dimensions $d$ ).

While the power levels are spatially iid, it cannot be assumed that they are also temporally iid, since the distance to a node's nearest neighbor is unlikely to change from time slot to time slot. The temporal correlation structure depends on the level of mobility and the length of a communication session between two nodes.

\subsubsection{Power Control and Fading}

Compensation for large-scale path loss. If the channel is Rayleigh fading but the transmitters only compensate for the large-scale path loss to the nearest receiver, each interferer's power is the product of the Weibull random variable $R_{1}^{\alpha}$ and an exponential random variable $h, P=R_{1}^{\alpha} h$. Generally, the product of two independent random variables $X$ and $Y$ 
with distributions $F_{X}(x)$ and $F_{Y}(x)$, respectively, is distributed as

$$
F_{X Y}(z)=\mathbb{E}_{Y}\left(F_{X}(z / Y)\right)=\mathbb{E}_{X}\left(F_{Y}(z / X)\right) .
$$

In this case, the product distribution is

$$
F_{P}(x)=\int_{0}^{\infty}(1-\exp (x / r)) \lambda c_{d} \delta r^{\delta-1} \exp \left(-\lambda c_{d} r^{\delta}\right) \mathrm{d} r .
$$

This integral can be expressed using the infinite series [35]

$$
\begin{aligned}
F_{P}(x)= & \left(\lambda c_{d}\right)^{1 / \delta} x \sum_{k=0}^{\infty} \frac{\Gamma\left(1-\frac{1}{\delta}(k+1)\right)}{(k+1) !}\left(-\left(\lambda c_{d}\right)^{1 / \delta} x\right)^{k} \\
& +\lambda c_{d} x^{\delta} \sum_{k=0}^{\infty} \frac{\Gamma(1-\delta(k+1))}{(k+1) !}\left(-\lambda c_{d} x^{\delta}\right)^{k},
\end{aligned}
$$

which exhibits a striking symmetry between the two parts in this expression. This representation is only valid if $1 / \delta \notin \mathbb{N}$ since the gamma function diverges for negative integers.

The moment $\mathbb{E}\left(P^{\delta}\right)$ is easy to find:

$$
\mathbb{E}\left(P^{\delta}\right)=\mathbb{E}\left(\left(R_{1}^{\alpha}\right)^{\delta} h^{\delta}\right)=\mathbb{E}\left(R_{1}^{d}\right) \mathbb{E}\left(h^{\delta}\right)=\frac{1}{\lambda c_{d}} \Gamma(1+\delta) .
$$

With this, we obtain

$$
\mathcal{L}_{I}(s)=\exp \left(-p \Gamma(1+\delta) \Gamma(1-\delta) s^{\delta}\right),
$$

and, for the case where the transmit powers are normalized to 1 ,

$$
\mathcal{L}_{I}(s)=\exp \left(-p \lambda c_{d} \frac{\Gamma(1+\delta)}{\Gamma(1+1 / \delta)^{\delta}} \Gamma(1-\delta) s^{\delta}\right) .
$$

Compensation for path loss and fading. If the transmitters have the complete channel information, including the fading realization, they can compensate for the complete path loss. The iid process governing the interference power is $R_{1}^{\alpha} h_{2} / h_{1}$, where $h_{1}$ is the fading coefficient of the channel to the transmitter's destination and $h_{2}$ is the coefficient of the channel to the point where interference is measured, see Figure 3.2. Let $H \triangleq h_{2} / h_{1}$. In Rayleigh fading,

$$
F_{H}(x)=\frac{x}{x+1} .
$$




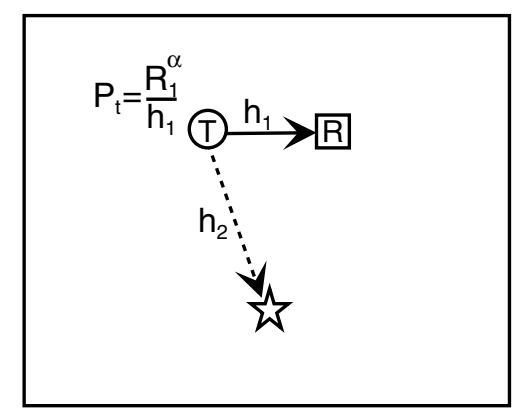

Fig. 3.2 Illustration for the case where power control is used to compensate for large-scale path loss and fading. Interference is measured at the location of the star. The transmitter $T$ transmits at power $P_{t}=R_{1}^{\alpha} / h_{1}$ to compensate for its channel to its receiver R. The channel from $\mathrm{T}$ to the location of the star is subject to fading with coefficient $h_{2}$, so the perceived power is $R_{1}^{\alpha} h_{2} / h_{1}$.

With $P=R^{\alpha} h_{2} / h_{1}$,

$$
F_{P}(x)=\int_{0}^{\infty} \frac{1-\exp \left(-\lambda c_{d}(x / y)^{\delta}\right)}{(y+1)^{2}} \mathrm{~d} y
$$

The $\delta$-moment is of $H$ is

$$
\mathbb{E}\left(H^{\delta}\right)=\mathbb{E}\left(h_{2}^{\delta}\right) \mathbb{E}\left(h_{1}^{-\delta}\right)=\Gamma(1+\delta) \Gamma(1-\delta),
$$

from which

$$
\mathbb{E}\left(P^{\delta}\right)=\frac{\Gamma(1+\delta) \Gamma(1-\delta)}{\lambda c_{d}}
$$

and

$$
\mathcal{L}_{I}(s)=\exp \left(-p \Gamma(1+\delta) \Gamma(1-\delta)^{2} s^{\delta}\right)
$$

follow.

In Figure 3.3, the values of $\mathbb{E}\left(P^{\delta}\right)$ are shown for the different cases. It can be seen that full channel inversion has the most drastic impact on the interference. Note that in this case, normalization by the mean power is not possible, since $\mathbb{E}\left(1 / h_{1}\right)=\infty$.

A detailed discussion of the impact of power control to compensate for path loss and fading can be found in [49]. 


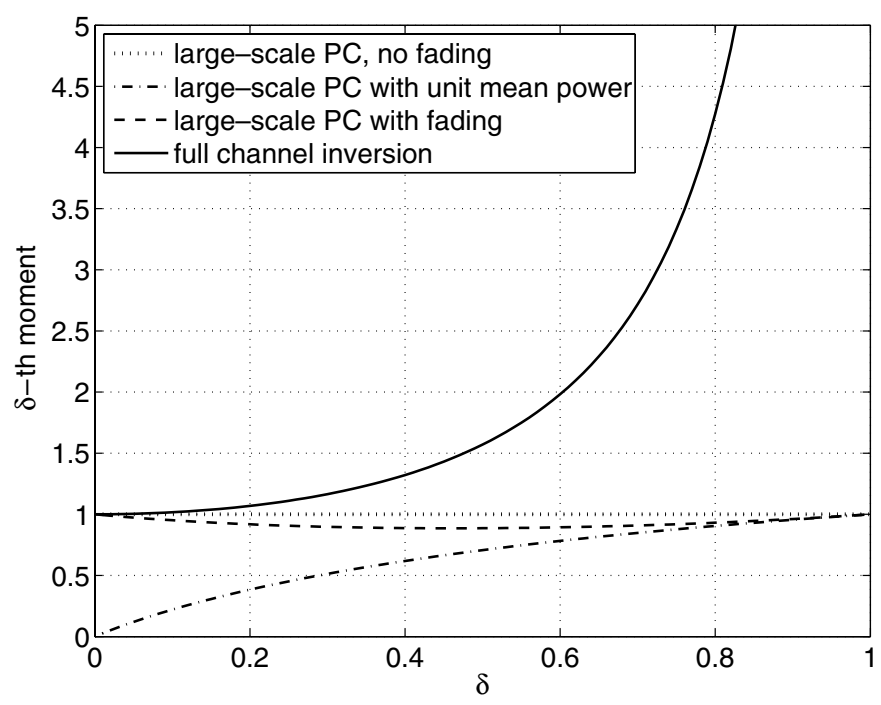

Fig. $3.3 \delta$-th Moment of perceived transmission power $P$ for four cases of power control ((3.32), (3.33), (3.37), (3.40)). The curves are normalized to $\lambda c_{d}=1$.

\subsubsection{Impact on Outage Probabilities}

Until now, we have only studied the effect of power control on the interference, and the conclusion is that power control in networks with fading usually increases the interference. In contrast, power control leads to an improvement of the channel to the intended receiver. These two effects need to be traded off against each other. This trade-off was studied in [28] for the scenario where each transmitter has its intended receiver at a fixed distance, and power control is used to compensate for the fading. They found that fractional power control is optimum, where the transmit power is chosen in proportion to $h^{-1 / 2}$, rather than complete channel inversion, i.e., transmitting at power $h^{-1}$. Fractional power control offers a better trade-off between improving the own link versus causing more interference to the other users. It also has the advantage that the mean transmit power $\mathbb{E}\left(h^{-1 / 2}\right)$ is finite; it is equal to $\sqrt{\pi}$. [51] considered the case where the intended receiver is located uniformly on an annulus.

Here we discuss the effect of large-scale power control on the outage probability in the case of nearest-neighbor communication. Without 
power control and $R$ being the distance to the nearest neighbor, a transmitter succeeds with probability

$$
\begin{aligned}
\bar{p}_{s}(\theta) & =\mathbb{E}_{R}\left(\exp \left(-p \lambda c_{d} R^{d} \Gamma(1+\delta) \Gamma(1-\delta) \theta^{\delta}\right)\right) \\
& =\frac{1}{1+p \Gamma(1+\delta) \Gamma(1-\delta) \theta^{\delta}} .
\end{aligned}
$$

This confirms that irrespective of the network density, each node can transmit to its nearest neighbor with constant success probability. With large-scale power control (inverting the large-scale path loss), each transmitter has the same success probability (from (3.37))

$$
p_{s}^{\mathrm{pc}}(\theta)=\exp \left(-p \Gamma(1+\delta) \Gamma(1-\delta) \theta^{\delta}\right),
$$

which is slightly lower. Essentially, what power control does is to compensate for the distance to the nearest neighbor, which is the same as replacing $R^{d}$ by its expectation. From Jensen's inequality follows that

$$
p_{s}^{\mathrm{pc}}(\theta)=\exp \left(-c \mathbb{E}\left(R^{d}\right)\right)<\bar{p}_{s}=\mathbb{E}\left(\exp \left(-c R^{d}\right)\right), \quad c>0 .
$$

In the low-outage regime, for small values of $p$ or $\theta$, the difference is small; it reaches $3-5 \%$ around success probabilities of $70 \%$, and the gap increases further at higher outage rates. So while power control is slightly harmful from this point of view, it has the big advantage of a fixed success probability. Without power control, there is a significant variance in the success probabilities $p_{s}(\theta, R)$. This variance is (obviously) zero for $p_{s} \uparrow 1$ and $p_{s} \downarrow 0$, and it reaches a maximum of 0.09 when $p \Gamma(1+\delta) \Gamma(1-\delta) \theta^{\delta}$ equals the golden ratio $(1+\sqrt{5}) / 2$. At that value, $\bar{p}_{s} \approx 38 \%$ and $p_{s}^{\mathrm{pc}} \approx 20 \%$. A standard deviation of 0.3 shows that the success rates for many transmitters will be lower than $p_{s}^{\mathrm{pc}}$ if there is no power control.

\subsection{Spread-Spectrum Communication}

With spread-spectrum communications, the effective interference can be reduced by a factor $M$ that is commonly called the processing gain or spreading factor. Two common types of spreading are directsequence spread-spectrum (DS-SS) and frequency-hopping spreadspectrum (FH-SS). Interestingly, as discussed in [1], the effects of these 
techniques on the interference and outage are quite different, although they both require an $M$-fold increase in system bandwidth.

With DS-SS, all transmitting nodes still cause interference, but the interference is scaled by a factor $M$. The outage in Rayleigh fading is affected by a reduction of the SIR threshold $\theta$ by a factor of $M$ since

$$
p_{s}^{\mathrm{DS}}(\theta, M)=\mathbb{E}\left(e^{-\theta I / M}\right)=p_{s}(\theta / M) .
$$

So, from (3.29), we see that

$$
\text { DH-SS: } \quad \frac{\log p_{s}(\theta / M)}{\log p_{s}(\theta)}=M^{-\delta} .
$$

On the other hand, with FH-SS, the density of interferers is reduced by a factor of $M$, which implies

$$
\text { FH-SS: } \quad \frac{\log p_{s}^{\mathrm{FH}}(\theta)}{\log p_{s}(\theta)}=M^{-1} .
$$

Since $\delta<1$, the benefit of FH-SS is larger; the difference is more drastic for small $\delta$, i.e., if the path loss exponent is large relative to the number of network dimensions. More details are available in [1].

\subsection{CSMA and Interference Cancellation}

\subsubsection{CSMA}

Channel access schemes that are based on carrier sensing aim at upper bounding the interference at a receiver by prohibiting nearby nodes to transmit. The effect of CSMA-type MAC schemes on the interference can be investigated by calculating the residual interference that stems from the transmitters outside the receiver's carrier sensing range. Assuming a carrier sensing range of $\rho$, we obtain the Laplace transform of the residual interference using a modified path loss law

$$
\tilde{\ell}(r)=r^{-\alpha} \mathbf{1}_{r>\rho},
$$

and following the same steps as in the calculation of the entire interference. In the non-fading case,

$$
\mathcal{L}_{I}(s)=\exp \left\{-\lambda c_{d}\left(s^{\delta} \gamma\left(1-\delta, s \rho^{-\alpha}\right)-\rho^{d}\left(1-\exp \left(-s \rho^{-\alpha}\right)\right)\right)\right\},
$$


where $\gamma(a, z)=\int_{0}^{z} \exp (-t) t^{a-1} \mathrm{~d} t$ is the lower incomplete gamma function. Since $\gamma(a, z)<\Gamma(a)$ for finite $z$, this is larger than the Laplace transform of the complete interference, as expected. For $\rho>0$, the mean and variance are finite and given by

$$
\begin{aligned}
\mathbb{E}(I) & =-\left.\frac{\mathrm{d}}{\mathrm{d} s} \log \left(\mathcal{L}_{I}(s)\right)\right|_{s=0}=\frac{\lambda c_{d} d}{\alpha-d} \rho^{d-\alpha} . \\
\operatorname{var}(I) & =\left.\frac{\mathrm{d}^{2}}{\mathrm{~d} s^{2}} \log \left(\mathcal{L}_{I}(s)\right)\right|_{s=0}=\frac{\lambda c_{d} d}{2 \alpha-d} \rho^{d-2 \alpha} .
\end{aligned}
$$

These results follow from the fact that

$$
\lim _{s \rightarrow 0} \frac{\gamma\left(1-\delta, s \rho^{-\alpha}\right)}{s^{1-\delta}}=\frac{\rho^{d-\alpha}}{1-\delta}
$$

In the fading case,

$$
\begin{aligned}
& \mathcal{L}_{I}(s)= \\
& \quad \exp \left\{-\lambda c_{d}\left(s^{\delta} \mathbb{E}_{h}\left(h^{\delta} \gamma\left(1-\delta, s h \rho^{-\alpha}\right)\right)-\rho^{d} \mathbb{E}_{h}\left(1-\exp \left(-s h \rho^{-\alpha}\right)\right)\right)\right\} .
\end{aligned}
$$

The expectation of the term with the incomplete gamma function can be evaluated numerically, or bounds can be used. In Rayleigh fading, the expectation of the exponential part is $\rho^{d} s /\left(s+\rho^{\alpha}\right)$, and for $\delta=1 / 2$,

$$
\mathbb{E}_{h}\left(h^{\delta} \gamma(1-\delta, s h c)\right)=\frac{\pi}{2}-\arctan \left(\frac{1}{\sqrt{s c}}\right)+\frac{\sqrt{s c}}{s c+1} .
$$

Hence for Rayleigh fading and $\delta=1 / 2$,

$$
\begin{aligned}
& \mathcal{L}_{I}(s)= \\
& \quad \exp \left\{-\lambda c_{d} \sqrt{s}\left(\frac{\pi}{2}-\arctan \left(\frac{1}{\sqrt{s \rho^{-\alpha}}}\right)+\frac{\sqrt{s \rho^{-\alpha}}}{s \rho^{-\alpha}+1}\right)+\frac{\lambda c_{d} \rho^{d} s}{s+\rho^{\alpha}}\right\},
\end{aligned}
$$

which confirms that the interference does not have a heavy tail for $\rho>0$.

Interpreting $\mathcal{L}_{I}(\theta)$ as the success probability in Rayleigh fading, the impact of CSMA on the outage can be quantified. Figure 3.4 shows the success probability for $\rho=1 / 2,1,2$ and ALOHA, for comparison. Strictly speaking, this formula is only an approximation, since the set 


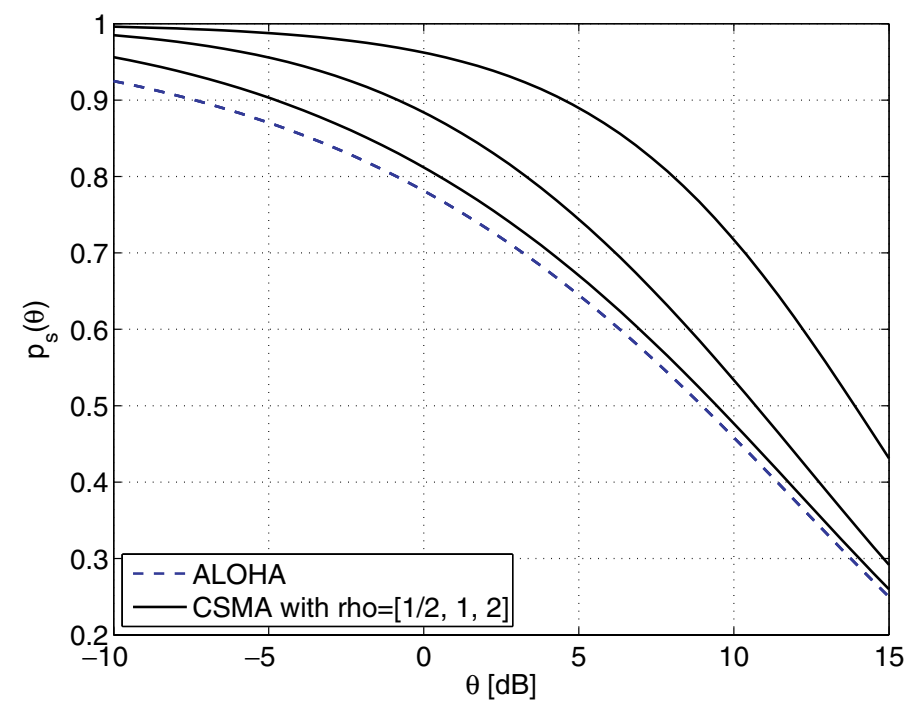

Fig. 3.4 Success probabilities $p_{s}(\theta)=\mathcal{L}_{I}(\theta)$ from (3.46) for ALOHA (dashed curve) and CSMA with guard zone radii $\rho=1 / 2,1,2$ for a two-dimensional network with $\alpha=4(\delta=$ $1 / 2$ ) and $\lambda=1 / 20$. The curve with the highest success probability is the one where $\rho=2$.

of transmitters no longer form a PPP under the CSMA model. Instead, it forms a hard-core point process [45], where a minimum distance is imposed between the transmitters. For relatively small guard zones, using the PPP as a model yields rather accurate results since the "distortion" in the point process outside the guard zone is minimal. The mean $\mathbb{E}(I)$ is the same as in the non-fading case, and the variance is twice as large. So Rayleigh fading doubles the variance in the interference.

CSMA-type MACs essentially create a guard zone around the receiver. This helps this particular receiver, but it also reduces the number of concurrent transmissions that are possible in the network, i.e., the spatial reuse. This trade-off is investigated in detail in [24]. Here we just note that a guard zone of radius $\rho$ reduces the density of transmitters by a thinning factor $\exp \left(-\lambda c_{d} \rho^{d}\right)$. One way to quantify the spatial density of successful transmissions is to multiply the density of transmitters with the success probability. For the parameters in Figure 3.4 , the thinning factors are $96 \%, 85 \%$, and $53 \%$ for $\rho=1 / 2,1,2$, 
respectively. For $\rho=1$, the product of success probability and density of transmitters is higher than in the ALOHA case as soon as $\theta>0$ dB. Generally, the optimum width of the guard zone depends on the reliability and energy efficiency requirements.

In [37], the authors analyzed the performance of CSMA in dense 802.11 networks. They used a Matern-type hard-core process to model the impact of CSMA on the node distribution.

\subsubsection{Interference Cancellation}

Multi-user receivers can achieve significantly higher performance in wireless networks. Successive interference cancellation (SIC) is a particularly appealing technique when the received powers from the users differ greatly $[47,38]$. In a large wireless network, one can expect substantial benefits if the interference from one or a few of the strongest interferers can be canceled. A similar effect as in CSMA can be achieved, albeit without the reduction in transmitter density. We have previously derived an expression for the distribution of the interference from each individual interferer (3.26); however, using this result in the present context is complicated by the fact that the distance distributions are not independent. The tail probabilities of the individual interference powers (3.27) tell us how heavy the tail remains if a certain number of nearby interferers are canceled, but again this does not help with analyzing the outage probabilities.

The Laplace transforms of the interference from the nearest $n$ transmitters is given in (3.19) for Rayleigh fading and $\delta=1 / 2$. Let $p_{s}(\theta, \mathcal{I})$, $\mathcal{I} \subset \mathbb{N}$, be the success probability if the $i$-th nearest interferers, $i \in \mathcal{I}$, are present and active. From $p_{s}(\theta,[n])=\mathcal{L}_{I_{n}}(\theta)$ we define as the $n$-th interferer's contribution to the outage

$$
p_{s}(\theta,\{n\}) \triangleq \frac{p_{s}(\theta,[n])}{p_{s}(\theta,[n-1])}
$$

and the success probability when the $k$ nearest interferers are canceled,

$$
p_{s}(\theta, \mathbb{N} \backslash[k]) \triangleq \frac{p_{s}(\theta, \mathbb{N})}{p_{s}(\theta,[k])} .
$$

Using (3.19), these probabilities can easily be found numerically. Figure 3.5 (left) shows the success probabilities for $k=0$ (no SIC) and 

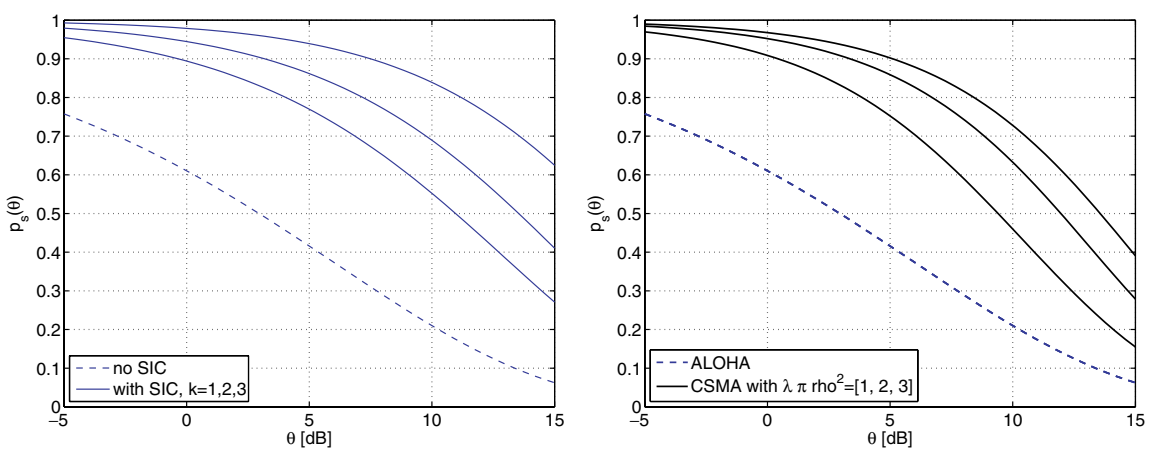

Fig. 3.5 Left: Success probabilities $p_{s}(\theta)$ from (3.19) without SIC (dashed curve) and with SIC of the nearest 1,2 , and 3 interferers canceled for a two-dimensional network with $\alpha=4$ $(\delta=1 / 2)$ and $\lambda=1 / 10$. The curve with the highest success probability is the one where the nearest 3 interferers are canceled. Right: For comparison, the success probabilities for CSMA with sensing radii $\rho$ chosen such that the mean number of interferers inside that radius is $1,2,3$. So, on average, CSMA removes 1,2 , or 3 interferers.

$k=1,2,3$. It can be seen that canceling just the first interferer has a drastic impact. If instead of the $k$ nearest interferers the $k$ strongest ones (including fading) were canceled, the SIC gain would be slightly larger even.

A different route was taken in [48], where the transmission capacity framework was employed to investigate the effect of SIC on the outage probabilities. Instead of determining the benefits of canceling the $k$ nearest interferers, they defined a cancellation radius $\rho_{k} \triangleq \sqrt{k /(\lambda \pi)}$ (two-dimensional network) and assumed that all interferers within this radius are canceled. The model then more closely resembles the CSMA model with a guard zone $\rho$. They showed that the benefits are significant already for small $k$ if spread-spectrum techniques are used, but that cancellation needs to be almost perfect; if it is imperfect, i.e., if the residual interference $\epsilon I_{k}$ from the $k$ suppressed interferers, the gains are substantially smaller even if $\epsilon \ll 1$.

In Figure 3.5 (right), for comparison, the success probabilities for CSMA with guard zone radii $\rho_{k}$ for $k=1,2,3$ such that on average 1,2 , or 3 interferers are blocked, are shown. These curves show good agreement with the ones for SIC. The difference stems from the fact that with SIC, there may still be interferers relatively close, while with CSMA, it is not guaranteed that any interferer is muted at all. 


\subsection{Interference Correlation}

From the previous section we observe that the interference distribution in a Poisson network does not depend on the spatial location due to the stationarity of the PPP. Even though the interference distribution is identical on the entire plane (in the two-dimensional case), the interference is not independent across the plane. This is because the interference is caused by common randomness, namely the point process $\Phi$. The spatial and temporal correlation of the interference is often ignored in the literature, or removed by the assumption that a new realization of the point process is drawn in each time slot [46]. This is clearly not the case in practical networks, and thus there is a need to analyze the correlation structure of the interference if the point process realization does not change. This is the topic of this section.

Since each node in a wireless network uses a MAC protocol to decide whether to transmit or receive, the transmitting set in time slot $k$, denoted as $\Phi_{k}$, changes with time $k$ but is always a subset of $\Phi$; as a consequence, the interference becomes correlated over time because of the common randomness $\Phi$. In this section we consider ALOHA as the MAC protocol in which each node transmits with probability $p$ and receive with $1-p$ independently of other nodes. We observe that ALOHA as a MAC protocol introduces no correlations since the transmitters are independently chosen in space and time. Nevertheless the presence of the common randomness $\Phi$ causes the interference to be temporally correlated.

When $\ell(x)=\|x\|^{-\alpha}$ the interference is heavy-tailed with parameter $2 / \alpha$. As mentioned earlier the average interference and the higher moments are not finite since an interfering transmitter can be very close to the origin (where the interference is calculated). The correlation coefficient cannot be defined when the moments are not finite. Hence we use a bounded path loss model and calculate the spatio-temporal correlation coefficient for $\ell(x)=\|x\|^{-\alpha}$ as a limiting case.

$\ell(x)$ is assumed to have the following properties:

(1) Depends only on $\|x\|$.

(2) Monotonically decreases with $\|x\|$. 
(3) Integrable:

$$
\int_{0}^{\infty} x \ell(x) \mathrm{d} x<\infty
$$

For example, a valid path loss model is given by

$$
\ell_{\epsilon}(x)=\frac{1}{\epsilon+\|x\|^{\alpha}}, \epsilon \in(0, \infty), \alpha>2 .
$$

The standard singular path loss model $\ell(x)=\|x\|^{-\alpha}$ is obtained by taking the limit $\lim _{\epsilon \rightarrow 0} \ell_{\epsilon}(x)$. Since in ALOHA, each node makes an independent decision to transmit at every time instant, the set of transmitters at a time instant is a thinned version of the original PPP $\Phi$, and the resulting transmitter process at time instant $k, \Phi_{k}$, is again a PPP. This can be easily verified by considering the void probability: For any bounded set $B \subset \mathbb{R}^{2}$, we have

$$
\begin{aligned}
\mathbb{P}\left(\Phi_{k}(B)=0\right) & =\mathbb{E} \prod_{x \in \Phi \cap B} \mathbf{1}(\mathrm{x} \text { not a transmitter at time } k) \\
& \stackrel{(a)}{=} \prod_{x \in \Phi \cap B} \mathbb{P}(\mathrm{x} \text { not a transmitter at time } k) \\
& =\mathbb{E}\left[(1-p)^{\Phi(B)}\right] \\
& \stackrel{(b)}{=} \exp (-\lambda p|B|) .
\end{aligned}
$$

(a) follows since each node decides to not transmit independently from each other. Since $\Phi$ is a PPP, $\Phi(B)$ is a Poisson random variable with mean $\lambda|B|,(b)$ follows from the moment generating function of the Poisson random variable. From the above we see that the void probability of the transmitting process at time $k$ is $\exp (-p \lambda|B|)$, which corresponds to that of a PPP. We also observe that $\Phi_{k}$ has density $p \lambda$ which is intuitive. The interference at time instant $k$ and (spatial) location $z$ is given by

$$
I_{k}(z)=\sum_{\mathrm{x} \in \Phi} \mathbf{1}\left(\mathrm{x} \in \Phi_{k}\right) h_{\mathrm{x} z}(k) \ell(\mathrm{x}-z) .
$$

We assume that the fading is independent across time (block fading) and space. 


\subsubsection{Spatio-Temporal Correlation of the Interference}

We calculate the correlation coefficient of the random variables, $I_{k}(u)$ and $I_{l}(v), k \neq l, u \neq v$. In order to calculate the joint moments of the random variables, the joint Laplace transform of the random variables $I_{k}(u)$ and $I_{l}(v)$ will be used.

Theorem 3.1. The joint Laplace transform of $I_{k}(u)$ and $I_{l}(v), k \neq l$, is

$$
\mathcal{L}\left(s_{1}, s_{2}\right)=\exp \left(-\lambda \int_{\mathbb{R}^{2}}\left[1-\xi\left(s_{1}, u-x\right) \xi\left(s_{2}, v-x\right)\right] \mathrm{d} x\right),
$$

where

$$
\xi(s, x)=1-p+p \mathcal{L}_{h}(s \ell(x)),
$$

and $\mathcal{L}_{h}$ denotes the Laplace transform of the fading process.

Proof. The interference at time $k$ and location $u \in \mathbb{R}^{2}$ is given by

$$
I_{k}(u)=\sum_{\mathrm{x} \in \Phi_{k}} h_{\mathrm{x} u}(k) \ell(\mathrm{x}-u),
$$

and the interference at $v \in \mathbb{R}^{2}$ at time $l$ is

$$
I_{l}(v)=\sum_{\mathrm{y} \in \Phi_{l}} h_{\mathrm{y} v}(l) \ell(\mathrm{y}-v) .
$$

So the joint Laplace transform is

$$
\mathcal{L}\left(s_{1}, s_{2}\right)=\mathbb{E} \exp \left[-s_{1} \sum_{\mathbf{x} \in \Phi_{k}} h_{\mathrm{x} u}(k) \ell(\mathrm{x}-u)-s_{2} \sum_{\mathbf{y} \in \Phi_{l}} h_{\mathrm{y} v}(l) \ell(\mathrm{y}-v)\right] .
$$

Rewriting this as a product,

$$
\begin{aligned}
\mathcal{L}\left(s_{1}, s_{2}\right)= & \mathbb{E} \prod_{\mathrm{x} \in \Phi} \exp \left(-s_{1} \mathbf{1}\left(\mathrm{x} \in \Phi_{k}\right) h_{\mathrm{x} u}(k) \ell(\mathrm{x}-u)\right) \\
& \times \exp \left(-s_{2} \mathbf{1}\left(\mathrm{x} \in \Phi_{l}\right) h_{\mathrm{x} v}(l) \ell(\mathrm{x}-v)\right) .
\end{aligned}
$$

Since in ALOHA each node decides to transmit independently of other nodes and across time, taking the expectation with respect to ALOHA,

$$
\begin{aligned}
\mathcal{L}\left(s_{1}, s_{2}\right)= & \mathbb{E} \prod_{\times \in \Phi}\left[1-p+p \exp \left(-s_{1} h_{\times u}(k) \ell(\mathrm{x}-u)\right)\right] \\
& \times\left[1-p+p \exp \left(-s_{2} h_{\times v}(l) \ell(\mathrm{x}-v)\right)\right] .
\end{aligned}
$$


Since fading is assumed to be independent across time and space, moving the expectation with respect to fading inside we get

$$
\begin{aligned}
\mathcal{L}\left(s_{1}, s_{2}\right) & =\mathbb{E} \prod_{\mathbf{x} \in \Phi}\left[1-p+p \mathcal{L}_{h}\left(s_{1} \ell(\mathrm{x}-u)\right)\right]\left[1-p+p \mathcal{L}_{h}\left(s_{2} \ell(\mathrm{x}-v)\right)\right] \\
& =\mathbb{E} \prod_{\mathrm{x} \in \Phi} \xi\left(s_{1}, \mathrm{x}-u\right) \xi\left(s_{2}, \mathrm{x}-v\right) \\
& \stackrel{(c)}{=} \exp \left(-\lambda \int_{\mathbb{R}^{2}}\left[1-\xi\left(s_{1}, x-u\right) \xi\left(s_{2}, x-v\right)\right] \mathrm{d} x\right) .
\end{aligned}
$$

The last step follows from the PGFL of the PPP (A.3).

The above proof can be generalized to obtain the joint Laplace transform of the $m$ random variables $\left\{I_{k_{1}}\left(u_{1}\right), \ldots, I_{k_{m}}\left(u_{m}\right)\right\}, k_{1} \neq$ $k_{2}, \ldots, \neq k_{m}$ and $u_{1} \neq u_{2}, \ldots, \neq u_{m}$ :

$$
\mathcal{L}_{I_{\mathcal{K}}}\left(s_{1}, \ldots, s_{m}\right)=\exp \left(-\lambda \int_{\mathbb{R}^{2}}\left[1-\prod_{i=1}^{m} \xi\left(s_{i}, x-u_{i}\right)\right] \mathrm{d} x\right) .
$$

In the above theorem, we have considered the interference at two different time slots and at different spatial locations.

By a similar approach as in the theorem above, the joint Laplace transform of the random variables $I_{k}(u)$ and $I_{k}(v)$, i.e., interference at the same time instant but at different locations can be shown to be

$\mathcal{L}_{I(u) I(k)}\left(s_{1}, s_{2}\right)=\exp \left(-\lambda \int_{\mathbb{R}^{2}}\left[1-\mathcal{L}_{h}\left(s_{1} \ell(x-u)\right) \mathcal{L}_{h}\left(s_{2} \ell(x-v)\right)\right] \mathrm{d} x\right)$.

The mean of the interference is

$$
\begin{aligned}
\mathbb{E} I_{k}(u) & =-\left.\frac{\partial}{\partial s_{1}} \mathcal{L}\left(s_{1}, 0\right)\right|_{s_{1}=0} \\
& =-\left.\frac{\partial}{\partial s_{1}} \exp \left(-\lambda \int_{\mathbb{R}^{2}}\left[1-\xi\left(s_{1}, x-u\right) \xi(0, x-v)\right] \mathrm{d} x\right)\right|_{s_{1}=0} \\
& =\left.\lambda p \int_{\mathbb{R}^{2}} \frac{\partial}{\partial s_{1}} \mathcal{L}_{h}\left(s_{1} \ell(x-u)\right) \mathrm{d} x\right|_{s_{1}=0} \\
& =p \lambda \mathbb{E}[h] \int_{\mathbb{R}^{2}} \ell(x-u) \mathrm{d} x \\
& =p \lambda \int_{\mathbb{R}^{2}} \ell(x) \mathrm{d} x
\end{aligned}
$$


since $\mathbb{E}[h]=1$. The second moment of the interference is

$$
\mathbb{E}\left[I_{k}(u)^{2}\right]=\left.\frac{\partial^{2}}{\partial^{2} s_{1}} \mathcal{L}\left(s_{1}, 0\right)\right|_{s_{1}=0}
$$

and by simple algebraic manipulations, the second moment

$$
\begin{aligned}
\mathbb{E}\left[I_{k}(u)^{2}\right]= & p \mathbb{E}\left[h^{2}\right] \lambda \int_{\mathbb{R}^{2}} \ell^{2}(x) \mathrm{d} x \\
& +p^{2} \mathbb{E}[h]^{2} \lambda^{2} \int_{\mathbb{R}^{2}} \int_{\mathbb{R}^{2}} \ell(x) \ell(y) \mathrm{d} x \mathrm{~d} y .
\end{aligned}
$$

is obtained. When the path loss is given by $\ell_{\epsilon}(x)$ and the fading is Rayleigh, we can use the above theorem to obtain the Laplace transform of the interference as

$$
\mathcal{L}_{I_{\Phi}}(s)=\exp \left(-\pi \lambda p s(\epsilon+s)^{\delta-1} \frac{\pi \delta}{\sin (\pi \delta)}\right),
$$

where $\delta=2 / \alpha$.

The mean and the variance do not depend on either the position or the time index. When the fading follows a Nakagami- $m$ distribution and the path loss model is given by $\ell_{\epsilon}(x)$, the variance of the interference follows from (3.51) and (3.52) and is given by

$$
\operatorname{var}\left[I_{k}(u)\right]=\frac{p \lambda \pi}{\epsilon^{2-\delta}} \frac{\pi \delta}{\sin (\pi \delta)}(1-\delta)\left(1+m^{-1}\right)
$$

We observe that the variance is maximum, when the fading is Rayleigh and the minimum exactly half of the maximum when there is no fading (i.e., $m=\infty$ ). As expected, the mean and the variance diverge when $\epsilon=0$. The mean of the product of $I_{k}(u)$ and $I_{l}(v)$ at times $k$ and $l, k \neq l$, which is required for the calculation of the correlation coefficient, is

$$
\begin{aligned}
\mathbb{E}\left[I_{k}(u) I_{l}(v)\right] & =\left.\frac{\partial^{2}}{\partial s_{2} \partial s_{1}} \mathcal{L}\left(s_{1}, s_{2}\right)\right|_{\left(s_{1}, s_{2}\right)=(0,0)} \\
& =p^{2} \lambda \int_{\mathbb{R}^{2}} \ell(x-u) \ell(x-v) \mathrm{d} x+\lambda^{2} p^{2}\left(\int_{\mathbb{R}^{2}} \ell(x) \mathrm{d} x\right)^{2}
\end{aligned}
$$

Lemma 3.2. The spatio-temporal correlation coefficient of the interferences $I_{k}(u)$ and $I_{l}(v), k \neq l$, for ALOHA and path loss functions $\ell(x)$ 
satisfying (3.47) is

$$
\zeta(u, v)=\frac{p \int_{\mathbb{R}^{2}} \ell(x) \ell(x-\|u-v\|) \mathrm{d} x}{\mathbb{E}\left[h^{2}\right] \int_{\mathbb{R}^{2}} \ell^{2}(x) \mathrm{d} x} .
$$

Proof. Since $I_{k}(u)$ and $I_{l}(v)$ are identically distributed, we have

$$
\zeta(u, v)=\frac{\mathbb{E}\left[I_{k}(u) I_{l}(v)\right]-\mathbb{E}\left[I_{k}(u)\right]^{2}}{\mathbb{E}\left[I_{k}(u)^{2}\right]-\mathbb{E}\left[I_{k}(u)\right]^{2}}
$$

By substituting for the above quantities we have

$$
\begin{aligned}
\zeta(u, v) & =\frac{p \int_{\mathbb{R}^{2}} \ell(x-u) \ell(x-v) \mathrm{d} x}{\mathbb{E}\left[h^{2}\right] \int_{\mathbb{R}^{2}} \ell^{2}(x) \mathrm{d} x} \\
& \stackrel{(a)}{=} \frac{p \int_{\mathbb{R}^{2}} \ell(x) \ell(x-\|u-v\|) \mathrm{d} x}{\mathbb{E}\left[h^{2}\right] \int_{\mathbb{R}^{2}} \ell^{2}(x) \mathrm{d} x},
\end{aligned}
$$

where (a) follows by substituting $y=x-u$ and the fact that $\ell(x)$ depends only on $\|x\|$.

We observe that the correlation coefficient does not depend on $k$ and $l$ and is equal for any two time instants. From (3.50) we obtain the following lemma on the spatial correlation:

Lemma 3.3. The spatial correlation coefficient of the interference, i.e., the correlation coefficient of $I_{k}(u)$ and $I_{k}(v), u \neq v$ is

$$
\zeta_{s}(u, v)=\frac{\int_{\mathbb{R}^{2}} \ell(x) \ell(x-\|u-v\|) \mathrm{d} x}{\mathbb{E}\left[h^{2}\right] \int_{\mathbb{R}^{2}} \ell^{2}(x) \mathrm{d} x} .
$$

By setting $\|u-v\|=0$ in Lemma 3.2, we obtain the temporal correlation coefficient:

Lemma 3.4. The temporal correlation coefficient for ALOHA is

$$
\zeta_{t}=\frac{p}{\mathbb{E}\left[h^{2}\right]},
$$

independent of $\ell(x)$. When the fading is Nakagami- $m$, the correlation coefficient is $\zeta_{t}=\frac{p m}{m+1}$. In particular, for $m=1$ (Rayleigh fading), the 
temporal correlation coefficient is $p / 2$ and for $m \rightarrow \infty$ (no fading), it is $p$.

Hence the correlation increases with increasing $m$, i.e., fading decreases correlation which is intuitive. Note that in the above derivation, $\int_{\mathbb{R}^{2}} \ell^{2}(x) \mathrm{d} x$ is not defined when $\ell(x)=\|x\|^{-\alpha}$, but we can use $\ell_{\epsilon}(x)$ and take $\epsilon \rightarrow 0$. We now find the correlation for the singular path loss model as a limit of $\ell_{\epsilon}(x)$.

Lemma 3.5. Let the path loss model be given by $\ell_{\epsilon}(x)=1 /(\epsilon+$ $\left.\|x\|^{\alpha}\right)$. When $u \neq v$, correlation is

$$
\lim _{\epsilon \rightarrow 0} \zeta_{s}(u, v)=0
$$

Proof. We have

$$
\begin{aligned}
& \zeta_{s}=\lim _{\epsilon \rightarrow 0} \frac{\int_{\mathbb{R}^{2}} \ell_{\epsilon}(x-u) \ell_{\epsilon}(x-v) \mathrm{d} x}{\mathbb{E}\left[h^{2}\right] \int_{\mathbb{R}^{2}} \ell_{\epsilon}^{2}(x) \mathrm{d} x} \\
& \stackrel{(a)}{=} \lim _{\epsilon \rightarrow 0} \frac{p \int_{\mathbb{R}^{2}} \frac{1}{1+\left\|x-u \epsilon^{-1 / \alpha}\right\|^{\alpha}} \frac{1}{1+\left\|x-v \epsilon^{-1 / \alpha}\right\|^{\alpha}} \mathrm{d} x}{\mathbb{E}\left[h^{2}\right] \int_{\mathbb{R}^{2}}\left(\frac{1}{1+\|x\|^{\alpha}}\right)^{2} \mathrm{~d} x} \\
&=0,
\end{aligned}
$$

where $(a)$ follows from a change of variables.

The correlation coefficient being 0 is an artifact of the singular path loss model. When the path loss is $\|x\|^{-\alpha}$, the correlation coefficient is determined by the first-order statistics, which are dominated by the interference in an $a$-neighborhood of $u, v, a>0$. See Figure 3.6. For $a<\|u-v\| / 2$, the interferences $I_{u}^{a}$ and $I_{v}^{a}$ are independent.

Even when $\zeta(u, v)=0, I_{k}(u)$ and $I_{l}(v)$ are not independent. Indeed the joint Laplace transform obtained in Theorem 3.1 does not factorize for the singular path loss model. In Figure 3.7, the spatial correlation is plotted as a function of $\|u-v\|$ for different $\epsilon$. 


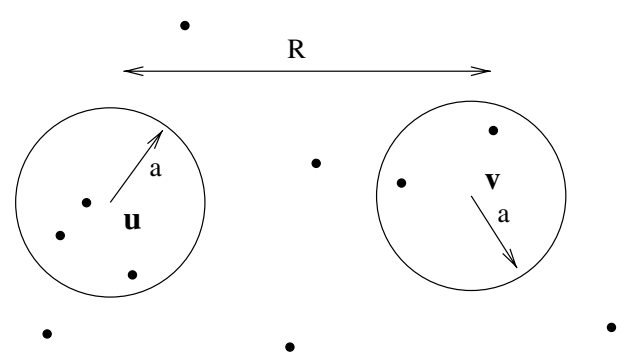

Fig. 3.6 For any two points $u$ and $v$, choose $a<\|u-v\| / 2$. Then the locations of the nodes in $b(u, a)$ and $b(v, a)$ are independent. Since the interference is dominated by the nearest interferers when the path loss is singular, $I_{u}^{a}$ and $I_{v}^{a}$ are independent, causing the correlation to be zero.

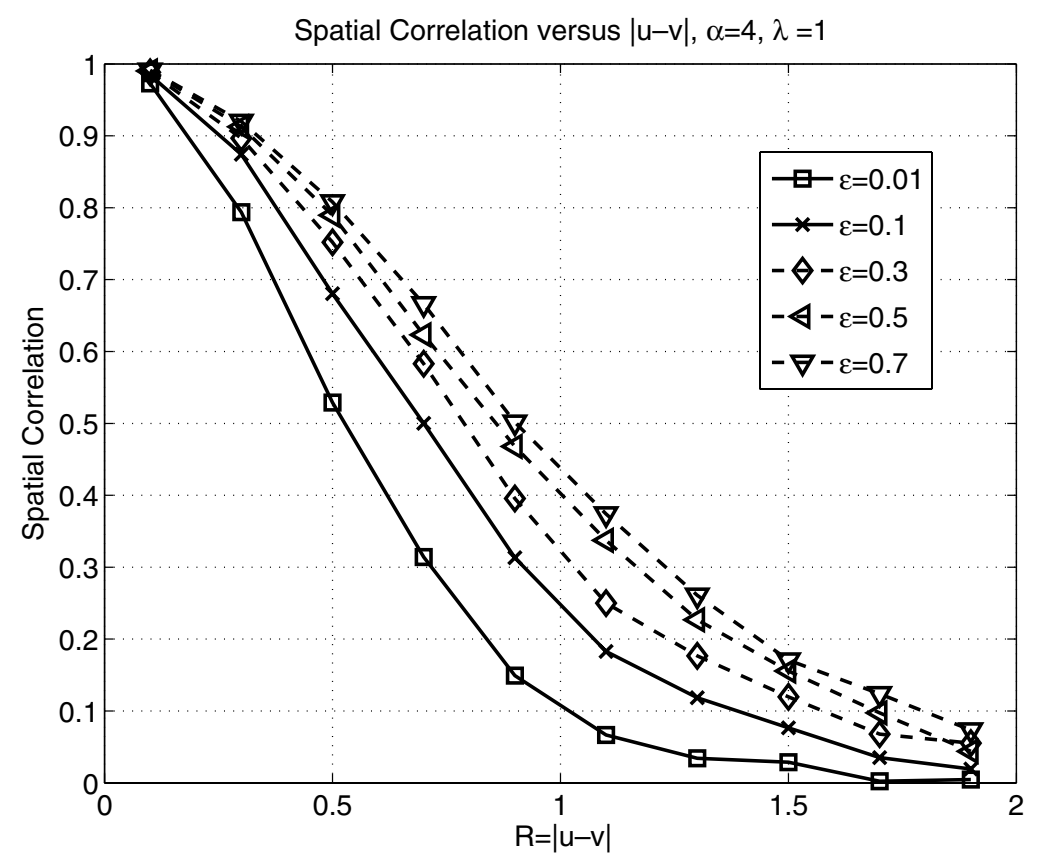

Fig. 3.7 Normalized spatial correlation $\zeta(u, v) / p$ versus $\|u-v\|$, when the path loss model is given by $\ell_{\epsilon}(x), \lambda=1$ and $\alpha=4$. We observe that $\zeta_{s}(u, v) \rightarrow 0, u \neq v$, for $\epsilon \rightarrow 0$ (from [14] (C) 2009 IEEE).

\subsubsection{Temporal Correlation of Link Outages}

In the standard analyses of retransmissions in a wireless ad hoc system, the link failures are assumed to be uncorrelated across time. But this 
is not so, since the interference is temporally correlated. We now provide the conditional probability of link formation assuming a successful transmission.

We assume that a transmitter at the origin has a destination located at $z \in \mathbb{R}^{2}$. Let $A_{k}$ denote the event that the origin is able to connect to its destination $z$ at time instant $k$, i.e.,

$$
\mathrm{SIR}=\frac{h_{o z}(k) \ell(z)}{I_{k}(z)}>\theta .
$$

For simplicity we shall assume the fading is Rayleigh (similar methods can be used for Nakagami- $m$ ). We now provide the joint probability of success $\mathbb{P}\left(A_{k}, A_{l}\right), k \neq l$. We have

$$
\begin{aligned}
\mathbb{P}\left(A_{k}, A_{l}\right) & \\
& =\mathbb{P}\left(h_{o z}(k)>\theta_{z} I_{k}(z), h_{o z}(l)>\theta_{z} I_{l}(z)\right) \\
& \stackrel{(a)}{=} \mathbb{E}\left[\exp \left(-\theta_{z} I_{k}(z)\right) \exp \left(-\theta_{z} I_{l}(z)\right)\right] \\
& =\mathbb{E}\left[\exp \left(-\theta_{z} \sum_{\mathrm{x} \in \Phi} \ell(\mathrm{x})\left[\mathbf{1}\left(\mathrm{x} \in \Phi_{k}\right) h_{\mathrm{x} z}(k)+\mathbf{1}\left(\mathrm{x} \in \Phi_{l}\right) h_{\mathrm{x} z}(l)\right]\right)\right] \\
& \stackrel{(b)}{=} \mathbb{E}\left[\prod_{x \in \Phi}\left(\frac{p}{1+\theta_{z} \ell(\mathrm{x})}+1-p\right)^{2}\right] \\
& \stackrel{(c)}{=} \exp \left(-\lambda \int_{\mathbb{R}^{2}} 1-\left(\frac{p}{1+\theta_{z} \ell(x)}+1-p\right)^{2} \mathrm{~d} x\right),
\end{aligned}
$$

where $\theta_{z}=\theta / \ell(z)$. (a) follows from the independence of $h_{o z}(k)$ and $h_{o z}(l), \quad k \neq l,(b)$ follows by taking the average with respect to $h_{x z}(k), h_{x z}(l)$ and ALOHA, (c) follows from the probability generating functional of the PPP. Similarly, we have

$$
\mathbb{P}\left(A_{l}\right)=\exp \left(-\lambda \int_{\mathbb{R}^{2}} 1-\left(\frac{p}{1+\theta_{z} \ell(x)}+1-p\right) \mathrm{d} x\right) .
$$

Hence the conditional probability is

$$
\mathbb{P}\left(A_{k} \mid A_{l}\right)=\exp \left(-\lambda \int_{\mathbb{R}^{2}} \frac{p \theta_{z} \ell(x)}{1+\theta_{z} \ell(x)}-\left(\frac{p \theta_{z} \ell(x)}{1+\theta_{z} \ell(x)}\right)^{2} \mathrm{~d} x\right) .
$$


The ratio of conditional and the unconditional probability is given by

$$
\begin{aligned}
\frac{\mathbb{P}\left(A_{k} \mid A_{l}\right)}{\mathbb{P}\left(A_{l}\right)} & =\frac{\mathbb{P}\left(A_{k}, A_{l}\right)}{\mathbb{P}\left(A_{l}\right)^{2}} \\
& =\exp \left(\lambda p^{2} \int_{\mathbb{R}^{2}}\left(\frac{\theta_{z} \ell(x)}{1+\theta_{z} \ell(x)}\right)^{2} \mathrm{~d} x\right) \\
& >1
\end{aligned}
$$

When $\ell(x)=\|x\|^{-\alpha}$, we have

$$
\frac{\mathbb{P}\left(A_{k} \mid A_{l}\right)}{\mathbb{P}\left(A_{l}\right)}=\exp \left(p^{2} \lambda \pi\|z\|^{2}(1-\delta) \frac{\pi \delta}{\sin (\pi \delta)} \theta^{\delta}\right)
$$

where $\delta=2 / \alpha$. In Figures 3.8 and 3.9, we plot the conditional and the unconditional link success probabilities versus the ALOHA parameter, $p$ and $z$, respectively.

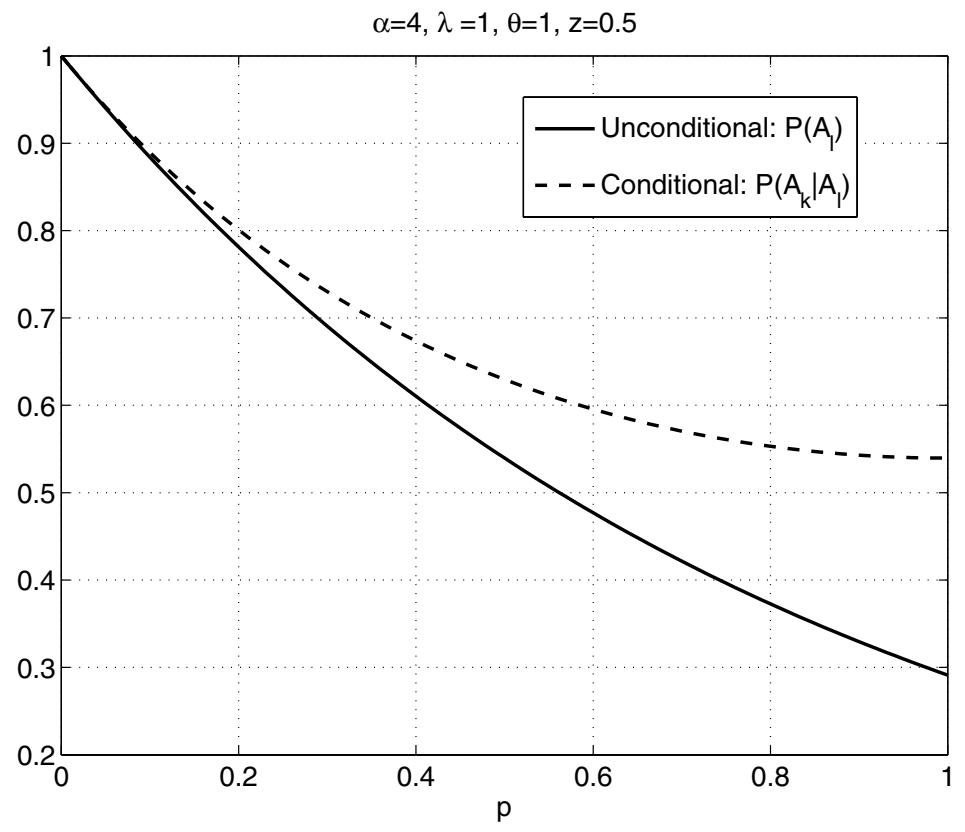

Fig. 3.8 $\mathbb{P}\left(A_{k} \mid A_{l}\right)$ and $\mathbb{P}\left(A_{l}\right)$ versus the ALOHA parameter $p . \lambda=1, \ell(x)=\|x\|^{-4}, z=0.5$, $\theta=1$ (from [14] (C) 2009 IEEE). 


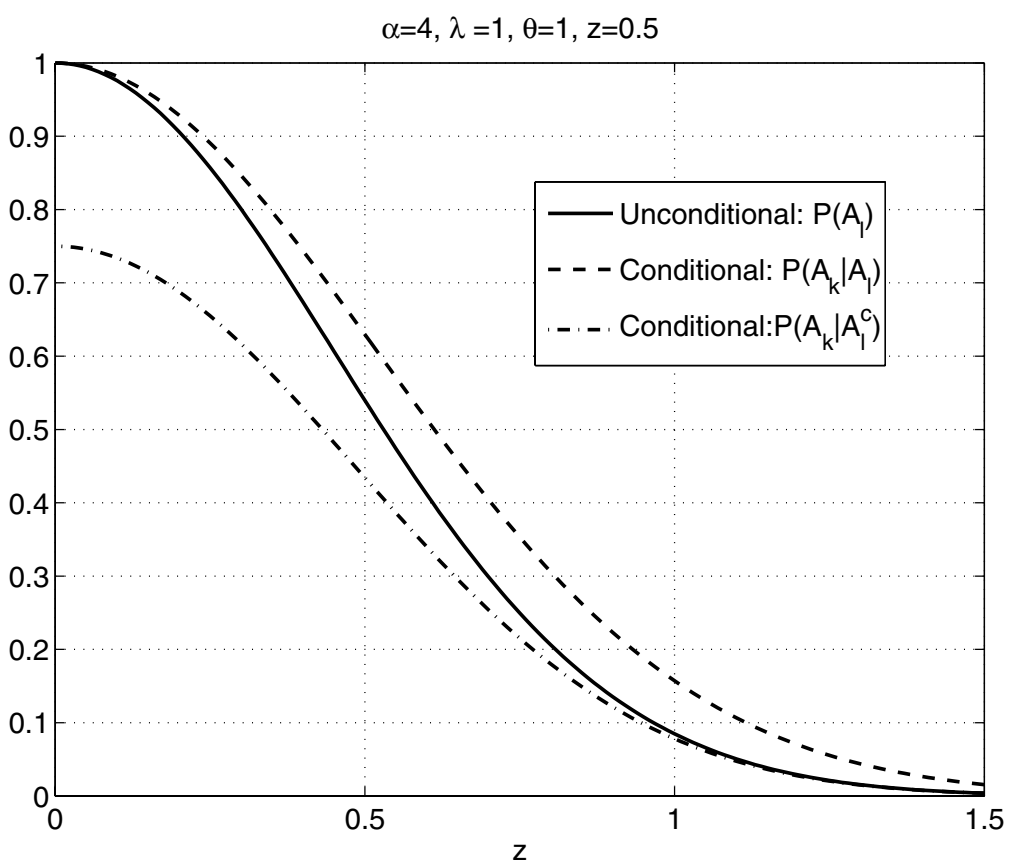

Fig. $3.9 \mathbb{P}\left(A_{k} \mid A_{l}\right)$ and $\mathbb{P}\left(A_{l}\right)$ versus the distance $z$. $\lambda=1, \ell(x)=\|x\|^{-4}, p=0.5, \theta=1$.

We make the following observations:

(1) The link formation is correlated across time (see (3.58)).

(2) If a transmission succeeds at a time instant $k$, there is a higher probability that a transmission succeeds at a time instant $l$.

(3) From (3.58), we also have $\mathbb{P}\left(A_{k}^{c} \mid A_{l}^{c}\right)>\mathbb{P}\left(A_{l}^{c}\right)$. So a link in outage is always more likely to be in outage; hence the retransmission strategy should reduce the rate of transmission or change the density of transmitters rather than retransmit "blindly".

(4) $\frac{\mathbb{P}\left(A_{k} \mid A_{l}\right)}{\mathbb{P}\left(A_{l}\right)}$ always increases with $\theta, \lambda, p$, and $\|z\|$. The increase in $\lambda$ and $p$ is because of the larger transmit set due to which the probability of the same subset of nodes transmitting at different times increases, thereby causing more correlation. When $\theta$ is large, the outage is a result of the interfering 


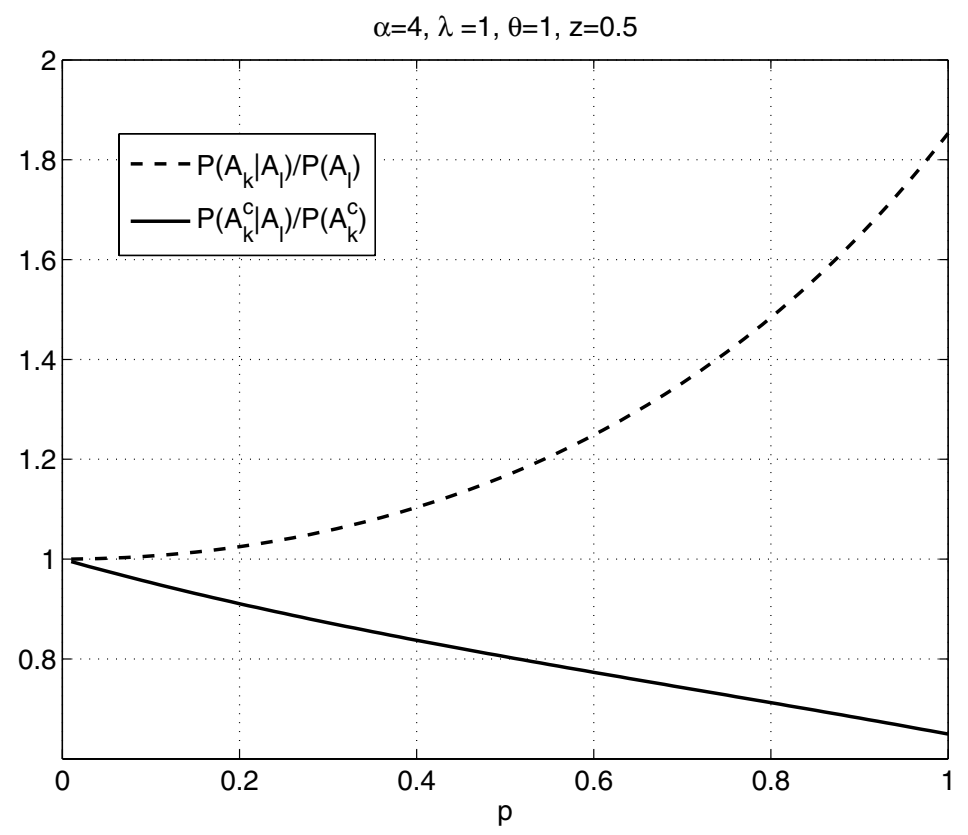

Fig. 3.10 $\frac{\mathbb{P}\left(A_{k} \mid A_{l}\right)}{\mathbb{P}\left(A_{l}\right)}$ and $\frac{\mathbb{P}\left(A_{k}^{c} \mid A_{l}\right)}{\mathbb{P}\left(A_{k}^{c}\right)}$ versus the ALOHA parameter $p . \lambda=1, \ell(x)=\|x\|^{-4}$, $z=0.5, \theta=1$.

transmissions caused by a larger number of nodes. Hence by a similar reasoning as above, the correlation increases.

(5) From Figure 3.10 it follows that $\frac{\mathbb{P}\left(A_{k} \mid A_{l}\right)}{\mathbb{P}\left(A_{l}\right)}$ increases with $p$ as evaluated. In the same figure, $\frac{\mathbb{P}\left(A_{k}^{c} \mid A_{l}\right)}{\mathbb{P}\left(A_{k}^{c}\right)}$ is plotted as a function of $p$, and we observe that it decreases with $p$. 


\section{4}

\section{Interference in Poisson Cluster Networks}

In the previous section we have analyzed the interference when the underlying transmitter set is a PPP. While this may be the case for certain networks, it is much more likely that the node distribution is not "completely spatially random" (CSR), i.e., that nodes are either clustered or more regularly distributed. Moreover, even if the complete set of nodes constitutes a PPP, the subset of active nodes may not be homogeneously Poisson. Instead, the transmitting node set may be clustered or regularly arranged (see Section 2).

The clustering of transmitting nodes may be due to geographical factors; for example, in a social gathering, people tend to cluster into small groups, or in a battlefield, the soldiers move in small groups in a coordinated fashion, or in a down-town region, wireless nodes are clustered in buildings, or in a cognitive network, the active cognitive users tend to be clustered. The clustering may also be "artificially" induced by MAC protocols. We denote the former as geographical clustering and the latter as logical clustering. See Figure 4.1. Also the benefits of randomly arranged transmissions as compared to clustered transmissions are not a priori clear. In this section we analyze the interference and outage properties when the transmitters are clustered as a Poisson 


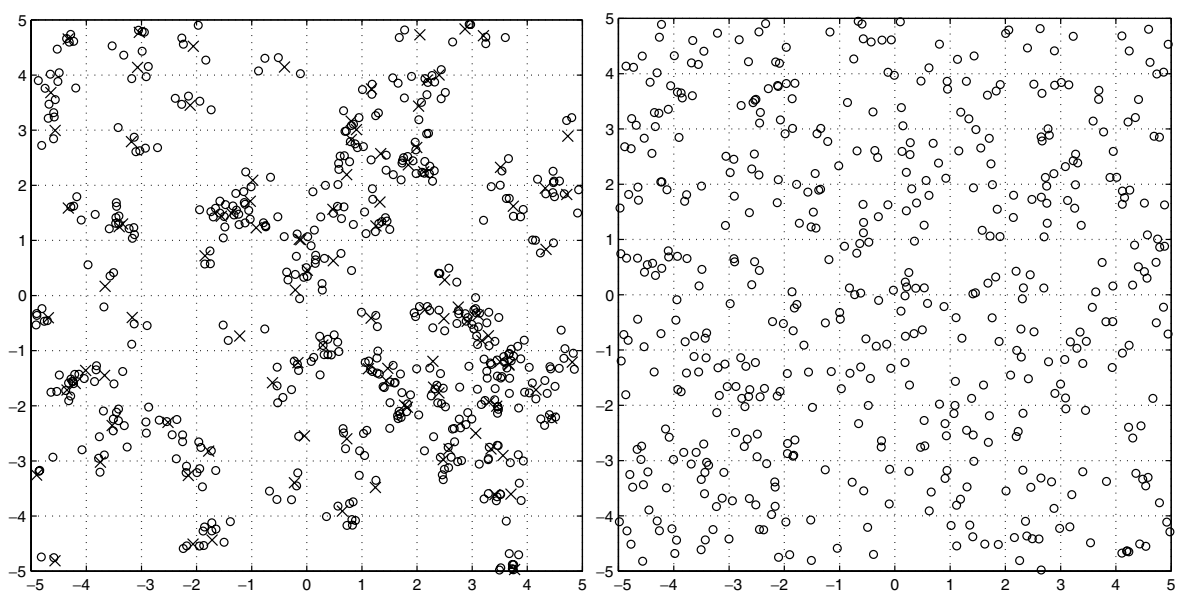

Fig. 4.1 (Left) Thomas cluster process with parameters $\lambda_{p}=1, \bar{c}=5$, and $\sigma=0.2$. The crosses indicate the parent points. (Right) PPP with the same intensity $\lambda=5$ for comparison (from [13] (c) 2009 IEEE).

cluster process and analyze the benefits of clustering over random transmissions.

System Model. The location of transmitting nodes is modeled as a stationary and isotropic Poisson cluster process [45] $\Phi$ on $\mathbb{R}^{2}$. We assume that the intended transmitter (a part of the transmit point process $\Phi$ ) is located at the origin, and its receiver (where we measure the interference) is located at $z$ with $\|z\|=R$. The receiver is not considered a part of the process. ${ }^{1}$ See Figure 4.2 .

Each transmitter is assumed to transmit at unit power and the power received by a receiver located at $z$ due to a transmitter at $x$ is modeled as $h_{x} \ell(x-z)$, where $h_{x}$ is the power fading coefficient associated with the channel between the nodes $x$ and $z$, assumed to be independent and exponentially distributed (Rayleigh fading). We assume that the path loss model $\ell(x): \mathbb{R}^{2} \backslash\{o\} \rightarrow \mathbb{R}^{+}$is a continuous, positive, non-increasing function of $\|x\|$. The interference at point $z$ is

${ }^{1}$ If the receivers were a part of the process, we would lose the notion of the common distance that information travelled and it is not a priori clear how to choose the transmit-receive pairs. 


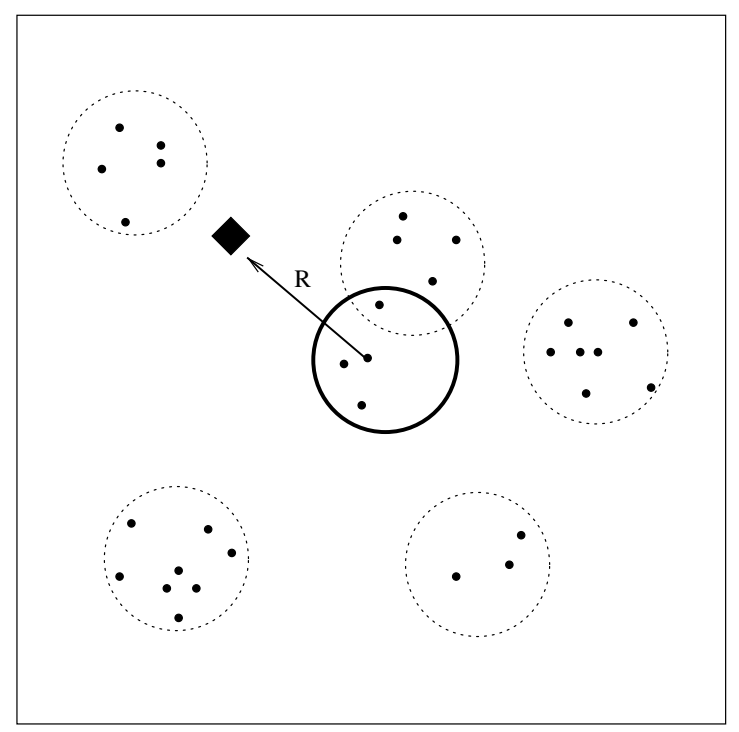

Fig. 4.2 Illustration of the system model for a Matern cluster process. The small black dots represent the transmitters, which form a Matern cluster process $\Phi$. We condition on the event that there is a node of $\Phi$ at the origin which we denote as the intended transmitter. The receiver (denoted by a square) for the transmitter at the origin is at a distance $R$ and is not a part of the process $\Phi$. Conditioning the existence of a point at the origin leads to a cluster around the origin (denoted by a bold circle). The cluster at the origin need not be centered around the origin.

given by

$$
I_{\Phi}(z)=\sum_{x \in \Phi \backslash\{o\}} h_{\times} \ell(x-z) .
$$

We focus on Matern cluster processes and Thomas cluster processes, which belong to the class of the Neyman-Scott cluster processes. The cluster process consists of a parent $\operatorname{PPP} \Phi_{p}=\left\{\mathrm{x}_{1}, \mathrm{x}_{2}, \ldots\right\}$ of density $\lambda_{p}$. The clusters are of the form $N_{\mathrm{x}_{i}}=N_{i}+\mathrm{x}_{i}$ for each $\mathrm{x}_{i} \in \Phi_{p}$. The $N_{i}$ are a family of identical, independently distributed point sets, and also independent of the parent process. Since all the clusters are identically distributed, we can talk about a representative cluster with the same distribution as that of the clusters of the process. This representative cluster is denoted by $N_{o}$.

In the Matern and Thomas cluster processes, the number of points in the representative cluster is Poisson distributed with mean $\bar{c}$. The 
daughter points of the representative cluster $N_{o}$ are scattered independently and with a identical spatial distribution

$$
F_{\mathrm{cl}}(A)=\int_{A} f_{\mathrm{cl}}(x) \mathrm{d} x, \quad A \subset \mathbb{R}^{2},
$$

around the origin. For the Matern cluster process each point is uniformly distributed in a ball of radius $a$ around the origin. So the density function $f_{\mathrm{cl}}(x)$ is given by

$$
f_{\mathrm{cl}}(x)= \begin{cases}\frac{1}{\pi a^{2}}, & \|x\| \leq a \\ 0 & \text { otherwise. }\end{cases}
$$

In the Thomas cluster process, each point is scattered using a symmetric normal distribution with variance $\sigma^{2}$ around the origin. So the density function $f_{\mathrm{cl}}(x)$ is given by

$$
f_{\mathrm{cl}}(x)=\frac{1}{2 \pi \sigma^{2}} \exp \left(-\frac{\|x\|^{2}}{2 \sigma^{2}}\right) .
$$

The complete process $\Phi$ is given by

$$
\Phi=\bigcup_{\mathrm{x} \in \Phi_{p}} N_{\mathrm{x}}
$$

and the PGFL is (see (A.4))

$$
\begin{aligned}
\mathcal{G}[\nu] & \triangleq \mathbb{E}\left[\prod_{\mathrm{x} \in \Phi} \nu(\mathrm{x})\right] \\
& =\exp \left(-\lambda_{p} \int_{\mathbb{R}^{d}} 1-M\left(\int_{\mathbb{R}^{d}} \nu(x+y) f_{\mathrm{cl}}(x) \mathrm{d} x\right)\right),
\end{aligned}
$$

where $M(z)=\exp (\bar{c}(z-1))$. The second-order product density of the Matern and the Thomas cluster processes [45, Section 5.3] is

$$
\rho^{(2)}(z)=\lambda_{p} \bar{c}^{2}\left[\left(f_{\mathrm{cl}} * f_{\mathrm{cl}}\right)(z)+\lambda_{p}\right] .
$$

See Section A.1.3 and [45, Section 5.3] for a detailed description of clustered point processes. 


\subsection{Interference Characterization}

In this section, we will derive the statistical characteristics of the interference when the transmitters are distributed as a PCP. As shall be evident in the next section, we will derive the interference properties conditioning on a transmitter being at the origin (the desired transmitter). Differently from the PPP, placing an additional transmitter at the origin (even though it does not contribute to the interference) makes the distribution of the interference non-stationary, i.e., the interference $I_{\Phi}(z)$ distribution depends on $z$. The average interference (conditioned on the event that there is a point of the process at the origin) is

$$
\begin{aligned}
\mathbb{E}^{! o}\left[I_{\Phi}(z)\right] & =\mathbb{E}^{! o}\left[\sum_{\mathrm{x} \in \Phi} h_{\mathrm{x}} \ell(\mathrm{x}-z)\right] \\
& =\mathbb{E}[h] \lambda \int_{\mathbb{R}^{2}} \ell(x-z) \mathcal{K}_{2}(\mathrm{~d} x),
\end{aligned}
$$

where $\mathcal{K}_{2}$ is the second-order moment measure (A.8) and $\mathbb{E}^{! o}$ is the expectation with respect to the reduced Palm measure. Equivalently, by the relation (A.9), we have

$$
\mathbb{E}^{! o}\left[I_{\Phi}(z)\right]=\frac{\mathbb{E}[h]}{\lambda} \int_{\mathbb{R}^{2}} \ell(x-z) \rho^{(2)}(x) \mathrm{d} x .
$$

Example: Thomas Cluster Process. In this case, from [45, p. 160],

$$
\frac{\rho^{(2)}(x)}{\lambda^{2}}=1+\frac{1}{4 \pi \lambda_{p} \sigma^{2}} \exp \left(\frac{-\|x\|^{2}}{4 \sigma^{2}}\right),
$$

where $\lambda=\lambda_{p} \bar{c}$. We obtain

$$
\mathbb{E}^{! o}\left[I_{\Phi}(z)\right]=\mathbb{E} I_{\text {Poi }(\lambda)}+\frac{\bar{c} \mathbb{E}[h]}{4 \pi \sigma^{2}} \int_{\mathbb{R}^{2}} \ell(x-z) \exp \left(\frac{-\|x\|^{2}}{4 \sigma^{2}}\right) \mathrm{d} x .
$$

$\mathbb{E} I_{\text {Poi }(\lambda)}$ is the average interference when the nodes are distributed as a PPP with intensity $\lambda . \mathbb{E} I_{\mathrm{Poi}(\lambda)}$ is finite only when $\ell(x)$ is bounded at the origin. The above expression also shows that the mean interference is indeed larger than for the PPP, and that $\mathbb{E}^{! o}\left[I_{\Phi}(z)\right] \rightarrow \mathbb{E} I_{\mathrm{Poi}}$ when $\|z\| \rightarrow \infty$, which is intuitive since the effect of the cluster at the origin vanishes as $\|z\|$ increases. We now derive the conditional Laplace transform of the interference. First we show that it can be expressed using the conditional PGFL of $\Phi$. 
Lemma 4.1. The conditional Laplace transform of the interference at $z$ can be expressed as

$$
\mathcal{L}_{I_{\Phi}(z)}^{! o}(s)=\tilde{\mathcal{G}}\left[\mathcal{L}_{h}(s \ell(\cdot-z))\right],
$$

where $\tilde{\mathcal{G}}$ is the conditional $\mathrm{PGFL}^{2}$ (A.13) of the transmitter point process $\Phi$.

Proof. We have

$$
\begin{aligned}
\mathcal{L}_{I_{\Phi}(z)}^{! o}(s) & =\mathbb{E}^{! o} \exp \left(-s \sum_{\mathrm{x} \in \Phi} h_{\mathrm{x}} \ell(\mathrm{x}-z)\right) \\
& =\mathbb{E}^{! o}\left[\prod_{\mathrm{x} \in \Phi} \exp \left(-s h_{\mathrm{x}} \ell(\mathrm{x}-z)\right)\right] \\
& \stackrel{(a)}{=} \mathbb{E}^{! o}\left[\prod_{\mathrm{x} \in \Phi} \mathcal{L}_{h}(s \ell(\mathrm{x}-z))\right],
\end{aligned}
$$

where $(a)$ follows from the independence of $h_{x}$ and the result follows from the definition of the conditional PGFL (A.13).

Since the reduced Palm measure of the PCP is not stationary [45, p. 158], it follows from Lemma 4.1 that the interference distribution depends on the location $z$. We now derive the conditional PGFL of the Matern and the Thomas PCPs, which is required to obtain the conditional Laplace transform of the interference. The basic tool for the proof is the description of the reduced Palm measure in Theorem A.6. In a loose sense, Theorem A.6 indicates that conditioning the existence of a node at the origin is "almost" like placing an independent cluster at the origin. We also use the fact that each cluster of the Matern or Thomas is a non-stationary Poisson point process with intensity $\lambda(x)=\bar{c} f_{\text {cl }}(x)$ (since the number of nodes in each cluster is a Poisson random variable with mean $\bar{c}$ ) and hence Slivnyak's Theorem A.5 can be applied to an individual cluster.

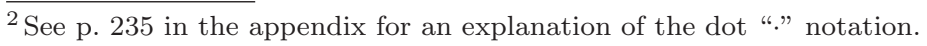


Theorem 4.2. Let $0 \leq v(x) \leq 1$. The conditional probability generating functional of the Thomas and Matern cluster processes is

$$
\tilde{\mathcal{G}}[v]=\mathcal{G}[v] \int_{\mathbb{R}^{2}} G_{\mathrm{cl}}[v(\cdot-y)] f_{\mathrm{cl}}(y) \mathrm{d} y
$$

where

$$
G_{\mathrm{cl}}[v]=\exp \left(-\bar{c}\left[1-\int_{\mathbb{R}^{2}} v(x) f_{\mathrm{cl}}(x) \mathrm{d} x\right]\right)
$$

is the PGFL of the representative cluster.

Proof. Let $Y_{x}=Y+x$. From Theorem A.6, we have

$$
\tilde{\Omega}^{! o}(Y)=\frac{1}{\bar{c}} \mathbb{E}\left(\sum_{\mathrm{x} \in N_{o}} \mathbf{1}_{Y_{\mathrm{x}}}\left(N_{o} \backslash\{\mathrm{x}\}\right)\right) .
$$

Let $\Omega()$ denote the probability distribution of the representative cluster. Using the Campbell-Mecke theorem [45, p. 119], we get

$$
\begin{aligned}
\tilde{\Omega}^{! o}(Y) & =\frac{1}{\bar{c}} \int_{\mathbb{R}^{2}} \int_{\mathrm{N}} \mathbf{1}_{Y_{x}}\left(N_{o}\right) \Omega^{! x}\left(\mathrm{~d} N_{o}\right) \bar{c} F_{\mathrm{cl}}(\mathrm{d} x) \\
& =\int_{\mathbb{R}^{2}} \int_{\mathrm{N}} \mathbf{1}_{Y_{x}}\left(N_{o}\right) \Omega^{! x}\left(\mathrm{~d} N_{o}\right) f_{\mathrm{cl}}(x) \mathrm{d} x,
\end{aligned}
$$

where $\mathrm{N}$ denotes the set of finite and simple sequences on $\mathbb{R}^{2}$ (see p. 227 in the appendix).

Since the representative cluster is a PPP, by Slivnyak's theorem we have $\Omega^{! x}()=.\Omega($.$) . Hence$

$$
\begin{aligned}
\tilde{\Omega}^{! o}(Y) & =\int_{\mathbb{R}^{2}} \int_{\mathrm{N}} \mathbf{1}_{Y_{x}}\left(N_{o}\right) \Omega\left(\mathrm{d} N_{o}\right) f_{\mathrm{cl}}(x) \mathrm{d} x \\
& =\int_{\mathbb{R}^{2}} \Omega\left(Y_{x}\right) f_{\mathrm{cl}}(x) \mathrm{d} x .
\end{aligned}
$$


For notational convenience let $\psi$ denote $N_{o}$ and let $\psi_{y}=\psi+y$. Using Theorem A.5, we have

$$
\begin{aligned}
\tilde{\mathcal{G}}[v] & =\int_{\mathrm{N}} \int_{\mathrm{N}_{x \in \phi \cup \psi}} \prod v(x) \mathbf{P}(\mathrm{d} \phi) \tilde{\Omega}^{! o}(\mathrm{~d} \psi) \\
& =\int_{\mathrm{N}} \prod_{\mathrm{x} \in \phi} v(x) \mathbf{P}(\mathrm{d} \phi) \int_{\mathrm{N}} \prod_{x \in \psi} v(x) \tilde{\Omega}^{! o}(\mathrm{~d} \psi) \\
& =\mathcal{G}[v] \int_{\mathrm{N}} \prod_{x \in \psi} v(x) \tilde{\Omega}^{! o}(\mathrm{~d} \psi) \\
& \stackrel{(a)}{=} \mathcal{G}[v] \int_{\mathbb{N}} \prod_{x \in \psi} v(x) \int_{\mathbb{R}^{2}} \Omega\left(\mathrm{d} \psi_{y}\right) f_{\mathrm{cl}}(y) \mathrm{d} y \\
& =\mathcal{G}[v] \int_{\mathbb{R}^{2}} \int_{\mathrm{N}} \prod_{x \in \psi} v(x) \Omega\left(\mathrm{d} \psi_{y}\right) f_{\mathrm{cl}}(y) \mathrm{d} y \\
& =\mathcal{G}[v] \int_{\mathbb{R}^{2}} \int_{\mathrm{N}} \prod_{x \in \psi} v(x-y) \Omega(\mathrm{d} \psi) f_{\mathrm{cl}}(y) \mathrm{d} y \\
& \stackrel{(b)}{=} \mathcal{G}[v] \int_{\mathbb{R}^{2}} G_{\mathrm{cl}}[v(\cdot-y)] f_{\mathrm{cl}}(y) \mathrm{d} y .
\end{aligned}
$$

(a) follows from (4.10), and (b) follows from the definition of $\mathcal{G}($.$) .$

The above result can be interpreted as follows: The Palm measure of the clustered process is the independent superposition of the original process and a cluster at the origin which is randomly translated by an amount drawn from the density $f_{\mathrm{cl}}(y)$. Hence the resultant conditional PGFL is the product of the PGFL of the clustered process and the PGFL of the representative cluster shifted by $y$ and averaged by the density $f_{\mathrm{cl}}(y)$. In Matern and Thomas cluster processes, the number of nodes in each cluster is a Poisson random variable with mean $\bar{c}$. If instead, each cluster has a fixed number of nodes $\bar{c} \in \mathbb{Z}^{+}$, it can be shown that the conditional PGFL is

$$
\tilde{\mathcal{G}}[v]=\hat{\mathcal{G}}[v] \int_{\mathbb{R}^{2}}\left(\int_{\mathbb{R}^{2}} v(x-y) f_{\mathrm{cl}}(x) \mathrm{d} x\right)^{\bar{c}-1} f_{\mathrm{cl}}(y) \mathrm{d} y,
$$


where

$$
\hat{\mathcal{G}}[v]=\exp \left(-\lambda_{p} \int_{\mathbb{R}^{2}} 1-\left(\int_{\mathbb{R}^{2}} v(x+y) f_{\mathrm{cl}}(y) \mathrm{d} y\right)^{\bar{c}} \mathrm{~d} x\right) .
$$

We observe that when $\bar{c}=1$ we get back the PGFL of the PPP.

The conditional Laplace transform of the interference follows from Lemma 4.1 and Theorem 4.2. It can be used to derive the moments of the interference. For example the average interference is equal to $\mathbb{E}^{! o}\left[I_{\Phi}(z)\right]=-\left.\frac{\mathrm{d}}{\mathrm{d} s} \mathcal{L}_{I_{\Phi}(z)}^{!_{0}}(s)\right|_{s=0}$. When $\ell(x)=\|x\|^{-\alpha}$, the conditional Laplace transform can be used to prove that the interference has a heavy tail. The following lemma provides the asymptotics of the tail.

Lemma 4.3. For $\ell(x)=\|x\|^{-\alpha}$, the lower and upper bounds of the CCDF $\bar{F}_{I}(y)$ of the interference at location $z$, when the nodes are distributed as a Neyman-Scott cluster process, scale as follows for $y \rightarrow \infty$ :

$$
\begin{aligned}
\bar{F}_{I}^{l}(y) & \sim C_{z} y^{-\delta} \\
\bar{F}_{I}^{u}(y) & \sim \frac{C_{z}}{1-\delta} y^{-\delta},
\end{aligned}
$$

where $C_{z}=\pi \bar{c}\left[\left(f_{\mathrm{cl}} * f_{\mathrm{cl}}\right)(z)+\lambda_{p}\right] \mathbb{E}\left[h^{\delta}\right]$ and $\delta=2 / \alpha$.

This lemma is proved in more generality in Section 5. For a proof specifically for the cluster process, see [13, Lemma 3]. From this lemma we see that the interference does not have any finite integer moments when $\ell(x)=\|x\|^{-\alpha}$. With (4.3), $C_{z}$ can also be expressed as

$$
C_{z}=\frac{\pi \rho^{(2)}(z)}{\lambda} \mathbb{E}\left[h^{\delta}\right]
$$

For Rayleigh fading and the Thomas cluster process we have

$$
C_{z}=\pi \lambda_{p} \bar{c} \Gamma(1+\delta)\left[1+\frac{1}{4 \pi \lambda_{p} \sigma^{2}} \exp \left(\frac{-\|z\|^{2}}{4 \sigma^{2}}\right)\right],
$$

and when $\Phi$ is a Matern cluster process

$$
C_{z}= \begin{cases}\lambda_{p} \bar{c} \Gamma(1+\delta)\left[1+\frac{4\|z\|}{\pi a^{2}}\left(\cos ^{-1}\left(a_{z}\right)-a_{z} \sqrt{1-a_{z}^{2}}\right)\right] & a_{z}<1 \\ \lambda_{p} \bar{c} \Gamma(1+\delta) & a_{z} \geq 1\end{cases}
$$




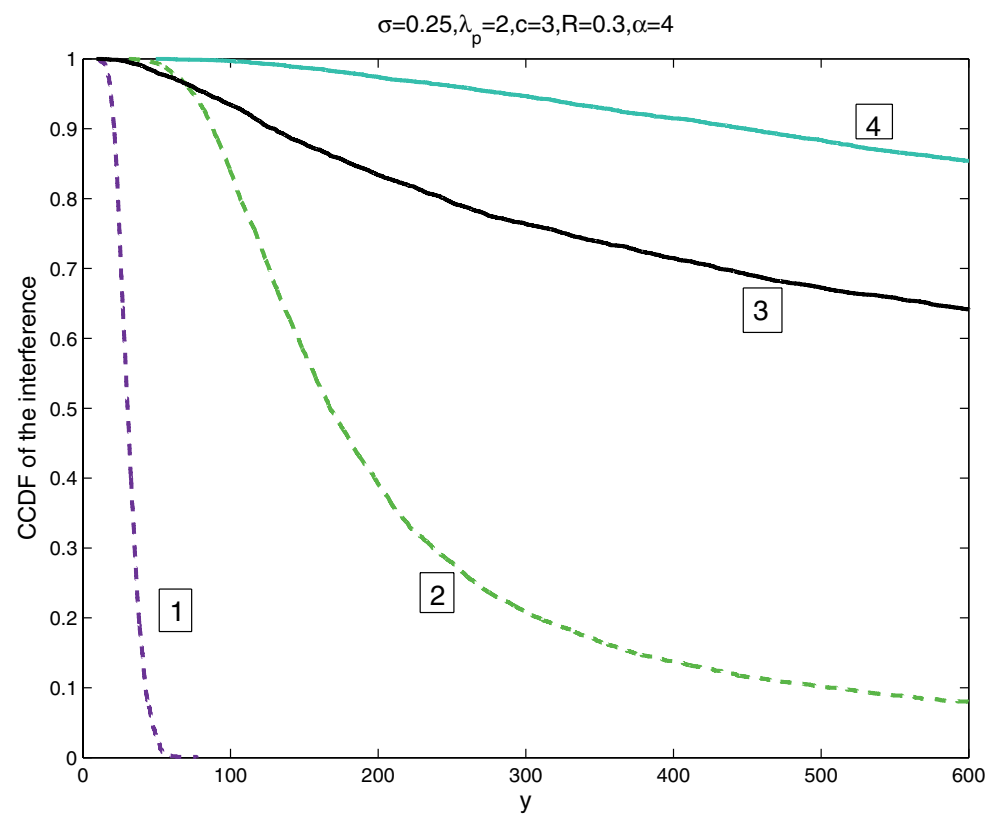

Fig. 4.3 Comparison of the interference CCDF for a Thomas cluster process for different path loss models and different fading statistics. The common parameters are $\lambda_{p}=2, \bar{c}=3$, $\sigma=0.25, \alpha=4$, and $\|z\|=0.3$. The curves were obtained by Monte-Carlo simulation (from [13] (C) 2009 IEEE).

where $a_{z}=\|z\| /(2 a)$. In Theorem 5.6, we prove that $\mathbb{P}\left(I_{\Phi}(z) \geq y\right) \sim$ $\pi \rho^{(2)}(z) \lambda^{-1} \mathbb{E}\left[h^{\delta}\right] y^{-\delta}$ and hence it can be concluded that the lower bound is closer to the actual CCDF for large $y$.

When $\ell(x)$ is bounded at the origin, the interference tail follows the distribution of the fading. In Figure 4.3 curves \#1 and \#2 correspond to $\ell(x)=\left(1+\|x\|^{\alpha}\right)^{-1}$. Curve \#1 corresponds to Rayleigh fading and exhibits an exponential decay. Curve \#2 for which $h$ is generalized Pareto $^{3}$ with parameters $k=1, \theta=0, \sigma_{p}=1$ (a hypothetical fading distribution which exhibits power law decay) exhibits a power law decay. Curves \#3 (generalized Pareto) and \#4 (Rayleigh) correspond to $\ell(x)=\|x\|^{-\alpha}$ and exhibit a heavy tail for both fading distributions.

${ }^{3}$ The PDF of the generalized Pareto distribution is $f(x)=\sigma^{-1}\left(1+k \frac{x-\theta}{\sigma}\right)^{-1-1 / k}$. 
We can also use the conditional Laplace transform to obtain the nearest-neighbor distribution as $N(r)=\mathcal{G}\left[\mathbf{1}_{b(o, r)^{c}}(x)\right]$. All the reduced Palm moments of the PCP can be derived from the conditional PGFL obtained before.

\subsection{Outage Analysis}

The probability of success for the transmitter located at the origin and its receiver at $z$ is

$$
\begin{aligned}
p_{s}(\theta) & =\mathbb{P}\left(\frac{h \ell(z)}{W+I_{\Phi \backslash\{o\}}(z)} \geq \theta \mid \text { transmitter at the origin }\right) \\
& =\mathbb{P}^{! o}\left(\frac{h \ell(z)}{W+I_{\Phi}(z)} \geq \theta\right) .
\end{aligned}
$$

For Rayleigh fading,

$$
\begin{aligned}
p_{s}(\theta) & =\int_{0}^{\infty} e^{-s \theta / \ell(z)} \mathrm{d} \mathbb{P}^{! o}\left(W+I_{\Phi}(z) \leq s\right) \\
& =\mathcal{L}_{I_{\Phi}(z)}^{! o}(\theta / \ell(z)) \mathcal{L}_{W}(\theta / \ell(z)) .
\end{aligned}
$$

$\mathcal{L}_{I_{\Phi}(z)}^{! o}$ is the conditional Laplace transform of the interference and $\mathcal{L}_{W}$ denotes the Laplace transform of the noise. We now use the conditional PGFL of PCP and (4.13) to evaluate the success probability.

Theorem 4.4 (Success probability). The probability of successful transmission between the transmitter at the origin and the receiver located at $z \in \mathbb{R}^{2}$, when $W \equiv 0$ (no noise), is

$$
\begin{aligned}
P_{s}(\theta)= & \underbrace{\exp \left\{-\lambda_{p} \int_{\mathbb{R}^{2}}[1-\exp (-\bar{c} \beta(z, y))] \mathrm{d} y\right\}}_{T_{1}} \\
& \times \underbrace{\int_{\mathbb{R}^{2}} \exp (-\bar{c} \beta(z, y)) f_{\mathrm{cl}}(y) \mathrm{d} y}_{T_{2}}
\end{aligned}
$$

where

$$
\beta(z, y)=\int_{\mathbb{R}^{2}} \frac{\ell(x-y-z)}{\frac{\ell(z)}{\theta}+\ell(x-y-z)} f_{\mathrm{cl}}(x) \mathrm{d} x .
$$


Proof. From Lemma 4.1, we have

$$
\begin{aligned}
\mathcal{L}_{I_{\Phi}(z)}^{! o}\left(\frac{\theta}{\ell(z)}\right) & =\tilde{\mathcal{G}}\left[\mathcal{L}_{h}\left(\frac{\theta}{\ell(z)} \ell(\cdot-z)\right)\right] \\
& \stackrel{(a)}{=} \tilde{\mathcal{G}}\left[\frac{1}{1+\frac{\theta}{\ell(z)} \ell(\cdot-z)}\right],
\end{aligned}
$$

where $(a)$ follows from the Laplace transform of the exponentially distributed $h$. Hence the result follows from Theorem 4.2.

Remarks. The interference can be written as a sum of two independent terms, one being the interference caused at the receiver by the transmitter's own cluster and the other being the interference caused by other clusters, i.e.,

$$
I_{\Phi}(z)=I_{\Phi \backslash \text { Tx-cluster }}(z)+I_{\text {Tx-cluster }}(z) .
$$

Since we are considering a Poisson cluster process, these two terms are independent. The contribution of the interference in the success probability is $\mathcal{L}_{I_{\Phi}(z)}^{! o}(\theta / \ell(z))$. Since the Laplace transform of the sum of independent random variables is the product of the individual Laplace transforms, we have the product of two terms in Theorem 4.4. The term $T_{1}$ in (4.14) captures the interference without the cluster at the origin (i.e., without conditioning); it is independent of the position $z$ since the original cluster process is stationary, which can be verified by a change of variables $y_{1}=y+z$. The second term $T_{2}$ is the contribution of the transmitter's cluster; it is identical for all $z$ with $\|z\|=R$ since $f_{\mathrm{cl}}$ and $\ell$ are isotropic. So the success probability itself is the same for all $z$ at distance $R$. This is because the Palm distribution is always isotropic when the original distribution is motion-invariant $[45,4.4 .8]$.

From the above argument we observe that $p_{s}(\theta)$ depends only on $\|z\|=R$ and not on the angle of $z$. So the success probability should be interpreted as an average over the circle $\|z\|=R$, i.e., the receiver may be uniformly located anywhere on the circle of radius $R$ around the origin.

Intuitively for the same intensity of transmitting nodes, a Poisson cluster process will have bigger vacancies (areas without any transmitters) than a PPP. Hence for $R$ larger than a critical radius that is a 


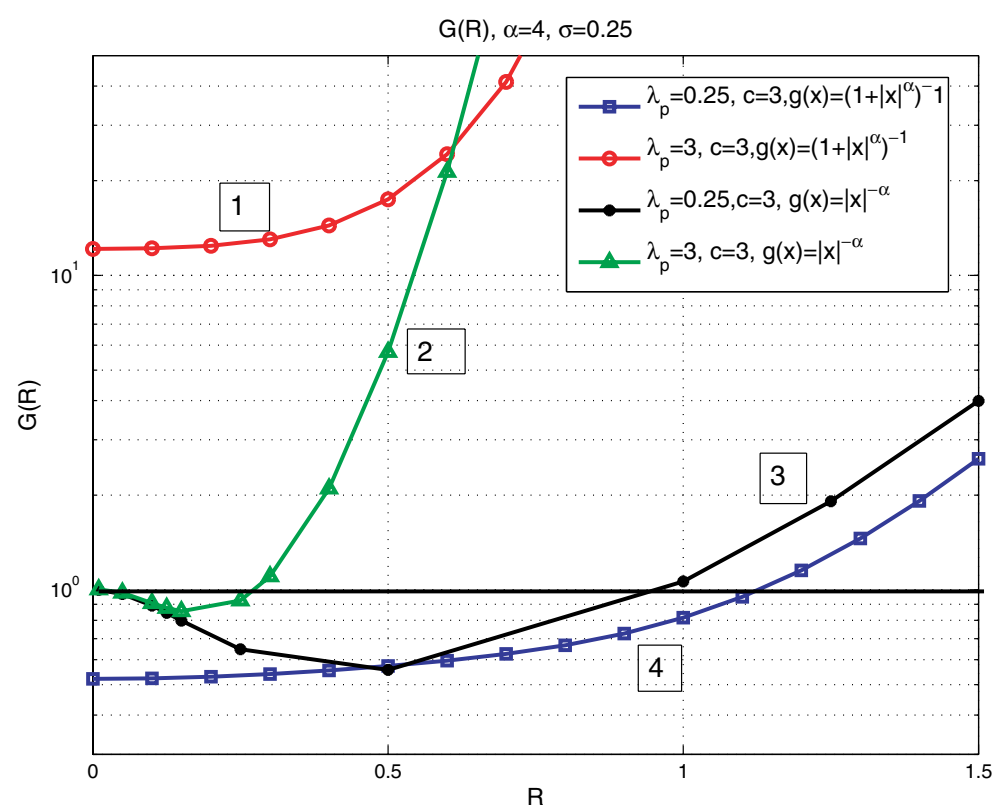

Fig. $4.4 G(R)$ versus $R, \alpha=4, \sigma=0.25$. Observe that the gain curves \#2 and \#3, which correspond to the singular channel, start at 1 , decrease and then increase above unity. The crossover point $R^{*}$ is approximately $1 /\left(2 \sqrt{\lambda_{p}}\right)$, which is the average nearest-neighbor distance of the parent process. Curves \#1 and \#4 correspond to a bounded path loss model, and the crossover point $R^{*}$ may not exist (from [13] (C) 2009 IEEE).

function of $\sigma^{2}$ or $a, \lambda_{p}, \bar{c}$ and, the probability that an interfering transmitter is close to the receiver is smaller for a cluster process than a PPP. For a smaller $R$, the receiver is in the cluster, which causes more outages. Let the clustering gain be defined as

$$
G(R) \triangleq \frac{p_{s}^{\mathrm{cl}}}{p_{s}^{\mathrm{ppp}}}
$$

where $p_{s}^{\text {cl }}$ represents the success probability when the transmitters are clustered and $p_{s}^{\mathrm{ppp}}$ represent the same for a PPP. We can then show that when $\ell(x)=\|x\|^{-\alpha}, G(R)<1$ for small $R$ and $G(R)>1$ for large $R$, i.e., that there exists a critical link distance $R^{*}$ above which clustering is beneficial. For a bounded path loss function, the crossover point may not exist and $G(R)$ may be greater than unity for all $R$. See Figure 4.4. A detailed discussion of the clustering gain is provided in [13]. 
5

\section{Interference in General Motion-Invariant Networks}

\subsection{System Model}

In this section, we consider transmitters distributed in a stationary and isotropic fashion on the plane. Stationary and isotropic point processes are also called motion-invariant. Please refer to the appendix for a mathematical description of such point processes. We give four examples:

Homogeneous Poisson point process: A PPP of constant density $\lambda$ is a stationary and isotropic point process and hence motion-invariant. Also any point process with finite number of points in the plane cannot be stationary and hence cannot be motion-invariant.

Poisson cluster process: Poisson cluster processes, defined in the appendix and analyzed in Section 4, are motion-invariant.

Shifted lattice process: This point process $\Phi$ is the randomly translated and rotated square lattice. More precisely,

$$
\Phi=\sqrt{\lambda} \mathbb{Z}^{2} e^{j \beta}+U
$$

where $\beta$ is uniformly distributed in $[0,2 \pi]$ and $U$ is uniformly distributed in $[0, \sqrt{\lambda}]$. 
Hole-1 process: Consider two stationary PPPs $\Phi_{s}$ and $\Phi_{p}$ of intensities $\lambda_{s}$ and $\lambda_{p}$ with $\lambda_{p}<\lambda_{s}$. The lower intensity process $\Phi_{p}$ models the primary users and $\Phi_{s}$ the secondary users in a cognitive network. A secondary user $\mathrm{x} \in \Phi_{s}$ with no primary user in a disk of radius $a$ around itself is allowed to transmit. More formally,

$$
\Phi_{s}^{t}=\left\{\mathrm{x} \in \Phi_{s}: b(\mathrm{x}, a) \cap \Phi_{p}=\emptyset\right\} .
$$

The average number of secondary transmitters in a set $B$ is

$$
\begin{aligned}
\mathbb{E} \Phi_{s}^{t}(B) & =\mathbb{E} \sum_{\mathrm{x} \in \Phi_{s} \cap B} \prod_{\mathrm{y} \in \Phi_{p}}\left(1-\mathbf{1}_{b(\mathrm{x}, a)}(\mathrm{y})\right) \\
& \stackrel{(a)}{=} \mathbb{E} \sum_{\mathrm{x} \in \Phi_{s} \cap B} \mathbb{E} \prod_{\mathrm{y} \in \Phi_{p}}\left(1-\mathbf{1}_{b(\mathrm{x}, a)}(\mathrm{y})\right) \\
& \stackrel{(b)}{=} \mathbb{E} \sum_{\mathrm{x} \in \Phi_{s} \cap B} \exp \left(-\lambda_{p} \int_{\mathbb{R}^{2}} \mathbf{1}_{b(\mathrm{x}, a)}(y) \mathrm{d} y\right) \\
& \stackrel{(c)}{=} \lambda_{s} \exp \left(-\lambda_{p} \pi a^{2}\right)|B|,
\end{aligned}
$$

where $(a)$ follows from the independence of the two point process, $(b)$ follows from the PGFL of $\Phi_{p}$, and (c) follows from the Campbell theorem applied to $\Phi_{s}$. From the above, we observe that the density of $\Phi_{s}^{t}$ is $\lambda_{s} \exp \left(-\lambda_{p} \pi a^{2}\right)$.

Every transmitter is assumed to transmit with unit power. The intended transmitter is located at the origin and is a part of the transmit point process $\Phi$. The interference observed at $z \in \mathbb{R}^{2}$ is

$$
I_{\Phi}(z)=\sum_{\mathrm{x} \in \Phi \backslash\{o\}} h_{\mathrm{x}} \ell(\mathrm{x}-z),
$$

where the fading process $h_{\mathrm{x}}$ is iid with $\mathrm{CDF} F_{h}, \mathrm{PDF} f_{h}$, and unit mean. In this section we provide the characterization of the (complementary) CDF of $I_{\Phi}(z)$ given that there is a transmitting node at the origin. So all probabilities are conditioned on the event that there is a transmitting node at the origin, i.e., we are using Palm probabilities [8, 29, 45]. Let $\tilde{\mathcal{G}}[v]$ denote the conditional probability generating functional of the point process $\Phi$, i.e.,

$$
\tilde{\mathcal{G}}[v]=\mathbb{E}^{! o}\left[\prod_{\mathrm{x} \in \Phi} v(\mathrm{x})\right],
$$


where $v: \mathbb{R}^{2} \rightarrow[0, \infty)$ is a well-behaved function [8].

In this section, we will also consider the effect of the path loss function $\ell(x)$ on the distribution of the interference. We consider the following path loss models:

(1) Singular model: $\ell(x)=\|x\|^{-\alpha}, \alpha>2$.

(2) Non-singular (bounded) model: $\ell(x)=\left(1+\|x\|^{\alpha}\right)^{-1}, \alpha>2$.

We require $\alpha>2$ since we want $\int_{b(o, 1)^{c}} l(x)<\infty$, i.e., the path loss model should be well-behaved except possibly at the origin. In the literature [11], an exponential path loss model i.e., $\ell(x)=\exp (-\gamma\|x\|) /\|x\|^{\alpha}$ is also used. But from the interference perspective, it is similar to the singular path loss model. As before, we will use $\delta$ to denote $2 / \alpha$.

\subsection{Properties of the Interference}

We first start by deriving the first and the second conditional moments of the interference.

Lemma 5.1. The mean interference is

$$
\mathbb{E}^{! o}\left[I_{\Phi}(z)\right]=\frac{1}{\lambda} \int_{\mathbb{R}^{2}} \ell(x-z) \rho^{(2)}(x) \mathrm{d} x .
$$

Proof.

$$
\begin{aligned}
\mathbb{E}^{! o}\left[I_{\Phi}(z)\right] & =\mathbb{E}^{! o} \sum_{\mathrm{x} \in \Phi} h_{x} \ell(\mathrm{x}-z) \\
& \stackrel{(a)}{=} \mathbb{E}[h] \lambda \int_{\mathbb{R}^{2}} \ell(x-z) \mathcal{K}_{2}(\mathrm{~d} x) \\
& \stackrel{(b)}{=} \frac{1}{\lambda} \int_{\mathbb{R}^{2}} \ell(x-z) \rho^{(2)}(x) \mathrm{d} x .
\end{aligned}
$$

(a) follows from the definition of the $n$-th factorial moment measure, see (A.10) and (b) from the definition of $\rho^{(2)}(x)$.

The average interference is finite for the non-singular path loss model. For $\ell(x)=\|x\|^{-\alpha}$, the average interference is finite if and only if $\rho^{(2)}(x)$ 
is zero in a small neighborhood of $z$. From the definition of $\rho^{(2)}(x)$ this implies that there should not be any interfering transmitter close to the receiver located at $z$. For a PPP and $\operatorname{PCP} \rho^{(2)}(z) \neq 0$ for $z>0$ and hence the average interference is infinite. For the shifted lattice process, $\rho^{(2)}(x)=0$ for $\|x\|<\sqrt{\lambda}$ and hence the average interference is finite for $z<\sqrt{\lambda}$.

Lemma 5.2. The second moment of the interference is

$$
\begin{aligned}
\mathbb{E}^{! o}\left[I_{\Phi}^{2}(z)\right]= & \frac{\mathbb{E}\left[h^{2}\right]}{\lambda} \int_{\mathbb{R}^{2}} \ell^{2}(x-z) \rho^{(2)}(x) \mathrm{d} x \\
& +\frac{\mathbb{E}[h]^{2}}{\lambda} \int_{\mathbb{R}^{2}} \int_{\mathbb{R}^{2}} \ell\left(x_{1}-z\right) \ell\left(x_{2}-z\right) \rho^{(3)}\left(x_{1}, x_{2}\right) \mathrm{d} x_{1} \mathrm{~d} x_{2} .
\end{aligned}
$$

Proof. We have

$$
\begin{aligned}
\mathbb{E}^{! o} & {\left[I_{\Phi}^{2}(z)\right] } \\
& =\mathbb{E}^{! o}\left[\sum_{\mathrm{x} \in \Phi} h_{x} \ell(\mathrm{x}-z)\right]^{2} \\
& =\mathbb{E}^{! o}\left[\sum_{\mathrm{x} \in \Phi} h_{\mathrm{x}}^{2} \ell^{2}(\mathrm{x}-z)\right]+\mathbb{E}^{! o}\left[\sum_{\mathrm{x}_{1}, \mathrm{x}_{2} \in \Phi}^{\neq} h_{\mathrm{x}_{1}} h_{\mathrm{x}_{2}} \ell\left(\mathrm{x}_{1}-z\right) \ell\left(\mathrm{x}_{2}-z\right)\right] \\
& \stackrel{(a)}{=} \mathbb{E}\left[h^{2}\right] \mathbb{E}^{! o}\left[\sum_{\mathrm{x} \in \Phi} \ell^{2}(\mathrm{x}-z)\right]+\mathbb{E}[h]^{2} \mathbb{E}^{! o}\left[\sum_{\mathrm{x}_{1}, \mathrm{x}_{2} \in \Phi}^{\neq} \ell\left(\mathrm{x}_{1}-z\right) \ell\left(\mathrm{x}_{2}-z\right)\right]
\end{aligned}
$$

where $\sum^{\neq}$indicates that the summation is to be taken over all pairs of distinct points in $\Phi$. (a) follows from the independence of the fading. For the first term,

$$
\begin{aligned}
\mathbb{E}^{! o}\left[\sum_{\mathrm{x} \in \Phi} \ell^{2}(\mathrm{x}-z)\right] & \stackrel{(a)}{=} \lambda \int_{\mathbb{R}^{2}} \ell^{2}(x-z) \mathcal{K}_{2}(\mathrm{~d} x) \\
& \stackrel{(b)}{=} \frac{1}{\lambda} \int_{\mathbb{R}^{2}} \ell^{2}(x-z) \rho^{(2)}(x) \mathrm{d} x .
\end{aligned}
$$


Similarly, for the second term,

$$
\begin{aligned}
\mathbb{E}^{! o}\left[\sum_{\mathrm{x}_{1}, \mathrm{x}_{2} \in \Phi}^{\neq} \ell\left(\mathrm{x}_{1}-z\right) \ell\left(\mathrm{x}_{2}-z\right)\right] \\
\stackrel{(c)}{=} \lambda^{2} \int_{\mathbb{R}^{2}} \int_{\mathbb{R}^{2}} \ell\left(x_{1}-z\right) \ell\left(x_{2}-z\right) \mathcal{K}_{3}\left(\mathrm{~d} x_{1} \times \mathrm{d} x_{2}\right) \\
\stackrel{(d)}{=} \frac{1}{\lambda} \int_{\mathbb{R}^{2}} \int_{\mathbb{R}^{2}} \ell\left(x_{1}-z\right) \ell\left(x_{2}-z\right) \rho^{(3)}\left(x_{1}, x_{2}\right) \mathrm{d} x_{1} \mathrm{~d} x_{2} .
\end{aligned}
$$

$(a),(b),(c)$, and $(d)$ follow from the definition of the $n$-th order product density in the appendix.

When $\Phi$ is PPP, we have $\rho^{(2)}(x)=\lambda^{2}$ and $\rho^{(3)}(x)=\lambda^{3}$ and hence

$$
\mathbb{E}^{! o}\left[I_{\Phi}^{2}(z)\right]=\mathbb{E}\left[h^{2}\right] \lambda \int_{\mathbb{R}^{2}} \ell^{2}(x) \mathrm{d} x+\mathbb{E}[h]^{2} \lambda^{2}\left(\int_{\mathbb{R}^{2}} \ell(x) \mathrm{d} x\right)^{2},
$$

in agreement with (3.52). We observe that the interference moments and hence the distribution depend on the location $z$ for a general point process. The distribution of the $I_{\Phi}(z)$ does not depend on the direction of $z$ because of the isotropy of the Palm distribution for motioninvariant processes.

\subsection{Bounds on the Interference Distribution}

In the previous sections, we have derived the Laplace transform of the interference for the PPP and the PCP. Although in theory, the Laplace transform provides the complete description of the interference, it is more beneficial to know the CDF or the PDF of the interference explicitly. In this section we provide bounds on the CCDF of the interference when the transmitting nodes are homogeneously distributed on the plane.

The basic idea behind the proof is easy to understand when fading is absent, $\ell(x)=\|x\|^{-\alpha}$ and for a PPP. In this case the interference at the

origin is given by $I=\sum_{\mathrm{x} \in \Phi} \ell(\mathrm{x})$. We can then divide the transmitting set into two subsets, denoted as the near set and the far set. The near 


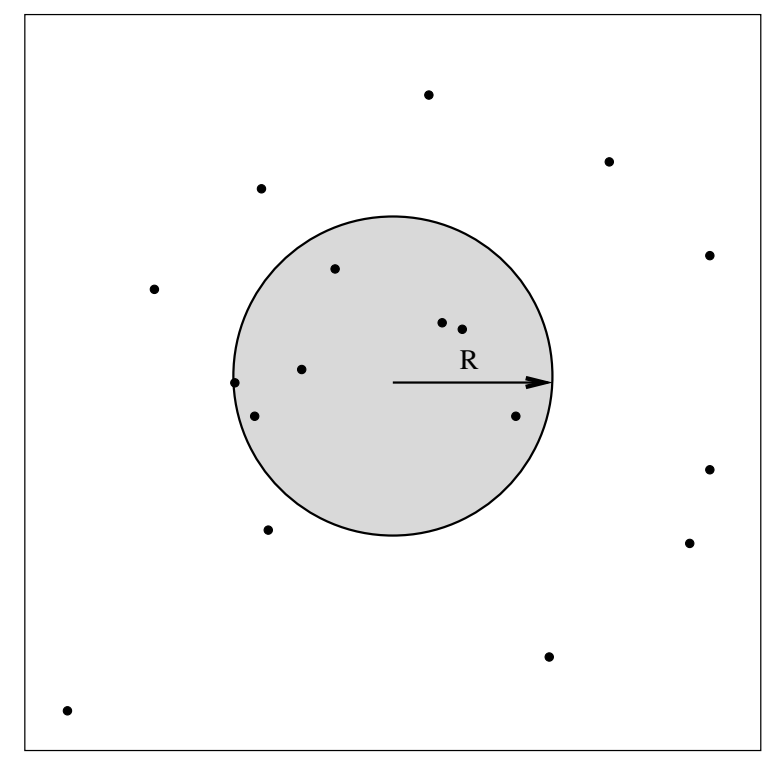

Fig. 5.1 The nodes inside the disk of radius $R=y^{-1 / \alpha}$ constitute the near set, i.e., the set of transmitters that individually contribute at least $y$ to the interference $I$ in the non-fading case.

set consists of all the nodes that individually contribute at least $y$ to $I$, and the far set is the complement of the near set. See Figure 5.1. Without fading, the near set consists of the nodes in $b\left(o, y^{-1 / \alpha}\right)$. We can lower bound $\mathbb{P}(I>y)$ by neglecting the contribution of the far set and this will be a tight bound if $\alpha-2$ is not too small since most of the contribution to the interference is from the near set. A lower bound for $\mathbb{P}(I<y)$ follows by first observing that the event $I<y$ requires the near set to be empty and secondly that the contribution of the far set can be replaced by its average using the Markov inequality (the average interference caused by the far set is finite for $y>0$ ). When there is fading there is an effective reordering of the points, as discussed in detail in [20] but the near and far sets can still be defined in a similar fashion.

Theorem 5.3. When the transmitters are distributed as a stationary point process $\Phi$, the $\operatorname{CCDF} \bar{F}_{I}(y)$ of the interference at location $z$, 
conditioned on a transmitter present at the origin but not included in the interference, is lower bounded by $\bar{F}_{I}^{l}(y)$ and upper bounded by $\bar{F}_{I}^{u}(y)$, where

$$
\begin{gathered}
\bar{F}_{I}^{l}(y)=1-\tilde{\mathcal{G}}\left[F_{h}\left(\frac{y}{\ell(.-z)}\right)\right], \\
\bar{F}_{I}^{u}(y)=1-(1-\varphi(y)) \tilde{\mathcal{G}}\left[F_{h}\left(\frac{y}{\ell(.-z)}\right)\right],
\end{gathered}
$$

where $F_{h}(x)$ denotes the CDF of the fading coefficient $h$, and

$$
\varphi(y)=\frac{1}{y \lambda} \int_{\mathbb{R}^{2}} \ell(x-z) \rho^{(2)}(x) \int_{0}^{y / \ell(x-z)} \nu \mathrm{d} F_{h}(\nu) \mathrm{d} x
$$

If $\mathbb{E}^{! o}\left[I_{\Phi}^{p}\right]<\infty$, we can also use a loose $\varphi(y)=\mathbb{E}^{! o}\left[I_{\Phi}^{p}\right] y^{-p}, \quad p \geq 1$.

Proof. The basic idea is to partition the transmitter set $\Phi$ into two subsets $\Phi_{y}$ and $\Phi_{y}^{c}$ where,

$$
\begin{array}{ll}
\Phi_{y}=\left\{\mathrm{x} \in \Phi, h_{\mathrm{x}} \ell(\mathrm{x}-z)>y\right\} & \text { (near set) } \\
\Phi_{y}^{c}=\left\{\mathrm{x} \in \Phi, h_{\mathrm{x}} \ell(\mathrm{x}-z) \leq y\right\} & \text { (far set). }
\end{array}
$$

$\Phi_{y}$ consists of those transmitters whose contribution to the interference exceeds $y$. We have $I_{\Phi}(z)=I_{\Phi_{y}}(z)+I_{\Phi_{y}^{c}}(z)$, where $I_{\Phi_{y}}(z)$ corresponds to the interference due to the transmitter set $\Phi_{y}$ and $I_{\Phi_{y}^{c}}(z)$ corresponds to the interference due to the transmitter set $\Phi_{y}^{c}$. Hence we have

$$
\begin{aligned}
\bar{F}_{I}(y) & =\mathbb{P}\left(I_{\Phi_{y}}(z)+I_{\Phi_{y}^{c}}(z) \geq y\right) \\
& \geq \mathbb{P}\left(I_{\Phi_{y}}(z) \geq y\right) \\
& =1-\mathbb{P}\left(I_{\Phi_{y}}(z)<y\right) \\
& =1-\mathbb{P}\left(\Phi_{y}=\emptyset\right) .
\end{aligned}
$$


We can find the probability $\mathbb{P}\left(\Phi_{y}=\emptyset\right)$ that $\Phi_{y}$ is empty using the conditional Laplace functional as follows:

$$
\begin{aligned}
\mathbb{P}\left(\Phi_{y}=\emptyset\right) & =\mathbb{E}^{! o} \prod_{x \in \Phi} \mathbf{1}_{h_{x} \ell(x-z) \leq y} \\
& \stackrel{(a)}{=} \mathbb{E}^{! o} \prod_{x \in \Phi} \mathbb{E}_{h}\left(\mathbf{1}_{h_{x} \ell(x-z) \leq y}\right) \\
& =\mathbb{E}^{! o} \prod_{x \in \Phi} F_{h}\left(\frac{y}{\ell(x-z)}\right) \\
& =\tilde{\mathcal{G}}\left[F_{h}\left(\frac{y}{\ell(\cdot-z)}\right)\right],
\end{aligned}
$$

where $(a)$ follows from the independence of $h_{\mathrm{x}}$. To obtain the upper bound,

$$
\begin{aligned}
\bar{F}_{I}(y)= & \mathbb{P}\left(I_{\Phi}>y \mid I_{\Phi_{y}}>y\right) \bar{F}_{I}^{l}(y)+\mathbb{P}\left(I_{\Phi}>y \mid I_{\Phi_{y}} \leq y\right)\left(1-\bar{F}_{I}^{l}(y)\right) \\
\stackrel{(a)}{=} & 1-\tilde{\mathcal{G}}\left[F_{h}\left(\frac{y}{\ell(\cdot-z)}\right)\right] \\
& +\mathbb{P}\left(I_{\Phi}>y \mid I_{\Phi_{y}} \leq y\right) \tilde{\mathcal{G}}\left[F_{h}\left(\frac{y}{\ell(\cdot-z)}\right)\right] \\
= & 1-\left(1-\mathbb{P}\left(I_{\Phi}>y \mid I_{\Phi_{y}} \leq y\right)\right) \tilde{\mathcal{G}}\left[F_{h}\left(\frac{y}{\ell(\cdot-z)}\right)\right]
\end{aligned}
$$

where $(a)$ follows from the lower bound we have established. To bound $\mathbb{P}\left(I_{\Phi}>y \mid I_{\Phi_{y}} \leq y\right)$ we use the Markov inequality. We have

$$
\begin{aligned}
\mathbb{P}\left(I_{\Phi}>y \mid I_{\Phi_{y}} \leq y\right) & =\mathbb{P}\left(I_{\Phi}>y \mid \Phi_{y}=\emptyset\right) \\
& \stackrel{(a)}{\leq} \frac{\mathbb{E}^{! o}\left(I_{\Phi} \mid \Phi_{y}=\emptyset\right)}{y} \\
& =\frac{1}{y} \mathbb{E}^{! o} \sum_{x \in \Phi} h_{x} \ell(x-z) \mathbf{1}_{h_{x} \ell(x-z) \leq y} \\
& =\frac{1}{y} \mathbb{E}^{! o} \sum_{x \in \Phi} \ell(x-z) \int_{0}^{y / \ell(x-z)} \nu \mathrm{d} F_{h}(\nu) \\
& \stackrel{(b)}{=} \frac{1}{y \lambda} \int_{\mathbb{R}^{2}} \ell(x-z) \int_{0}^{y / \ell(x-z)} \nu \mathrm{d} F_{h}(\nu) \rho^{(2)}(x) \mathrm{d} x
\end{aligned}
$$


where $(a)$ follows from the Markov inequality, and (b) follows from a procedure similar to the calculation of the mean interference in the previous section.

Since isotropy is not used in the proof, Theorem 5.3 holds for stationary point processes, not just motion-invariant ones.

When $\Phi$ is a PPP, we have $\tilde{\mathcal{G}}[v]=\exp \left(-\lambda \int 1-v(x) \mathrm{d} x\right)$ (A.3). For Rayleigh fading and $\ell(x)=\|x\|^{-\alpha}$, the lower bound is

$$
\bar{F}_{I}^{l}(y)=1-\exp \left(-\pi \lambda y^{-\delta} \Gamma(1+\delta)\right)
$$

and the upper bound is

$$
\bar{F}_{I}^{u}(y)=1-\left(1-\frac{2 \pi \lambda \Gamma(1+\delta)}{\alpha-2} y^{-\delta}\right) \exp \left(-\pi \lambda y^{-\delta} \Gamma(1+\delta)\right) .
$$

From Figure 5.2, we observe that the lower bound is closer to the actual CCDF than the upper bound. In the above derivation, the upper bound may be loose because a simple Markov inequality is used to bound $\mathbb{P}\left(I_{\Phi}>y \mid I_{\Phi_{y}} \leq y\right)$, and a better bound may be obtained by using the Chernoff bound. The upper bound diverges as $\alpha \downarrow 2$ since the average interference contribution from the far set diverges. From the upper and lower bounds we can (once again) infer that the interference in a PPP network is heavy-tailed with parameter $\delta$ for the singular path loss model.

In the next lemma we prove that the upper and lower bounds are asymptotically tight when $\ell(x)=\|x\|^{-\alpha}$. We will use $g_{1}(x) \sim g_{2}(x)$ to denote $\lim _{x \rightarrow \infty} g_{1}(x) / g_{2}(x)=1$.

Lemma 5.4. When $\ell(x)=\|x\|^{-\alpha}, \alpha>2, z \in \mathbb{R}^{2}$ and $\rho^{(2)}(z)$ continuous in a small neighborhood of $z$,

$$
\varphi(y) \begin{cases}\sim \frac{\pi \rho^{(2)}(z) \mathbb{E}\left[h^{\delta}\right]}{\lambda\left(\delta^{-1}-1\right)} y^{-\delta} & \rho^{(2)}(z) \neq 0 \\ =o\left(y^{-\delta}\right) & \rho^{(2)}(z)=0 .\end{cases}
$$




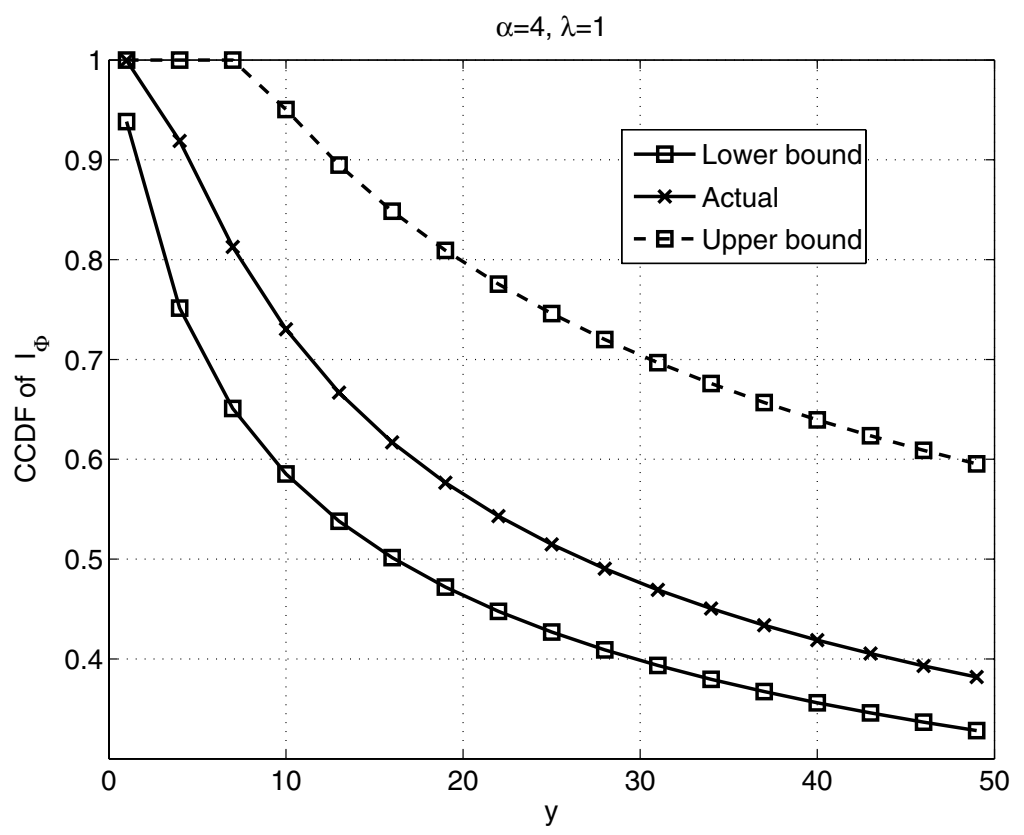

Fig. 5.2 The CCDF of the interference is plotted when $\Phi$ is a PPP and $\ell(x)=\|x\|^{-4}$. In this case the interference is a Lévy-stable distribution (3.20).

Proof. When $\ell(x)=\|x\|^{-\alpha}, \alpha>2$, we have

$$
\begin{aligned}
\varphi(y) & =\frac{1}{y \lambda} \int_{\mathbb{R}^{2}}\|x-z\|^{-\alpha} \rho^{(2)}(x) \int_{0}^{\|x-z\|^{\alpha}} \nu \mathrm{d} F_{h}(\nu) \mathrm{d} x \\
& \stackrel{(a)}{=} \frac{1}{y \lambda} \int_{0}^{\infty} \nu \int_{\mathbb{R}^{2}}\|x\|^{-\alpha} \mathbf{1}_{\|x\|^{\alpha}>\nu y^{-1}} \rho^{(2)}(x+z) \mathrm{d} x \mathrm{~d} F_{h}(\nu) \\
& \stackrel{(b)}{=} \frac{y^{-\delta}}{\lambda} \int_{0}^{\infty} \nu \int_{\mathbb{R}^{2}}\|x\|^{-\alpha} \mathbf{1}_{\|x\|^{\alpha}>\nu} \rho^{(2)}\left(\frac{x}{y^{1 / \alpha}}+z\right) \mathrm{d} x \mathrm{~d} F_{h}(\nu),
\end{aligned}
$$

where $(a)$ follows from the substitution $x \rightarrow x+z$ and interchanging the integrals. (b) follows by the substitution $y^{1 / \alpha} x \rightarrow x$. So by the dominated convergence theorem, we have for $\rho^{(2)}(z) \neq 0$

$$
\begin{aligned}
\lim _{y \rightarrow 0} \frac{\varphi(y)}{y^{-\delta}} & =\frac{\rho^{(2)}(z)}{\lambda} \int_{0}^{\infty} \nu \int_{\mathbb{R}^{2}}\|x\|^{-\alpha} \mathbf{1}_{\|x\|^{\alpha}>\nu} \mathrm{d} x \mathrm{~d} F_{h}(\nu) \\
& =\frac{2 \pi \rho^{(2)}(z) \mathbb{E}\left[h^{2 / \alpha}\right]}{\lambda(\alpha-2)} .
\end{aligned}
$$




\subsection{Asymptotic Behavior of the Interference Distribution}

In the previous section, bounds on the CCDF were provided and they depend on the conditional PGFL. But the PGFL (let alone the conditional PGFL) is known only for a few point processes. In this section we take an alternate approach and evaluate the tail of the CDF. We show that the CCDF of the interference depends critically on the path loss model $\ell(x)$.

\subsubsection{Singular Path Loss Function}

For a real-valued function $f(x)$, the behavior of the function for large $x$ can be evaluated by examining its Laplace transform at $s=0$. The generalization of this idea is expressed by the following Tauberian theorem.

Theorem 5.5 (Tauberian theorem $[5,10]$ ). For $0 \leq \beta \leq 1$, $\eta \in R_{0}$, the following are equivalent:

$$
\begin{aligned}
& 1-\int_{-\infty}^{\infty} e^{-s x} f(x) \mathrm{d} x \sim s^{\beta} \eta\left(\frac{1}{s}\right), \quad s \downarrow 0 \\
& 1-\int_{0}^{x} f(y) \mathrm{d} y \sim \frac{\eta(x)}{x^{\beta} \Gamma(1-\beta)}, \quad x \rightarrow \infty .
\end{aligned}
$$

$R_{0}$ represents the set of functions which satisfy the property

$$
\frac{\eta(\lambda x)}{\eta(x)} \rightarrow 1, \quad x \rightarrow \infty
$$

In this section we will derive the tail behavior of the interference using the conditional Laplace transform of the interference. We first prove that the interference is heavy-tailed when the path loss model is singular. The basic idea of the proof is as follows: From the previous chapter, we know that the conditional Laplace transform of the interference is given by $\tilde{\mathcal{G}}\left[\mathcal{L}_{h}(s \ell(\cdot-z))\right]$. We have

$$
\tilde{\mathcal{G}}\left[\mathcal{L}_{h}(s \ell(\cdot z))\right]=\tilde{\mathcal{G}}[1-v(s, x-z)],
$$


where $v(s, x)=\left(1-\mathcal{L}_{h}(s \ell(x))\right)$. We observe that $v(s, x) \rightarrow 0$ as $s \rightarrow 0$. Since $v(s, x)$ is small, we show that the following approximation holds when $s$ is small.

$$
\begin{aligned}
\tilde{\mathcal{G}}[1-v(s, x)] & \approx 1-\mathbb{E}^{! o} \sum_{\mathrm{x} \in \Phi} v(s, \mathrm{x}-z) \\
& =1-\int_{\mathbb{R}^{2}} v(s, x-z) \rho^{(2)}(x) \mathrm{d} x .
\end{aligned}
$$

It is then shown that $\int_{\mathbb{R}^{2}} v(s, x-z) \rho^{(2)}(x) \mathrm{d} x=\Theta\left(s^{2 / \alpha}\right)$ when $\ell(x)=$ $\|x\|^{-\alpha}$ and the Tauberian theorem is used to prove that the tail of the interference is heavy.

Theorem 5.6. Let $z \in \mathbb{R}^{2}$ such that $\rho^{(2)}(z) \neq 0$ and $\rho^{(2)}(z)$ continuous in a small neighborhood of $z$. If $\ell(x)=\|x\|^{-\alpha}, \alpha>2$, and factorial moments of $\Phi$ exist then $I_{\Phi}(z)$ is heavy-tailed with parameter $\delta$. More precisely,

$$
\mathbb{P}\left(I_{\Phi}(z) \geq y\right) \sim \frac{\pi \rho^{(2)}(z)}{\lambda} \mathbb{E}\left[h^{\delta}\right] y^{-\delta}, \quad y \rightarrow \infty
$$

Proof. The idea is to use the scaling of the Laplace transform of $I_{\Phi}(z)$ at $s=0$ and derive the properties of the tail properties of $I_{\Phi}(z)$ using the Tauberian Theorem 5.5.

Let $\ell_{r}(x)=\ell(x) \mathbf{1}_{b(o, r)}(x)$ be a truncated version of $\ell(x)$. Observe that $\ell_{r}(x) \rightarrow \ell(x)$ uniformly as $r \rightarrow \infty$. We now prove that $\mathbb{E}_{h}\left(e^{-s h \ell_{r}(x-z)}\right)$ belongs to the class of functions over which the conditional PGFL is continuous. First, we observe that $1-\mathbb{E}_{h}\left(e^{-s h \ell_{r}(x-z)}\right)$ has a bounded support. Next we prove moment convergence:

$$
\int_{\mathbb{R}^{2}}\left|\mathbb{E}_{h}\left(e^{-s h \ell_{r}(x-z)}\right)-\mathbb{E}_{h}\left(e^{-\operatorname{sh} \ell(x-z)}\right)\right| \rho^{(2)}(x) \mathrm{d} x \rightarrow 0 \quad \text { as } r \rightarrow \infty .
$$

The left-hand side of the above equation is

$$
\begin{aligned}
& \int_{\mathbb{R}^{2}}\left|\int_{0}^{\infty}\left[\exp \left(-s \ell_{r}(x-z) t\right)-\exp (-s \ell(x-z) t)\right] f(t) \mathrm{d} t\right| \rho^{(2)}(x) \mathrm{d} x \\
& \quad=\int_{\|x-z\|>r} \int_{0}^{\infty}[1-\exp (-s \ell(x-z) t)] f(t) \mathrm{d} t \rho^{(2)}(x) \mathrm{d} x
\end{aligned}
$$




$$
\begin{aligned}
& \stackrel{(a)}{\leq} s \int_{\|x-z\|>r}\|x-z\|^{-\alpha} \rho^{(2)}(x) \mathrm{d} x \int_{0}^{\infty} t f(t) \mathrm{d} t \\
& \stackrel{(b)}{=} \frac{\mathbb{E}[h] s}{r^{\alpha-2}} \int_{\|x\|>1}\|x\|^{-\alpha} \rho^{(2)}(r x+z) \mathrm{d} x \\
& \stackrel{(c)}{=} \rightarrow 0, \quad \text { when } \quad r \rightarrow \infty,
\end{aligned}
$$

where $(a)$ follows from the inequality $1-\exp (-x) \leq x,(b)$ follows from the substitution $r^{-1}(x-z) \rightarrow x$, and $(c)$ follows since $\rho^{(2)}(r x+z) \rightarrow$ $\lambda^{2}$ and $\int_{\|x\|>1}\|x\|^{-\alpha} \mathrm{d} x<\infty$.

From (5.9) and from [8, Problem 9.4.5] it follows that

$$
\mathcal{L}_{I_{\Phi}(z)}(s)=\lim _{r \rightarrow \infty} \mathbb{E}^{! o}\left[\prod_{x \in \Phi} \mathbb{E}_{h}\left(e^{-s h \ell_{r}(x-z)}\right)\right] .
$$

Hence we can work with $\ell_{r}(x)$ and take the limit $r \rightarrow \infty$ at the end. Define

$$
k_{r}(s, x)=\mathbb{E}_{h}\left(e^{-s h \ell_{r}(x-z)}\right) .
$$

Because $\ell_{r}(x)=\ell(x) \mathbf{1}_{b(o, r)}(x)$ is defined on a compact subset, we have that $k_{r}(s, x) \neq 1$ only on a compact subset $B_{r}$ of $\mathbb{R}^{2}$. Since $\Phi \cap b(o, r)$ is a simple and finite point process, $\Phi\left(B_{r}\right)<\infty$ a.s. So we have from [52, p. 458],

$$
\begin{aligned}
0 & \leq \prod_{\mathrm{x} \in \Phi}\left[1-\left(1-k_{r}(s, \mathrm{x})\right)\right]-\left[1-\sum_{\mathrm{x} \in \Phi}\left(1-k_{r}(s, \mathrm{x})\right)\right] \\
& \leq \sum_{\mathrm{x}_{1}, x_{2} \in \Phi}^{\neq}\left(1-k_{r}\left(s, \mathrm{x}_{1}\right)\right)\left(1-k_{r}\left(s, \mathrm{x}_{2}\right)\right) .
\end{aligned}
$$

First taking expectation and then taking the limit, we have from (5.11), $\lim _{r \rightarrow \infty} \mathbb{E}^{! o}\left[\prod_{\mathrm{x} \in \Phi}\left[1-\left(1-k_{r}(s, \mathrm{x})\right)\right]\right]=\mathcal{L}_{I_{\Phi}(z)}(s)$. Also,

$$
\begin{aligned}
\eta(s) & =\lim _{r \rightarrow \infty} \mathbb{E}^{! o}\left[\sum_{x \in \Phi}\left(1-k_{r}(s, \mathrm{x})\right)\right] \\
& \stackrel{(a)}{=} \lim _{r \rightarrow \infty} \lambda^{-1} \int_{\mathbb{R}^{2}}\left(1-k_{r}(s, x)\right) \rho^{(2)}(x) \mathrm{d} x \\
& =\lambda^{-1} \int_{\mathbb{R}^{2}}(1-k(s, x)) \rho^{(2)}(x) \mathrm{d} x,
\end{aligned}
$$


where $(a)$ follows from the definition of $\rho^{(2)}(x)$ and $k(s, x)=$ $\lim _{r \rightarrow \infty} k_{r}(s, x)$. Similarly,

$$
\begin{aligned}
\beta(s) & =\lim _{r \rightarrow \infty} \mathbb{E}^{! o}\left[\sum_{\mathrm{x}_{1}, \mathrm{x}_{2} \in \Phi}^{\neq}\left(1-k_{r}\left(s, \mathrm{x}_{1}\right)\right)\left(1-k_{r}\left(s, \mathrm{x}_{2}\right)\right)\right] \\
& \stackrel{(a)}{=} \lambda^{-1} \int_{\mathbb{R}^{2}} \int_{\mathbb{R}^{2}}\left(1-k\left(s, x_{1}\right)\right)\left(1-k\left(s, x_{2}\right)\right) \rho^{(3)}\left(x_{1}, x_{2}\right) \mathrm{d} x_{1} \mathrm{~d} x_{2},
\end{aligned}
$$

where $(a)$ follows from the definition of $\rho^{(3)}(x)$. We now prove that $\lim _{s \rightarrow 0} \eta(s) s^{-\delta}>0$ and $\lim _{s \rightarrow 0} \beta(s) s^{-\delta}=0$. From

$$
\begin{aligned}
\eta(s) & =\lambda^{-1} \int_{\mathbb{R}^{2}}\left[1-\mathbb{E}_{h}\left(e^{-\operatorname{sh} \ell(x-z)}\right)\right] \rho^{(2)}(x) \mathrm{d} x \\
& =\lambda^{-1} \int_{0}^{\infty} \int_{\mathbb{R}^{2}}\left[1-\exp \left(-s\|x\|^{-\alpha} t\right)\right] \rho^{(2)}(x+z) \mathrm{d} x f(t) \mathrm{d} t \\
& =\lambda^{-1} s^{\delta} \int_{0}^{\infty} \int_{\mathbb{R}^{2}}\left[1-\exp \left(-\|x\|^{-\alpha} t\right)\right] \rho^{(2)}\left(x s^{\delta}+z\right) \mathrm{d} x f(t) \mathrm{d} t .
\end{aligned}
$$

we obtain

$$
\begin{aligned}
\lim _{s \rightarrow 0} \frac{\eta(s)}{s^{\delta}}= & \lambda^{-1} \lim _{s \rightarrow 0} \int_{0}^{\infty} \int_{R^{2}}\left[1-\exp \left(-\|x\|^{-\alpha} t\right)\right] \\
& \rho^{(2)}\left(x s^{\delta}+z\right) \mathrm{d} x f(t) \mathrm{d} t \\
\stackrel{(a)}{=} & \lambda^{-1} \rho^{(2)}(z) \int_{0}^{\infty} \int_{R^{2}}\left[1-\exp \left(-\|x\|^{-\alpha} t\right)\right] \mathrm{d} x f(t) \mathrm{d} t \\
= & \lambda^{-1} \rho^{(2)}(z) \mathbb{E}\left[h^{\delta}\right] \pi \Gamma(1-\delta) .
\end{aligned}
$$

Here $(a)$ follows from the dominated convergence theorem. We now prove that $\beta(s)$ divided by $s^{\delta}$ tends to zero. Consider

$$
\begin{aligned}
\beta(s)= & \lambda^{-1} \int_{\mathbb{R}^{2}} \int_{\mathbb{R}^{2}}\left(1-k\left(s, x_{1}\right)\right)\left(1-k\left(s, x_{1}\right)\right) \rho^{(3)}\left(x_{1}, x_{2}\right) \mathrm{d} x_{1} \mathrm{~d} x_{2} \\
= & \lambda^{-1} s^{4 / \alpha} \int_{\mathbb{R}^{2}} \int_{\mathbb{R}^{2}}\left(\int_{0}^{\infty} 1-\exp \left(-\left\|x_{1}\right\|^{-\alpha} t\right) f(t) \mathrm{d} t\right) . \\
& \left(\int_{0}^{\infty} 1-\exp \left(-\left\|x_{2}\right\|^{-\alpha} t\right) f(t) \mathrm{d} t\right) . \\
& \rho^{(3)}\left(s^{1 / \alpha} x_{1}+z, s^{1 / \alpha} x_{2}+z\right) \mathrm{d} x_{1} \mathrm{~d} x_{2} .
\end{aligned}
$$


The integral is finite because $\rho^{(3)}(x, y)<\log (\|x\|) \log (\|y\|)$ for large $|x|$ and $|y|$. Hence

$$
\lim _{s \rightarrow 0} \frac{\beta(s)}{s^{\delta}}=0
$$

From (5.12), we have

$$
0 \leq \mathcal{L}_{I_{\Phi}(z)}(s)-(1-\eta(s)) \leq \beta(s) .
$$

Dividing both sides by $s^{\delta}$, taking the limit $s \rightarrow 0$ and using (5.13) and (5.14),

$$
\lim _{s \rightarrow 0} \frac{1-\mathcal{L}_{I_{\Phi}(z)}(s)}{s^{\delta}}=\frac{\pi \rho^{(2)}(z)}{\lambda} \mathbb{E}\left[h^{\delta}\right] \Gamma(1-\delta) .
$$

So we have

$$
1-\mathcal{L}_{I_{\Phi}(z)}(s) \sim \frac{\pi \rho^{(2)}(z)}{\lambda} \mathbb{E}\left[h^{\delta}\right] \Gamma(1-\delta) s^{\delta} .
$$

So using the Tauberian Theorem 5.5,

$$
\mathbb{P}\left(I_{\Phi}(z) \geq y\right) \sim \frac{\pi \rho^{(2)}(z)}{\lambda} \mathbb{E}\left[h^{\delta}\right] y^{-\delta} .
$$

From the above theorem we observe that interference at $z$ is always heavy-tailed with parameter $\delta$ when the path loss model is singular and $\rho^{(2)}(z) \neq 0$. This is because the receiver is not a part of the process and a transmitter can be arbitrarily close to it, which causes the interference to be very large with finite probability. This is consistent with (3.25).

\subsubsection{Non-singular Path Loss Function}

We now investigate the interference distribution when the path loss is non-singular or bounded. In this case, the existence of an interferer close to a receiver will not alter the magnitude of the interference drastically. Instead the fading becomes an important factor, and the distribution tail depends mainly on the tail of the fading. We will use the following theorem that connects the convergence region of the Laplace transform of a random variable and the decay of its CCDF. 
Theorem 5.7 (Nakagawa). Let $X$ be a non-negative random variable, and $F(x)=\mathbb{P}(X \leq x)$ be the probability distribution function of $X$. Let

$$
\mathcal{L}_{X}(s)=\int_{0}^{\infty} e^{-s x} \mathrm{~d} F(x), s=\sigma+j \tau \in \mathbb{C}
$$

be the Laplace-Stieltjes transform of $F(x)$ and $\sigma_{0}$ be the abscissa of convergence of $\mathcal{L}_{X}(s)$. We assume $-\infty<\sigma_{0}<0$. If $s=\sigma_{0}$ is a pole of $\mathcal{L}_{X}(s)$, then we have

$$
\lim _{x \rightarrow \infty} \frac{1}{x} \log \mathbb{P}(X>x)=\sigma_{0} .
$$

The above theorem is useful in dealing with fading distributions whose tail decays exponentially. Since the conditional Laplace transform of the interference is known, it can be used in conjunction with the above theorem to prove the exponential decay of the interference tail when the fading is exponential. When the fading distribution is heavy-tailed, we use the upper and lower bounds on the CCDF provided in Theorem 5.3 to prove that the interference is also heavy-tailed.

Theorem 5.8. Let $\ell(x)=1 /\left(1+\|x\|^{\alpha}\right)$. Then

(1) If the fading has at most an exponential tail, i.e., $\bar{F}_{h}(x)<$ $\exp (-a x)$ for large $x$, then the interference tail is also exponential. Formally: if $\exists a>0$ s.t. $\bar{F}_{h}(x)=\Theta\left(e^{-a x}\right), x \rightarrow \infty$, this implies $\bar{F}_{I}(x)=\Theta\left(e^{-a x}\right)$.

(2) If the fading is heavy-tailed, the interference is also heavytailed:

$$
\bar{F}_{h}(x) \sim x^{-\alpha} \Longrightarrow \bar{F}_{I}(x) \sim x^{-\alpha}
$$

Proof. Case (1): Exponential fading. We will first show that the conditional Laplace transform of the interference converges for $s<\sigma, \sigma<0$ and diverges for $s>\sigma$. We have

$$
\mathcal{L}_{I_{\Phi}(z)}(s)=\mathbb{E}^{! o} \prod_{\mathrm{x} \in \Phi} k(s, \mathrm{x})
$$


where $k(s, x)=\mathcal{L}_{h}(s \ell(x-z))$. From the above equation we observe that $\mathcal{L}_{I_{\Phi}(z)}(s)$ is finite if and only if

$$
\eta(s)=\mathbb{E}^{! o} \sum_{\mathrm{x} \in \Phi}|\log k(s, \mathrm{x})|<\infty .
$$

We now show that the abscissa of convergence $\sigma$ of $\mathcal{L}_{I_{\Phi}(z)}(s)$ is strictly less than zero, i.e., $\eta(s)<\infty$ for some $s<0$.

Let $\beta(s, x, h)=\exp \left(\frac{-s h}{\left(1+\|x-z\|^{\alpha}\right)}\right)$. We have

$$
\begin{aligned}
\eta(s) & =\mathbb{E}^{! o} \sum_{\mathrm{x} \in \Phi}|\log k(s, \mathrm{x})| \\
& =\int_{\mathbb{R}^{2}}\left|\log \left(\mathbb{E}_{h}[\beta(s, x, h)]\right)\right| \rho^{(2)}(x) \mathrm{d} x .
\end{aligned}
$$

When $s>0$, it is trivial to see that $\eta(s)<\infty$. The rest of the proof can be better understood by considering $s<0$. Since $\bar{F}_{h}(x) \sim \exp (-a x)$, $x \rightarrow \infty$, it can be assumed without any loss of generality that the fading $\mathrm{PDF}$ is $f(x)=a \exp (-a x), x>R$, for some large $R$. We have

$$
\begin{aligned}
k(s, x)= & \int_{0}^{R} \beta(s, x, t) \mathrm{d} F(t) \\
& +a \int_{R}^{\infty} \exp \left(-t\left[a+\frac{s}{\left(1+\|x-z\|^{\alpha}\right)}\right]\right) \mathrm{d} t .
\end{aligned}
$$

The first term is always finite. Considering the second term, we observe that the term in the exponent will be positive for all $x$ when $s>-a$. Hence the integral converges when $s>-a$ and $k(s, x)$ is well-defined (especially in the neighborhood of $z$ ). ${ }^{1}$

Also observe that $k(s, x)>1$ for $s \in(-a, 0)$. Let $b \in(-a, 0)$. We now prove that $\eta(b)<\infty$. Since $k(b, x)>1$, we have $\log (k(b, x)) \leq k(b, x)-$ 1. Hence

$$
\begin{aligned}
\eta(s) & \leq \int_{\mathbb{R}^{2}}[k(b, x)-1] \rho^{(2)}(x) \mathrm{d} x \\
& =\int_{b(o, \kappa)}[k(b, x)-1] \rho^{(2)}(x) \mathrm{d} x+\int_{b(o, \kappa)^{c}}[k(b, x)-1] \rho^{(2)}(x) \mathrm{d} x
\end{aligned}
$$

\footnotetext{
${ }^{1}$ Observe the importance of 1 in the denominator of the second term. Without it $\forall s<0$, $k(s, x)$ would be undefined in an open neighborhood of $z$.
} 
for $\kappa \in(0, \infty)$. Since $b>-a, k(b, x)$ is a well-behaved function, i.e., bounded and smooth, and hence the first term in the above equation is bounded for any $\kappa<\infty$. So if we prove that the second term is finite, then $\eta(b)<\infty$. For large $\|x\|$ we have $\rho^{(2)}(x) \rightarrow \lambda^{2}$. We choose $\kappa$ such that for all $\|x\|>\kappa, \rho^{(2)}(x)$ is very close to $\lambda^{2}$. Hence $\rho^{(2)}(x)$ is essentially a constant and proving

$$
\int_{b(o, \kappa)^{c}}(k(b, x)-1) \mathrm{d} x<\infty
$$

will be sufficient. We have

$$
\begin{aligned}
\int_{b(o, \kappa)^{c}}(k(b, x)-1) \mathrm{d} x= & \underbrace{\int_{b(o, \kappa)^{c}} \int_{0}^{R}[\beta(-|b|, x, t)-1] f(t) \mathrm{d} t \mathrm{~d} x}_{\mathrm{A}} \\
& +\underbrace{\int_{b(o, \kappa)^{c}} \int_{R}^{\infty}[\beta(-|b|, x, t)-1] f(t) \mathrm{d} t \mathrm{~d} x}_{\mathrm{B}} .
\end{aligned}
$$

Considering the first term $\mathrm{A}$, we can increase $\kappa$ such that

$$
\beta(-|b|, x, t)=\exp \left(\frac{|b| t}{\left(1+\|x-z\|^{\alpha}\right)}\right) \approx 1+\frac{|b| t}{\left(1+\|x-z\|^{\alpha}\right)} .
$$

This can be done since $t<R$. Hence we have

$$
\mathrm{A}=\int_{b(o, \kappa)^{c}} \int_{0}^{R} \frac{|b| t}{\left(1+\|x-z\|^{\alpha}\right)} f(t) \mathrm{d} t \mathrm{~d} x<\infty .
$$

Considering the second integral B, substituting for the fading PDF and after some algebraic manipulation, we get

$$
\begin{aligned}
\mathrm{B}= & e^{-a R} \int_{b(o, \kappa)^{c}} \frac{a\left(1+\|x-z\|^{\alpha}\right)(\beta(-|b|, x, R)-1)}{a\left(1+\|x-z\|^{\alpha}\right)-|b|} \mathrm{d} x \\
& +e^{-a R} \int_{b(o, \kappa)^{c}} \frac{|b|}{a\left(1+\|x-z\|^{\alpha}\right)-|b|} \mathrm{d} x .
\end{aligned}
$$

Since $\kappa$ is large, using the approximation (5.18), it follows that $\mathrm{B}<\infty$. So we have shown that $\eta(b)<\infty$ for all $b \in(-a, \infty)$. We also observe that $\eta(s)=\infty$ for $s<-a$. So the abscissa is equal to $-a<0$. From [36, Theorem 3], it then follows that the tail falls exponentially with parameter $a$. 
Case (2): $\bar{F}_{h} \sim h^{-a}$ is a heavy-tailed distribution. In this case $k(s, x)=\infty$ for all $s<0$, and hence [36, Theorem 3] cannot be applied. We will use Theorem 5.3 that provides upper and lower bounds for the CCDF of the interference. We first evaluate $\tilde{\mathcal{G}}\left[F_{h}\left(\frac{y}{\ell(.-z)}\right)\right]$ for large $y$ :

$$
\begin{aligned}
\tilde{\mathcal{G}}\left[F_{h}\left(\frac{y}{\ell(.-z)}\right)\right] & =\tilde{\mathcal{G}}\left[1-\left[1-F_{h}\left(y\left(1+\|x-z\|^{\alpha}\right)\right)\right]\right] \\
& \stackrel{(a)}{\sim} \tilde{\mathcal{G}}\left(1-\left[y\left(1+\|x-z\|^{\alpha}\right)\right]^{-a}\right) \\
& \stackrel{(b)}{\sim} 1-y^{-a} \lambda^{-1} \int_{\mathbb{R}^{2}}\left[\left(1+\|x-z\|^{\alpha}\right)\right]^{-a} \rho^{(2)}(x) \mathrm{d} x,
\end{aligned}
$$

where $(a)$ follows by the continuity of $\tilde{\mathcal{G}}$ and the fact that $y$ is large. (b) follows from an argument similar to Theorem 5.6. We also have from Theorem 5.3, that $\varphi(y)=y^{-a} \mathbb{E}^{! o}\left[I_{\Phi}(z)^{a}\right]$. Here $\mathbb{E}^{! o}\left[I_{\Phi}(z)^{a}\right]<\infty$ because of the bounded nature of $\ell(x)$ and its sufficiently fast decaying tail. So from the upper bound in Theorem 5.3, we have

$$
\begin{aligned}
& \mathbb{P}\left(I_{\Phi}(z)>y\right) \\
& \quad<y^{-a}\left[\lambda^{-1} \int_{\mathbb{R}^{2}}\left[\left(1+\|x-z\|^{\alpha}\right)\right]^{-\alpha} \rho^{(2)}(x) \mathrm{d} x+\mathbb{E}^{! o}\left[I_{\Phi}(z)^{a}\right]\right],
\end{aligned}
$$

and from the lower bound

$$
\mathbb{P}\left(I_{\Phi}(z)>y\right)>y^{-a} \lambda^{-1} \int_{\mathbb{R}^{2}}\left[\left(1+\|x-z\|^{\alpha}\right)\right]^{-\alpha} \rho^{(2)}(x) \mathrm{d} x .
$$

So the tail decays like $y^{-a}$.

We now show that the distribution of interference decays exponentially fast at the origin. The basic idea is that there is some contribution from some point of the process, however, small it is.

Theorem 5.9. The CDF of the interference decays faster than any polynomial at the origin, i.e., $\forall n \in \mathbb{N}$,

$$
\mathbb{P}\left(I_{\Phi}(z)<y\right)=o\left(y^{n}\right), \quad y \rightarrow 0 .
$$


Proof. Find $a$ such that $F_{h}(x)=o\left(x^{a}\right)$. Choose $k \in \mathbb{N}$ such that $k>$ $n / a$. From Theorem 5.3, we have

$$
\begin{aligned}
\mathbb{P}\left(I_{\Phi}(z)<y\right) & <\tilde{\mathcal{G}}\left(F_{h}\left(\frac{y}{\ell(.-z)}\right)\right) \\
& =\mathbb{E}^{! o}\left[\prod_{x \in \Phi} F_{h}\left(\frac{y}{\ell(\mathrm{x}-z)}\right) \mid \Phi \text { has at least } k \text { points }\right] .
\end{aligned}
$$

Multiply both sides by $y^{-n}$ and take the limit $y \rightarrow 0$. On the right-hand side, the limit can be moved inside the expectation by the dominated convergence theorem. Since there are at least $k$ points almost surely on the plane (because $\Phi$ is stationary), we have that the limit on the right goes to zero by our choice of $k$.

\subsection{Examples and Simulation Results}

In this section we give examples of the interference for different point processes and fading distributions and give approximations for the distribution.

\subsubsection{Examples}

We concentrate on three different point processes: the PPP, the Thomas cluster process, and the shifted lattice process.

Poisson point processes. (1) Singular path loss model: $\ell(x)=$ $\|x\|^{-\alpha}$

From Section 3, the Laplace transform of the interference is $\mathcal{L}_{I_{\Phi}}(s)=$ $\exp \left(-\lambda \pi s^{\delta} \mathbb{E}\left[h^{\delta}\right] \Gamma(1-\delta)\right)$. The Laplace transform is independent of $z$, hence the distribution is independent of the location $z$. Also $\mathcal{L}_{I_{\Phi}}(s)$ is the Laplace transform of a stable random variable with parameter $\delta$ and hence heavy-tailed.

(2) Bounded path loss model: $\ell(x)=\left(1+\|x\|^{\alpha}\right)^{-1}$

We first consider the case of exponential fading, i.e., $f_{h}(x)=$ $\mu \exp (-\mu x)$. In this case,

$$
\mathcal{L}_{I_{\Phi}}(s)=\exp \left(-\lambda \pi \frac{\pi \delta}{\sin (\pi \delta)} \frac{s}{(\mu+s)^{1-\delta}}\right) .
$$




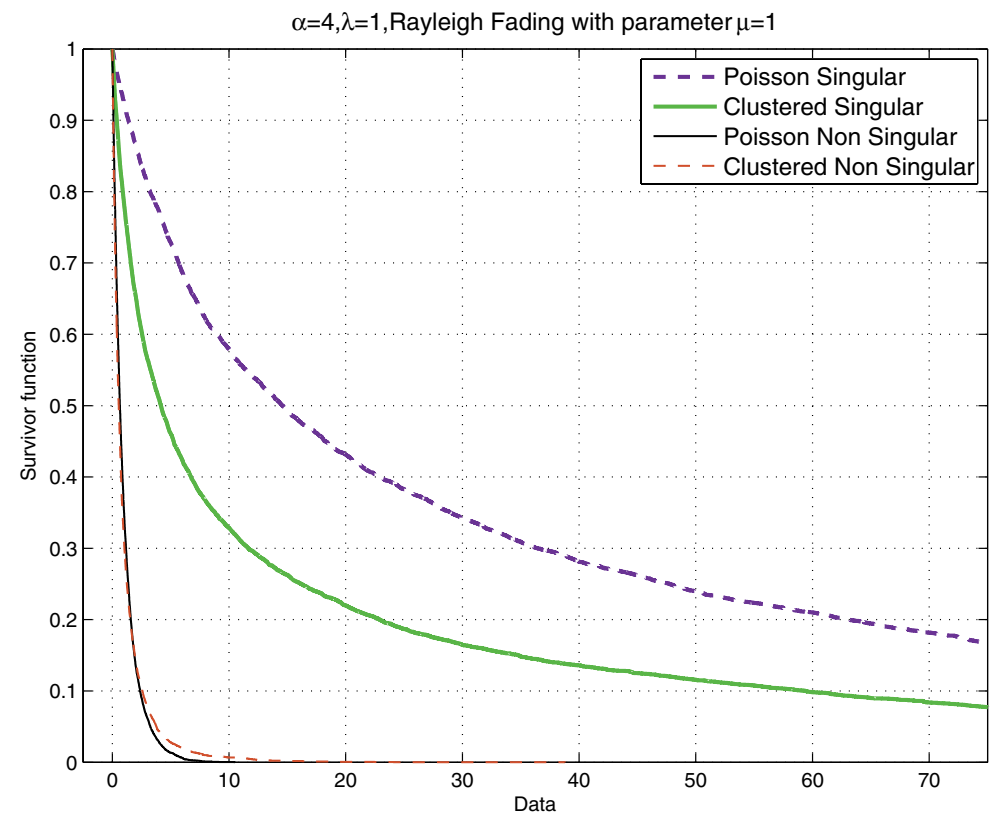

Fig. 5.3 CCDF of the interference for Rayleigh fading and path loss $\alpha=4, z=(3,0)$ (from [12] (C) 2008 IEEE).

Observe that $\mathcal{L}_{I_{\Phi}}(s)$ is well defined for $s>-\mu$. With $f_{I_{\Phi}}(x)$ being the $\mathrm{PDF}$ of the interference, we have by the final-value theorem that $\lim _{x \rightarrow \infty} e^{\mu_{1} x} f_{I_{\Phi}}(x)=\lim _{s \rightarrow 0} \mathcal{L}_{I_{\Phi}}\left(s-\mu_{1}\right)<\infty$ for all $\mu_{1}<\mu$. So the $\mathrm{PDF}$ is a combination of many decaying exponentials. In Figure 5.3, we plot the CCDF of Poisson interference with Rayleigh fading. We observe the heavy-tailed distribution for $\ell(x)=\|x\|^{-\alpha}$ and the exponential decay when $\ell(x)=\left(1+\|x\|^{\alpha}\right)^{-1}$.

Thomas cluster processes. In Section 4, we have derived the properties of interference for this distribution of nodes. We show in $[13$, Lemma 3] that the interference has a heavy-tailed distribution for $\ell(x)=\|x\|^{-\alpha}$ with parameter $\delta$.

Shifted lattice processes. The interference results for this process are verified by simulation. In Figure 5.4, we plot the CCDF for different values of $z$ and with Rayleigh fading. We observe that the tail 


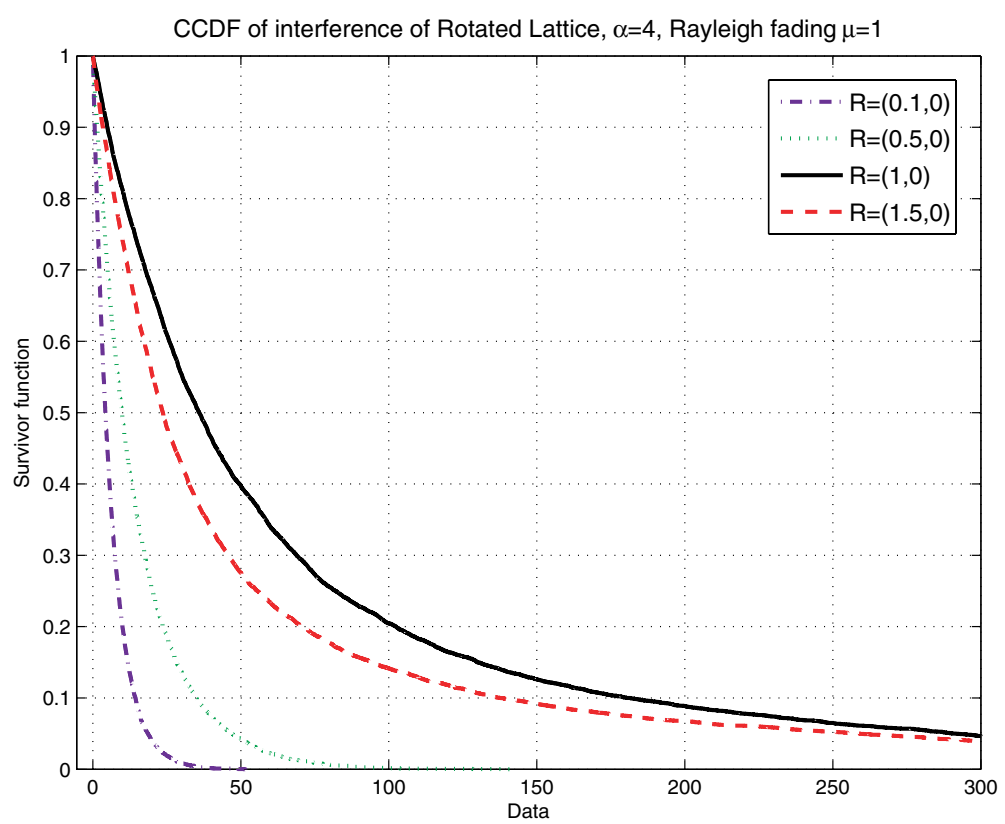

Fig. 5.4 CCDF of the interference for exponential power fading and path loss $\alpha=4$ for lattice processes (from [12] (C) 2008 IEEE).

properties depend heavily on $z$. When $\|z\|<1$, we have that $\rho^{(2)}(z)=0$ (actually the associated measure $\mathcal{K}_{2}(A)=0, \forall A \subset b(o, 1)$ ). So here the effective path loss model is bounded and hence the interference tail follows that of the fading. When $\|z\|>1$, there is a positive probability that a transmitting node can be arbitrarily close to $z$ and hence the interference follows a heavy tail distribution.

\subsubsection{Approximation of the Interference Distribution}

From the previous theorems, we have the following observations:

(1) The $\mathrm{CDF} F_{I}(y)$ of the interference decays faster than any polynomial as $y \downarrow 0$.

(2) When $\ell(x)=\left(1+\|x\|^{\alpha}\right)^{-1}, \alpha>2$, the mean interference is finite, and the CCDF tail decays like that of the fading distribution. 
(3) When $\ell(x)=\|x\|^{-\alpha}$, the mean diverges, and the CDF has a heavy tail.

Observation 1 eliminates the use of Gaussian distribution to model the interference except when the mean $\mu=\mathbb{E}\left[I_{\Phi}\right]$ is very large (but finite), so that $\exp \left(-\mu^{2} / 2 \sigma^{2}\right)$ is small. We choose three probability distributions which have the desired properties. The gamma distribution, the inverse Gaussian distribution, and the inverse gamma distribution.

(1) Gamma distribution: $f(x)=x^{k-1} \exp (-x / a) / \Gamma(k) a^{k}$. Mean: $k a$, variance: $k a^{2}$.

(2) Inverse Gaussian distribution:

$$
f(x)=\left[\frac{\nu}{2 \pi x^{3}}\right]^{1 / 2} \exp \left(-\frac{\kappa(x-\nu)^{2}}{2 \nu^{2} x}\right)
$$

Mean: $\nu$, variance: $\nu^{3} / \kappa$.

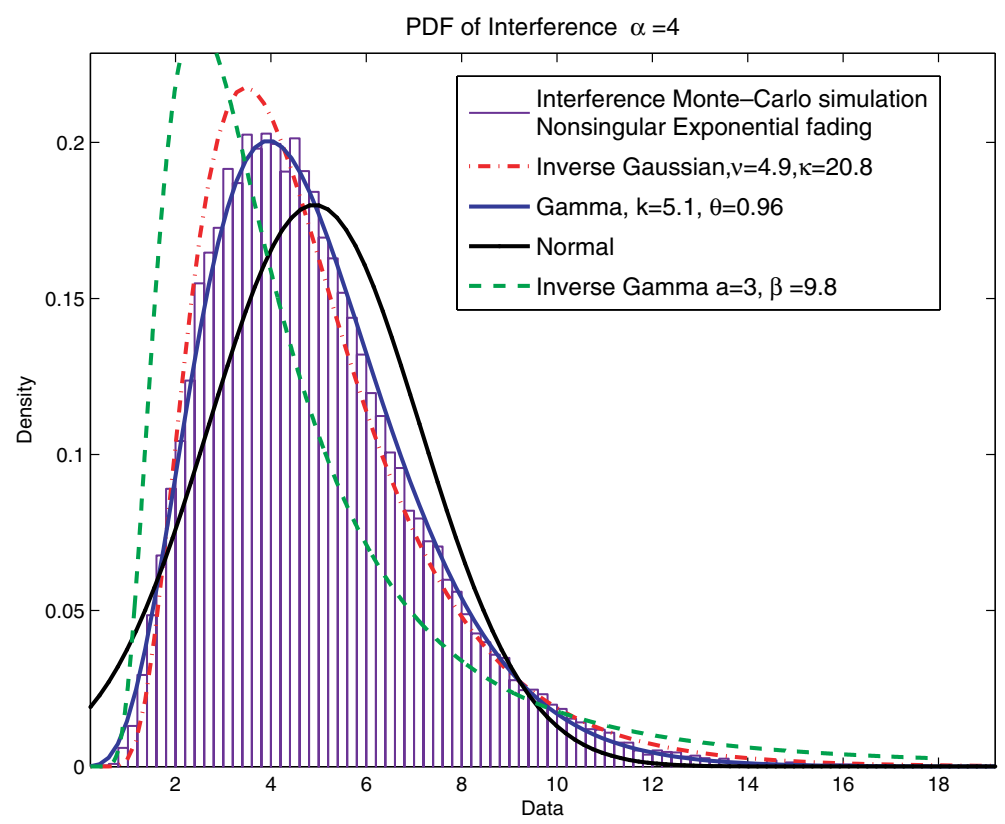

Fig. 5.5 Empirical PDF of the interference and the corresponding fits for $\ell(x)=(1+$ $\left.\|x\|^{4}\right)^{-1}$ and Rayleigh fading (mean 1) (from [12] (C) 2008 IEEE). 
(3) Inverse gamma distribution:

$$
f(x)=\beta^{a} x^{-a-1} \exp (-\beta / x) \Gamma(a)^{-1}
$$

Mean: $\beta /(a-1)$, variance: $\beta^{2} /\left((a-1)^{2}(a-2)\right)$.

In the inverse Gaussian distribution, the mean and variance can be chosen independently of each other. The gamma distribution only has a $(k-1)$-th order of decay at the origin and has an exponential tail. On the other hand, the inverse Gaussian distribution has an exponential decay at origin and a slightly super-exponential tail. In Figure 5.5, we have plotted ${ }^{2}$ the PDF of the interference using Monte-Carlo simulation when the underlying node distribution is PPP and the fading is Rayleigh with a non-singular path loss model. We observe that the normal fit performs the worst. Both the gamma and inverse Gaussian give us a good fit. The inverse gamma PDF is a bad fit since it has a fourth-order decaying tail, while the fading is exponentially decaying. In finite networks, where the number of nodes is finite and fixed and the nodes are distributed on a bounded subset of the Euclidean plane, the interference does not decay superpolynomially fast at the origin, but only goes to zero like $y^{n a}$ where $n$ is the number of transmitters and $a$ the decay of the fading CDF at the origin.

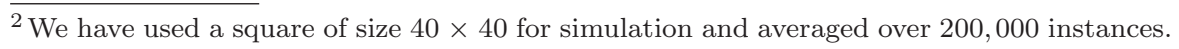




\section{6}

\section{Conclusions}

The cumulated interference power in wireless networks can be sharply characterized in certain cases. Most notably, in the case of a homogeneous Poisson network, at least the Laplace transform of the interference can be derived. For the power law path loss with singularity at the origin, the interference follows a stable distribution if the path loss exponent $\alpha$ exceeds the number of network dimensions $d$, otherwise the interference is infinite a.s. This result holds irrespective of the fading statistics and the number of network dimensions, and it implies that the mean interference does not exist. The dimensionality of the network and the path loss exponent enter the Laplace transform only through their ratio $\delta=d / \alpha$, which is exactly the characteristic exponent of the stable distribution, and the fading process only through its $\delta$-th moment. The Laplace transform, evaluated at the SIR threshold

$\theta$, is the success probability of a transmission where the desired signal power is exponentially distributed, e.g., due to Rayleigh fading. So in this case, the SIR distribution is known for all parameters $\alpha$ and $d$, whereas the $\mathrm{PDF}$ of the interference only exists when the ratio is $\delta=1 / 2$. 


\section{Conclusions}

ALOHA is the only MAC scheme that preserves the Poisson distribution, since it performs independent thinning from the process of all nodes to the process of transmitting nodes. Accordingly, ALOHA has naturally received considerable attention in the literature, see, e.g., $[2,3,51]$. In addition to its analytical advantages, ALOHA also serves as an important benchmark, since it is the only completely distributed and overhead-free MAC scheme. A more elaborate channel access mechanism may appear to yield much higher throughput, but the induced communication overhead needs to be subtracted, and the net gain relative to ALOHA can be used as an objective metric. It is also interesting to note that ALOHA achieves the $\Theta(1 / \sqrt{n})$ scaling law, so from that perspective, nothing is lost if ALOHA is employed in the analysis. Due to its independent thinning property, ALOHA is also the extreme case for the interference correlation: non-ALOHA MACs also introduce stronger correlations, so the results in correlation section (Section 3.8) are lower bounds.

As a generalization to the ubiquitous Poisson model, we have derived interference results for Poisson cluster processes in Section 4. Not surprisingly, clustering increases the interference, since the local density of interferers is higher than for the homogeneous PPP of the same overall density. On the other hand, as shown in [53], clustering can be beneficial if spread-spectrum communication is used.

The counterparts of cluster processes are hard-core processes, where a minimum separation between nodes is enforced. While such processes are a natural model for networks with CSMA-type MAC schemes, their analysis is complicated by the fact that probability generating functionals do not exist. As in many cases, there is tension between the analytical tractability and the practicality of the models. As long as motion-invariance, i.e., stationarity and isotropy, is preserved, precise statements about the interference are still possible. In Section 5 we have shown that the heavy tail of the interference is present whenever the point process model (and MAC scheme) allows two nodes to be arbitrarily close and the path loss law is singular. If either of these two conditions is not met, the tail of the interference is governed by the fading distribution. This is intuitive, since if the interference is bounded, either by a minimum distance between nodes or by the path loss law, 
the only reason why the interference can get large is fading. So great care should be exercised when choosing the network models, both for theoretical and simulation studies, to ensure that the models are not used outside the regime they are intended for.

The interference, SIR, and outage results are fundamental local physical-layer metrics, which enable the calculation of global and higher-layer metrics such as the maximum spatial throughput, optimum routing progress, and end-to-end delay [3, 21, 44]. Similarly, the derivation of asymptotic results (scaling laws) requires knowledge of the interference or SIRs, typically in the form of bounds. The point process and path loss models are equally critical for these types of analyses. Some results, in particular for so-called dense networks where the node density goes to infinite while the area remains constant, rely on the homogeneity of the path loss law, which makes nearest-neighbor communication possible irrespective of the network density [16, 17]. For a bounded path loss law, the situation changes drastically. Indeed, as shown in [9], the capacity scaling law changes to from $\Theta(1 / \sqrt{n})$ to $\Theta(1 / n)$. For a detailed account on how the singularity of the path loss model affects network performance, see [27].

The interference and outage analyses in this monograph may help devise guidelines for the design of large wireless system. Some examples of design decisions facilitated by the theory developed are:

- Improved receivers can be designed that are tailored to the interference statistics encountered. These statistics also determine when it is optimum to consider interference as noise [42].

- Transmission to the nearest neighbor in a PPP is successful with a probability that does not go to zero with increasing network density. This is the reason why multihopping over nearby neighbors is successful even in highly dense network.

- The optimum ALOHA transmit probability can be derived by maximizing the unconditional success probability $p(1-p)$ $p_{s}(p)$, which includes the probabilities that the transmitter actually transmits and the receiver listens [21]. The sensitivity to the path loss exponent shows that transmit 
probabilities need to be drastically reduced if the path loss exponent is close to the number of network dimensions. Other metrics to maximize include the spatial density of progress or spatial throughput [3].

- The density of transmitters can be maximized under an outage constraint. This is the purpose of the transmission capacity framework [51].

- SINR thresholds can be optimized if the rate of transmission is factored in by putting $R=\log _{2}(1+\theta)$ or $R=\mathbb{E}\left(\log _{2}(1+\right.$ SINR)) and maximizing the product of rate and success probability $R p_{s}(R)$. The same framework also permits the analysis of the benefits of adaptive modulation.

- Different physical-layer techniques, including spreadspectrum techniques, power control, and interference cancellation, can be analyzed [1, 48, 49].

- The correlation structure of the interference is important when designing ARQ mechanism. The positive correlation of subsequent transmission success events means that the typical assumption of an independent success when retransmitting is invalid. In a static network, the probability that the $k$-th transmission succeeds if the first $k-1$ failed goes to zero as $k \rightarrow \infty$, even with ALOHA and iid block Rayleigh fading.

- The impact of clustering can be assessed. The analysis of clustered networks yields the conditions under which it is beneficial to cluster users, or use a MAC scheme that induces clustering.

- Generally, the results for the ALOHA MAC with singleuser receivers provide lower bounds for the achievable performance. More sophisticated solutions at the lower layers can be compared with this benchmark. In some cases, when the necessary control or overhead traffic for the improved coordination is taken into account, it may turn out that the simplest schemes actually perform comparatively well. 


\section{Mathematical Preliminaries}

In this appendix, we provide a brief summary of the main mathematical techniques and concepts used: Point process theory, Palm theory, and a characterization of stable random variables.

\section{A.1 Point Process Theory}

In a wireless network, the geographical locations of the nodes is generally modelled as a point process on the plane and here we review some basic mathematical foundations of point process theory. For a detailed description and analysis of point processes, the reader is referred to [45] and $[8]$.

Let $\mathrm{N}$ be the set of all sequences of points in $\mathbb{R}^{d}$, such that any sequence $\phi \in \mathrm{N}$ :

- is finite, i.e., has only a finite number of points in any bounded subset of $\mathbb{R}^{d}$; and

- is simple, i.e., $x \neq y$ for any $x, y \in \phi$.

Informally a point process on $\mathbb{R}^{d}$ is random variable which takes values from the set of simple and finite sequences $\mathrm{N}$. The formal definition of 
point processes can be best understood by its relation to a real-valued random variable.

Numerical random variable. Let $X$ be a real-valued random variable on $(\Omega, \mathcal{A}, \mathbb{P})$, i.e., an $\mathcal{A}$-measurable function $X: \Omega \rightarrow \mathbb{R}$. The distribution of $X$ is the measure

$$
F \triangleq \mathbb{P} \circ X^{-1}
$$

on $(\mathbb{R}, \mathcal{B})$, where $\mathcal{B}$ is the Borel sigma algebra of $\mathbb{R}$, defined by

$$
F(B)=\mathbb{P} \circ X^{-1}(B)=\mathbb{P}(X \in B) \quad \forall B \in \mathcal{B} .
$$

So measurability is the requirement that $X^{-1}(B) \in \mathcal{A}$ for all $B \in \mathcal{B}$. For a numerical random variable $X: \Omega \rightarrow \mathbb{R}$, we almost always focus on the distribution function of $X$ :

$$
F(x) \triangleq F((-\infty, x])=\mathbb{P}(X \leq x) \quad \text { (right-continuous). }
$$

Point process. We shall use the notation $\phi(B), \phi \in \mathrm{N}$ and $B \subset \mathbb{R}^{d}$, to denote the number of points of $\phi$ in $B$. Let $\mathcal{N}$ denote the smallest sigma algebra so that the maps $\phi \rightarrow \phi(B)$ are measurable for all Borel subsets $B$ of $\mathbb{R}^{d} . \mathcal{N}$ is the equivalent of the Borel sigma algebra on the real line for the set of point sequences $\mathrm{N}$.

Definition A.1. A point process $\Phi$ on $\mathbb{R}^{d}$ is a measurable mapping from a probability space $(\Omega, \mathcal{A}, \mathbb{P})$ to $(\mathrm{N}, \mathcal{N})$, i.e.,

$$
\Phi: \Omega \rightarrow \mathrm{N} .
$$

So a point process is a random variable that takes values in the set of sequences $N$. Each elementary outcome $\omega \in \Omega$ determines an entire point sequence $\Phi(\omega)$. The distribution of $\Phi$ is

$$
\mathbf{P}(E)=\mathbb{P} \circ \Phi^{-1}(E)=\mathbb{P}(\Phi \in E) \quad \forall E \in \mathcal{N} .
$$

Measurability requires that $\Phi^{-1}(E) \in \mathcal{A}$. An element of $\mathcal{N}$ is an event and can be viewed as a property of the point sequence. For example in a wireless network, $Y \in \mathcal{N}$ may represent the event that there are 10 
wireless nodes in a unit ball around the origin, or it can represent the event that the minimum distance between any pair of nodes is greater than unity.

Random measure representation. Alternatively, a simple point process can be decomposed as the sum of discrete measures, i.e.,

$$
\Phi=\sum_{i=1}^{\infty} \delta_{\mathrm{x}_{i}}
$$

where $\delta_{x}$ is the Dirac measure $\delta_{x}(B)=\mathbf{1}_{B}(x)$ for $B \in \mathcal{B}$.

Void probabilities. A simple point process is fully characterized by its void probability on compact subsets of $\mathbb{R}^{d}$, i.e., $\mathbb{P}(\Phi(K)=0)$ for compact subsets $K$ of $\mathbb{R}^{d}$. So to verify the equivalence of two simple point processes, it is sufficient to check the equality of their void probabilities on all compact sets.

We now provide some examples of point processes.

\section{A.1.1 Binomial Point Process (BPP)}

A BPP is generally used to model the location of a fixed number of wireless nodes in a bounded domain. It is a simple point process obtained by placing $n$ points $\Phi=\left\{\mathrm{x}_{1}, \ldots, \mathrm{x}_{n}\right\} \subset \mathbb{R}^{d}$ independently and uniformly in a closed and bounded set $B \subset \mathbb{R}^{d}$. The probability that there are $k<n$ nodes in $A \subset B$ of a BPP is

$$
\mathbb{P}(\Phi(A)=k)=\left(\begin{array}{l}
n \\
k
\end{array}\right)\left(\frac{|A|}{|B|}\right)^{k}\left(1-\frac{|A|}{|B|}\right)^{n-k},
$$

where $|A|$ represents the Lebesgue measure of the set $|A|$. We observe that the random variables $\Phi(A)$ and $\Phi(B)$ are not independent even if $A \cap B=\emptyset$, which makes the analysis of the interference and related quantities difficult.

We now describe the Poisson point process which exhibits better independence properties than the BPP and is obtained as a limit of the BPP, by increasing $|B| \rightarrow \infty$ while keeping $n^{-1}|B|$ constant.

\section{A.1.2 Poisson Point Process (PPP)}

The PPP is perhaps the most well-studied point process, and its importance stems from its ease of analysis. A stationary PPP of density 
(or intensity) $\lambda$ is characterized by the following two properties:

- The number of points in any set $B \subset \mathbb{R}^{d}$ is a Poisson random variable with mean $\lambda|B|$.

- The number of points in disjoint sets are independent random variables.

From the definition we observe that

$$
\mathbb{P}(\Phi(B)=k)=\exp (-\lambda|B|) \frac{(\lambda|B|)^{k}}{k !},
$$

and in particular the void probability is given by $\exp (-\lambda|B|)$. Some interesting properties of the PPP are the following:

- The superposition of two PPPs of densities $\lambda_{1}$ and $\lambda_{2}$ results in a PPP of density $\lambda_{1}+\lambda_{2}$.

- The thinning of a PPP (i.e., selecting a point of the process with probability $p$ independently of the other points and discard it with probability $1-p$ ) results in two independent PPPs of intensity measures $p \lambda$ and $(1-p) \lambda$. For example, using ALOHA as the MAC protocol in a wireless network leads to a thinning of the node set, and when the underlying nodes form a PPP, the resulting transmitter and receiver node locations also form a PPP.

- Conditioned on the number of points of $\Phi$ in a compact set $B \subset \mathbb{R}^{d}$, the set of points $\Phi \cap B$ form a BPP. This fact can be used for the simulation of a PPP.

An inhomogeneous PPP of intensity measure $\Lambda$ is defined in a similar manner as the stationary PPP, except that the number of points in a set $B$ is a Poisson random variable with mean $\Lambda(B)$. For example using $\Lambda(B)=(2 \pi)^{-1} \int_{B} \exp \left(-\|x\|^{2} / 2\right) \mathrm{d} x$ results in a PPP with Gaussian density. Another example is the finite PPP: A PPP with density $\lambda$, restricted to a bounded domain $A$, results in an inhomogeneous PPP with $\Lambda(B)=\lambda|A \cap B|$. Observe that a stationary PPP is a special case of the inhomogeneous PPP with $\Lambda(B)=\lambda|B|$.

The independence properties of a PPP are retained if each point of the process is subject to an independent random operation. Indepen- 
dent thinning of the point process is one such operation and as mentioned earlier results in two independent PPPs. The following mapping theorem illustrates the effect of applying a function $f$ to each point of a PPP.

Theorem A.1 (Mapping theorem). Let $\Phi$ be an inhomogeneous $\mathrm{PPP}$ on $\mathbb{R}^{d}$ with intensity function $\Lambda$, and let $f: \mathbb{R}^{d} \rightarrow \mathbb{R}^{s}$ be measurable and $\Lambda\left(f^{-1}\{y\}\right)=0$ for all $y \in \mathbb{R}^{s}$. Assume further that

$$
\mu(B)=\Lambda\left(f^{-1}(B)\right)
$$

satisfies $\mu(B)<\infty$ for all bounded $B$. Then $f(\Phi)$ is a non-homogeneous PPP on $\mathbb{R}^{s}$ with intensity measure $\mu$.

From the above theorem, we observe that if $\Phi$ is a stationary PPP of intensity $\lambda$ and $\mathbf{A}$ is a nonsingular linear mapping, then $\mathbf{A} \Phi=\{\mathbf{A} x$ : $x \in \Phi\}$ is also a stationary $\mathrm{PPP}$ with intensity $\lambda \operatorname{det}\left(\mathbf{A}^{-1}\right)$.

\section{A.1.3 Poisson Cluster Process (PCP)}

A PCP consists of a parent PPP $\Phi_{p}=\left\{\mathrm{x}_{1}, \mathrm{x}_{2}, \ldots\right\}$ of density $\lambda_{p}$. The clusters are of the form $N_{\mathrm{x}_{i}}=N_{i}+\mathrm{x}_{i}$ for each $\mathrm{x}_{i} \in \Phi_{p}$. The $N_{i}$ are a family of identical, independently distributed point sets and also independent of the parent process. The complete process $\Phi$ is given by

$$
\Phi=\bigcup_{x \in \Phi_{p}} N_{x} .
$$

The daughter points of the representative cluster $N_{o}$ are scattered independently and with an identical spatial distribution

$$
F_{\mathrm{cl}}(A)=\int_{A} f_{\mathrm{cl}}(x) \mathrm{d} x, \quad A \subset \mathbb{R}^{2},
$$

around the origin. The number of points in a cluster may be random, and we denote its mean by $\bar{c}$.

We now provide some basic definitions and intensity measures associated with point processes. 
Definition A.2. A point process $\Phi=\left\{x_{n}\right\}$ is said to be stationary if

$$
\mathbb{P}(\Phi \in Y)=\mathbb{P}\left(\Phi_{x} \in Y\right)
$$

for all $Y \in \mathcal{N}$, where $\Phi_{x}=\left\{x_{n}+x\right\}$. A point process $\Phi$ is said to be isotropic if

$$
\mathbb{P}(\Phi \in Y)=\mathbb{P}\left(\Phi_{x} \in \mathrm{r} Y\right),
$$

where $r$ is a rotation in $\mathbb{R}^{d}$.

A point process that is both stationary and isotropic is said to be motion-invariant. From the above definition we observe that a BPP is not a stationary point process, and that it is isotropic if the domain is isotropic. A PPP with constant intensity measure, i.e., with measure proportional to the Lebesgue measure, is stationary and isotropic, and the proportionality constant is the intensity. We now define an equivalent of the mean of random variables for the point processes.

Definition A.3. The intensity measure of a point process $\Phi$ is equal to the average number of points in a set $B \subset \mathbb{R}^{d}$, i.e.,

$$
\Lambda(B)=\mathbb{E}(\Phi(B)) .
$$

If $\Phi$ is stationary, then $\Lambda(B)=\lambda|B|$ where $\lambda$ is called the intensity (density) of the stationary point process $\Phi$. We have

- The intensity measure $\Lambda(B)$ of a BPP is equal to $\frac{n|B \cap A|}{|A|}$.

- For a stationary PPP, the intensity measure is equal to $\lambda|B|$.

- For a PCP, the intensity measure is equal to $\lambda_{p} \bar{c}|B|$.

It is often necessary to evaluate the average sum of a function evaluated at the point of the process $\Phi$. For example when $\ell(x)$ represents the path loss model, the average interference seen at the origin when the transmitting nodes form a point process $\Phi$ is given by $\mathbb{E} \sum_{\mathbf{x} \in \Phi} \ell(\mathrm{x})$. The next theorem helps in evaluating such sums. 
Theorem A.2. Campbell's Theorem: Let $f(x): \mathbb{R}^{d} \rightarrow[0, \infty]$ be a measurable function. Then

$$
\mathbb{E}\left(\sum_{\mathrm{x} \in \Phi} f(\mathrm{x})\right)=\int_{\mathbb{R}^{d}} f(x) \Lambda(\mathrm{d} x) .
$$

Proof. Interpreting $\Phi$ as the random measure (A.1), the sum of $f(x)$ over the point process $\Phi$ is

$$
\sum_{\mathrm{x} \in \Phi} f(\mathrm{x})=\int_{\mathbb{R}^{d}} f(x) \Phi(\mathrm{d} x) .
$$

Taking the average on both sides we have

$$
\begin{aligned}
\mathbb{E}\left[\sum_{\mathrm{x} \in \Phi} f(\mathrm{x})\right] & =\mathbb{E}\left[\int_{\mathbb{R}^{d}} f(x) \Phi(\mathrm{d} x)\right] \\
& \stackrel{(a)}{=} \int_{\mathrm{N}} \int_{\mathbb{R}^{d}} f(x) \phi(\mathrm{d} x) \mathbf{P}(\mathrm{d} \phi) \\
& \stackrel{(b)}{=} \int_{\mathbb{R}^{d}} f(x) \int_{\mathrm{N}} \phi(\mathrm{d} x) \mathbf{P}(\mathrm{d} \phi) \\
& \stackrel{(c)}{=} \int_{\mathbb{R}^{d}} f(x) \mathbb{E}[\Phi(\mathrm{d} x)] \\
& \stackrel{(d)}{=} \int_{\mathbb{R}^{d}} f(x) \Lambda(\mathrm{d} x) .
\end{aligned}
$$

In (a) the average is written as an integral over the set of point sequences and the distribution measure $\mathbf{P}$. (b) results by interchanging the integrals (which is essentially an application of Fubini's theorem), (c) follows by rewriting the inner integral as an expectation operator (inverse step of $(a)$ ) and $(d)$ follows from the definition of the intensity measure.

When $\Phi$ is stationary with intensity $\lambda$ the right side is equal to $\lambda \int_{\mathbb{R}^{d}} f(x) \mathrm{d} x$. Hence in a wireless network the mean interference seen at the origin, when the transmitters have density $\lambda$, is $\lambda \int_{\mathbb{R}^{d}} \ell(x) \mathrm{d} x$. We also observe that the average sum depends only on the (firstorder) intensity of the process, and hence any two stationary node 
distributions with the same intensity measure lead to equal average interference.

Definition A.4. The second-order product density $\rho^{(2)}$ of a point process is defined by the following relation:

$$
\mathbb{E}\left(\sum_{\mathrm{x}_{1}, \mathrm{x}_{2} \in \Phi}^{\neq} f\left(\mathrm{x}_{1}, \mathrm{x}_{2}\right)\right)=\int_{\mathbb{R}^{d}} \int_{\mathbb{R}^{d}} f\left(x_{1}, x_{2}\right) \rho^{(2)}\left(x_{1}, x_{2}\right) \mathrm{d} x_{1} \mathrm{~d} x_{2}
$$

for any non-negative and measurable function $f$.

$\sum_{x, y}^{f}$ represents summation only over distinct $x$ and $y$. When $\Phi$ is stationary, $\rho^{(2)}\left(x_{1}, x_{2}\right)$ depends only on $x_{1}-x_{2}$. $\rho^{(2)}\left(x_{1}, x_{2}\right) \mathrm{d} x_{1} \mathrm{~d} x_{2}$ can be interpreted as the probability that there exist two points of $\Phi$ in the infinitesimal regions $\mathrm{d} x_{1}$ and $\mathrm{d} x_{2} \cdot \rho^{(2)}\left(x_{1}, x_{2}\right)$ can be used to evaluate the second-order properties of the interference $I$. We have

$$
\begin{aligned}
\mathbb{E}\left[I^{2}\right]= & \mathbb{E}\left[\sum_{\mathrm{x} \in \Phi} \ell(\mathrm{x})^{2}\right]+\mathbb{E}\left[\sum_{\mathrm{x}_{1}, \mathrm{x}_{2} \in \Phi}^{\neq} \ell\left(\mathrm{x}_{1}\right) \ell\left(\mathrm{x}_{2}\right)\right] \\
= & \int_{\mathbb{R}^{d}} \ell(x)^{2} \Lambda(\mathrm{d} x) \\
& +\int_{\mathbb{R}^{d}} \int_{\mathbb{R}^{d}} \ell\left(x_{1}\right) \ell\left(x_{2}\right) \rho^{(2)}\left(x_{1}, x_{2}\right) \mathrm{d} x_{1} \mathrm{~d} x_{2} .
\end{aligned}
$$

For a stationary PPP of density $\lambda$, we have the following lemma for the mean and the variance which follow from Campbell's theorem and the second-order product density.

Lemma A.3. For a PPP of density $\lambda$, the mean of the sum $\sum_{\mathrm{x} \in \Phi} f(\mathrm{x})$ is

$$
\mathbb{E}\left[\sum_{\mathrm{x} \in \Phi} f(\mathrm{x})\right]=\lambda \int_{\mathbb{R}^{d}} f(x) \mathrm{d} x
$$

and the variance is

$$
\operatorname{var}\left[\sum_{\mathrm{x} \in \Phi} f(\mathrm{x})\right]=\lambda \int_{\mathbb{R}^{d}} f(x)^{2} \mathrm{~d} x .
$$


Proof. Follows from Campbell's theorem and the fact that $\rho^{(2)}\left(x_{1}, x_{2}\right)=\lambda^{2}$ for a PPP of density $\lambda$.

We now provide an equivalent of the moment generating functional for the point process.

Definition A.5 (Probability generating functional (PGFL)). Let $\nu(x): \mathbb{R}^{d} \rightarrow[0, \infty)$ be measurable. The PGFL of the point process $\Phi$ is defined as

$$
\mathcal{G}[\nu]=\mathbb{E} \prod_{\mathbf{x} \in \Phi} \nu(\mathbf{x})
$$

Observe that the PGFL is a functional, i.e., acts on a function and when the function is a multivariate, a dot "." is used to represent the variable that the PGFL acts on. For example $\mathcal{G}[v(\cdot+y)]=\mathbb{E} \prod_{\mathrm{x} \in \Phi} v(\mathrm{x}+y)$.

The probability generating functional of a BPP is given by

$$
\mathcal{G}[\nu]=\left(\frac{1}{|A|} \int_{\mathbb{R}^{d}} \nu(x) \mathrm{d} x\right)^{n} .
$$

For a PPP it is equal to

$$
\mathcal{G}[\nu]=\exp \left(-\int_{\mathbb{R}^{d}}(1-\nu(x)) \Lambda(\mathrm{d} x)\right) .
$$

The probability generating functional of the PCP is given by

$$
\mathcal{G}[\nu]=\exp \left(-\lambda_{p} \int_{\mathbb{R}^{d}} 1-M\left(\int_{\mathbb{R}^{d}} \nu(x+y) f_{\mathrm{cl}}(x) \mathrm{d} x\right)\right),
$$

where $M(z)$ is the moment generating function of the number of points in the representative cluster.

A PGFL completely characterizes a simple point process and can be used to derive all the density measures of a point process. A PGFL is very useful to evaluate the Laplace transform of the sum $\sum_{\mathrm{x} \in \Phi} f(\mathrm{x})$. We have

$$
\begin{aligned}
\mathbb{E} \exp \left(-s \sum_{\mathrm{x} \in \Phi} f(\mathrm{x})\right) & =\mathbb{E} \prod_{\mathrm{x} \in \Phi} \exp (-s f(\mathrm{x})) \\
& =\mathcal{G}[\exp (-s f(\cdot))]
\end{aligned}
$$


In a wireless network the above procedure can be used to derive the Laplace transform of the interference. Another simple application of the PGFL is the derivation of the first-contact distribution of a stationary point process, which is equal to the distribution of the nearest point distance of the process from the origin, i.e.,

$$
\begin{aligned}
D(r) & =\mathbb{P}(\Phi(b(o, r))=0) \\
& =\mathbb{E} \prod_{\mathrm{x} \in \Phi} 1-\mathbf{1}_{b(o, r)}(\mathrm{x}) \\
& =\mathcal{G}\left[\mathbf{1}_{b(o, r)^{c}}(x)\right] .
\end{aligned}
$$

In general, Campbell's theorem is used to evaluate the average of a sum and the PGFL for the average of a product of a function over the point process.

In the next section, we provide the equivalent of conditional probability for the point process.

\section{A.2 Palm Distributions}

Palm distributions are the counterparts to the conditional distributions for the point processes, and they arise when the point process is conditioned to have a point at $x \in \mathbb{R}^{d}$. The use of Palm measures arises in a wireless network when we calculate outage probabilities which requires conditioning on either the receiver or the transmitter location. We provide the definition of Palm distribution in terms of the Campbell measure, which is a measure on $\mathbb{R}^{d} \times \mathrm{N}$.

Definition A.6. The reduced Campbell measure of a point process is defined as

$$
C^{!}(A \times Y)=\mathbb{E}\left[\sum_{\mathrm{x} \in \Phi \cap A} \mathbf{1}(\Phi \backslash\{\mathrm{x}\} \in Y)\right]
$$

for any Borel set $A \subset \mathbb{R}^{d}$ and $Y \in \mathcal{N}$. 
An immediate consequence of this definition is the following theorem:

Theorem A.4 (Mecke). Let $f(x, \phi)$ be a measurable function on $\mathbb{R}^{d} \times \mathrm{N}$. Then,

$$
\mathbb{E} \sum_{\mathrm{x} \in \Phi} f(\mathrm{x}, \Phi \backslash\{\mathrm{x}\})=\int_{\mathbb{R}^{d}} \int_{\mathrm{N}} f(x, \phi) \mathrm{d} C^{!}(x, \phi) .
$$

If $C(. \times Y)$ is absolutely continuous with respect to the intensity measure $\Lambda$, we have by the Radon-Nikodym theorem

$$
C^{!}(A \times Y)=\int_{\mathbb{R}^{d}} \mathbf{P}^{! x}(Y) \mathrm{d} \Lambda(x) .
$$

$\mathbf{P}^{! x}$ is called the reduced Palm measure of the process $\Phi$. Intuitively, this is equal to conditioning on the point process having a point at $x$ but not counting it. From (A.6), Mecke's theorem, and the definition of the Campbell measure, we have

$$
\mathbb{E} \sum_{\mathrm{x} \in \Phi} f(\mathrm{x}, \Phi \backslash\{\mathrm{x}\})=\int_{\mathbb{R}^{d}} \mathbb{E}^{! x}(f(x, \Phi)) \Lambda(\mathrm{d} x) .
$$

We now provide a brief description of the reduced Palm probability measure for PPP and PCP conditioned on a point being at the origin. The following theorem, often referred to as Slivnyak's theorem, is in this generality actually due to Mecke [34].

Theorem A.5 (Mecke; Slivnyak). For a PPP,

$$
\mathbf{P}^{! o} \equiv \mathbf{P}
$$

i.e., the reduced Palm distribution equals the distribution of the PPP itself.

This is also a complete characterization of the PPP. It says that an additional point at $o$ does not change the distribution of the other points of the PPP. Hence for a stationary PPP, Mecke's theorem Theorem A.4 reads

$$
\mathbb{E} \sum_{\mathrm{x} \in \Phi} f(\mathrm{x}, \Phi \backslash\{\mathrm{x}\})=\lambda \int_{\mathbb{R}^{d}} \mathbb{E} f(x, \Phi) \mathrm{d} x
$$


As an application we calculate the average number of transmitters that can connect to a receiver in the presence of interference. Suppose the transmitters form a PPP $\Phi$ of intensity $\lambda$ on the plane and a receiver is located at the origin. A transmitter $x$ can connect to the receiver at $o$ if the signal-to-interference ratio $\operatorname{SIR}_{\phi \backslash\{x\}}(o, x)>\beta$, where $\operatorname{SIR}_{\psi}(o, x)=$ $\ell(x) / \sum_{y \in \psi} \ell(y)$. Let $\eta$ denote the average number of transmitters that connect to the receiver at $o$. We then have

$$
\begin{aligned}
\eta & =\mathbb{E} \sum_{x \in \Phi} \mathbf{1}\left(\operatorname{SIR}_{\Phi \backslash\{x\}}(o, x)>\beta\right) \\
& =\lambda \int_{\mathbb{R}^{d}} \mathbb{E} \mathbf{1}\left(\operatorname{SIR}_{\Phi}(o, x)>\beta\right) \mathrm{d} x \\
& =\lambda \int_{\mathbb{R}^{d}} \mathbb{P}\left(\operatorname{SIR}_{\Phi}(o, x)>\beta\right) \mathrm{d} x .
\end{aligned}
$$

So the calculation of $\eta$ is reduced to the problem of deducing the link formation probability of a pair of nodes.

Next we provide a characterization of the Palm measure for a PCP.

Theorem A.6. The Palm measure of a PCP is given by

$$
\mathbf{P}^{! o}=\mathbf{P} * \Omega^{! o}
$$

where $\Omega^{! o}$ is the reduced Palm measure of the representative cluster $N_{o}$, given by

$$
\Omega^{! o}(Y)=\frac{1}{\bar{c}} \mathbb{E} \sum_{\mathrm{x} \in N_{o}} \mathbf{1}_{Y}\left(\left(N_{o}-\mathrm{x}\right) \backslash\{o\}\right) .
$$

* denotes the convolution of the distributions, which corresponds to the superposition of the two point measures.

Definition A.7. For a point process $\Phi$, the second-order moment measure is defined as

$$
\mathcal{K}_{2}(B)=\mathbb{E}^{! o} \sum_{\mathrm{x} \in \Phi} \mathbf{1}_{B}(\mathrm{x})
$$

for any Borel set $B \subset \mathbb{R}^{d}$. 
It is equal to the average number of points in the set $B$ given that there is a point at the origin but without counting the point. We also have the following relation between the second-order moment measure and the second-order product measure

$$
\lambda^{2} \mathcal{K}_{2}(B)=\int_{B} \rho^{2}(x) \mathrm{d} x .
$$

An important characteristic of a stationary point process is Ripley's $K$-function defined as $K(r)=\mathcal{K}_{2}(b(o, r))$. For a $d$-dimensional PPP, it is $c_{d} r^{d}$, where $c_{d}$ is equal to the volume of the unit ball in $d$-dimensions. For any stationary point process $K(r) \sim c_{d} r^{d}, r \rightarrow \infty$.

Similar to the reduced second moment measure, the reduced $n$-th factorial moment measure $[8,45]$ of a point process $\Phi$, is defined as

$$
\lambda^{n-1} \mathcal{K}_{n}(B) \triangleq \mathbb{E}^{! o}\left[\sum_{\mathrm{x}_{1}, \ldots, \mathrm{x}_{n-1} \in \Phi}^{\mathrm{x}_{i} \neq \mathrm{x}_{j}} 1_{B}\left(\mathrm{x}_{1}, \ldots, \mathrm{x}_{n-1}\right)\right],
$$

where $B=B_{1} \times \ldots \times B_{n-1}, B_{i} \subset \mathbb{R}^{2}$. When $\mathcal{K}_{n}(B)$ is absolutely continuous with respect to the Lebesgue measure, the $n$-th order product density $\rho^{(n)}$ exists, and we can write (in the stationary case) $[23,45]$

$$
\mathcal{K}_{n}(B)=\frac{1}{\lambda^{n}} \int_{B} \rho^{(n)}\left(x_{1}, x_{2}, \ldots, x_{n-1}\right) \mathrm{d} x .
$$

One of the fundamental distance distributions of a point process is the nearest-neighbor distribution. For a stationary process, we condition on the fact that there is a point at the origin and find the distance distribution of the nearest neighbor as follows:

$$
\begin{aligned}
F_{N}(r)= & \mathbb{P}(\text { the nearest-neighbor distance of } \\
& \text { the point at the origin is less than } r) \\
= & 1-\mathbb{P}^{! o}(\Phi(b(o, r))=0)
\end{aligned}
$$

The nearest-neighbor distribution which depends on the reduced Palm measure is in general different from the first-contact distribution $D(r)$ (A.5). Indeed the metric $J(r)=\left(1-F_{N}(r)\right) /(1-D(r))$, called the J-function, is used to distinguish between different point processes. For a $\mathrm{PPP}$, since $\mathbf{P}^{! o}=\mathbf{P}$ we have $F_{N}(r)=1-\exp (-\Lambda(b(o, r)))$ which 
is equal to the first-contact distribution and hence $J(r)=1$. The $n$-th nearest-neighbor distributions of the PPP are provided in [19].

Similar to the definition of the PGFL, the conditional PGFL is defined as:

Definition A.8 (Conditional PGFL). Let $v(x): \mathbb{R}^{d} \rightarrow(0, \infty)$. The conditional PGFL is

$$
\tilde{\mathcal{G}}[v]=\mathbb{E}^{! o}\left[\prod_{x \in \Phi} v(x)\right]
$$

For a PPP, by Slivnyak's theorem the conditional PGFL is equal to the PGFL, i.e.,

$$
\tilde{\mathcal{G}}[v]=\exp \left(-\int_{\mathbb{R}^{d}}(1-v(x)) \Lambda(\mathrm{d} x)\right)
$$

\section{A.3 Stable Distributions}

The interference in a wireless network follows a heavy-tailed distribution when the path loss model is given by $\ell(x)=\|x\|^{-\alpha}, \alpha>d$, and it follows a stable distribution when the transmitting nodes form a PPP. In this section, we introduce stable distributions and their important properties. A random variable $\mathbf{X}$ is stable if for some $a, b, c \in(0, \infty)$, $d \in \mathbb{R}$,

$$
a \mathbf{X}_{1}+b \mathbf{X}_{2}=c \mathbf{X}+d
$$

where $\mathbf{X}_{1}$ and $\mathbf{X}_{2}$ are some independent copies of $\mathbf{X}$. The only known stable distributions with closed-form density functions are the Gaussian, the Lévy, and the Cauchy distribution.

Theorem A.7. For any stable random variable $\mathbf{X}$, there is a $0<\delta \leq 2$ such that the number $c$ in the definition satisfies

$$
a^{\delta}+b^{\delta}=c^{\delta} .
$$

$\delta$ is called the characteristic exponent of the stable random variable. 
A stable distribution can be characterized by its characteristic function:

$$
\mathbb{E}\left[e^{j t \mathbf{X}}\right]= \begin{cases}\exp \left(j t \mu-\gamma|t|^{\delta}(1-j \beta \operatorname{sgn}(t) \tan (\pi \delta / 2))\right) & \delta \neq 1 \\ \exp \left(j t \mu-\gamma|t|\left(1+j \frac{2 \beta}{\pi} \operatorname{sgn}(t) \log (|t|)\right)\right) & \delta=1,\end{cases}
$$

where $\operatorname{sgn}(t)$ is the sign of $t . \beta \in[-1,1]$ is the skew parameter, $\mu$ is the drift, and $\gamma$ is the dispersion parameter. Alternatively, a stable random variable with $\delta<1$ and $\beta=1$ can be described by its Laplace transform, which is given by

$$
\mathbb{E}\left[e^{-s \mathbf{X}}\right]=\exp \left(\frac{-\gamma}{\cos (\pi \delta / 2)} s^{\delta}\right) .
$$

Except for the Gaussian $(\delta=2)$, all the stable distributions are heavytailed with parameter $\delta$, i.e.,

$$
\lim _{t \rightarrow \infty} t^{\delta} \mathbb{P}(\mathbf{X}>t)=C, 0 \leq \delta<2 .
$$

The $p$-th moment of a stable random variable with $\delta<2$ is finite if and only if $p<\delta$. For a stable random variable with drift 0 and skew 1 , the $p$-th moment is given by

$$
\mathbb{E}|\mathbf{X}|^{p}=\frac{(\sec (\delta \pi / 2))^{p / \delta} \Gamma(1-p / \delta)}{\Gamma(1-p)} \gamma^{\delta / p}, \quad p<\delta, \delta \neq 1,
$$

where $\Gamma(x)$ is the standard gamma function. 


\section{Acknowledgments}

The authors would like to thank François Baccelli and Steven Weber for their valuable comments on the manuscript.

The partial support of the U.S. National Science Foundation and the DARPA/IPTO IT-MANET program is gratefully acknowledged. 


\section{Notations and Acronyms}

\section{Notation and Symbols:}

$\begin{array}{ll}\text { Symbol } & \text { Definition/explanation } \\ \mathbb{Z}, \mathbb{N} & \text { integers, positive integers } \\ \mathbb{R}, \mathbb{R}^{+} & \text {real numbers, positive real numbers } \\ \mathbb{C} & \text { complex numbers } \\ {[k]} & \text { the set }\{1,2, \ldots, k\} \\ j & \sqrt{-1} \\ \mathbf{1}_{A}(x) & \text { indicator function } \\ \mathbf{1}_{x>b}=\mathbf{1}(x>b) & \text { shortcut for } \mathbf{1}_{\{x: x>b\}}(x) \\ u(x) & \triangleq \mathbf{1}_{x \geq 0} \text { unit step function) } \\ d & \text { number of dimensions of the network } \\ \# A & \text { cardinality of } A \\ \mathbb{P}(A) & \text { probability of event } A \\ \mathbb{E}(X) & \text { expectation of random variable } X \\ \mathcal{L} & \text { Laplace transform of random variable } X \\ |\cdot| & \text { Lebesgue measure } \\ o & \text { origin in } \mathbb{R}^{d} \\ B & \text { a Borel subset of } \mathbb{R}^{d}\end{array}$


244 Notations and Acronyms

$\begin{array}{ll}B^{c} & \mathbb{R}^{d} \backslash B \\ b(x, r) & \left\{y \in \mathbb{R}^{d}:\|y-x\|<r\right\} \\ & (\text { ball of radius } r \text { centered at } x) \\ c_{d} \triangleq|b(o, 1)| & =\pi^{d / 2} / \Gamma(1+d / 2) \\ & (\text { volume of the } d \text {-dim. unit ball) } \\ F_{X}(x)=\mathbb{P}(X \leq x) & \text { distribution of random variable } X(\mathrm{CDF}) \\ \Phi=\left\{\mathrm{x}_{i}\right\} \subset \mathbb{R}^{d} & \text { point process in } d \text { dimensions } \\ \Lambda, \lambda & \text { counting measure and density for } \Phi \\ h & \text { (power) fading random variable }(\mathbb{E}(h)=1) \\ \theta \in \mathbb{R}^{+} & \text {min. SIR or SINR for successful communication } \\ \ell: \mathbb{R}^{d} \rightarrow \mathbb{R}^{+} & \text {(isotropic) large-scale path loss function } \\ \alpha & \text { path loss exponent } \\ \delta \triangleq d / \alpha & \text { ratio of number of dim. to path loss exponent } \\ W & \text { thermal noise } \\ \mathrm{N}, \mathcal{N} & \text { space of simple sequences, its } \sigma \text {-algebra }\end{array}$

\section{Acronyms:}

$\begin{array}{ll}\text { LHS } & \text { left-hand side (of equation) } \\ \text { RHS } & \text { right-hand side (of equation) } \\ \text { a.s. } & \text { almost surely (with probability 1) } \\ \text { iid } & \text { independent and identically distributed } \\ \text { PPP } & \text { Poisson point process } \\ \text { PDF } & \text { Probability density function } \\ \text { CDF } & \text { Cumulative distribution function } \\ \text { CCDF } & \text { Complementary CDF } \\ \text { PGFL } & \text { Probability-generating functional }\end{array}$




\section{References}

[1] J. G. Andrews, S. Weber, and M. Haenggi, "Ad hoc networks: To spread or not to spread?," IEEE Communications Magazine, vol. 45, pp. 84-91, December 2007.

[2] J. C. Arnback and W. van Blitterswijk, "Capacity of slotted ALOHA in rayleigh-fading channels," IEEE Journal on Selected Areas in Communications, vol. SAC-5, pp. 261-269, February 1987.

[3] F. Baccelli, B. Blaszczyszyn, and P. Mühlethaler, "An Aloha protocol for multihop mobile wireless networks," IEEE Transactions on Information Theory, vol. 52, pp. 421-436, February 2006.

[4] F. Baccelli, B. Blaszczyszyn, and P. Mühlethaler, "Stochastic analysis of spatial and opportunistic Aloha," IEEE Journal on Selected Areas in Communications, vol. 27, pp. 1105-1119, September 2009.

[5] N. H. Bingham, C. M. Goldie, and J. L. Teugels, Regular Variation. Cambridge University Press, 1989.

[6] N. Campbell, "Discontinuities in light emission," Mathematical Proceedings of the Cambridge Philosophical Society, vol. 15, pp. 310-328, 1909.

[7] N. Campbell, "The study of discontinuous phenomena," Mathematical Proceedings of the Cambridge Philosophical Society, vol. 15, pp. 117-136, 1909.

[8] D. J. Daley and D. Vere-Jones, An Introduction to the Theory of Point Processes: Volume II: General Theory and Structure. Springer, second edition, 2007.

[9] O. Dousse and P. Thiran, "Connectivity vs Capacity in Dense Ad Hoc Networks," in IEEE INFOCOM, Hong Kong, March 2004.

[10] W. Feller, An Introduction to Probability Theory and its Applications, Vol. 2. Wiley, second edition, 1970. 
[11] M. Franceschetti, J. Bruck, and L. Schulman, "A random walk model of wave propagation," IEEE Transactions on Antennas and Propagation, vol. 52, pp. 1304-1317, May 2004.

[12] R. K. Ganti and M. Haenggi, "Interference in ad hoc networks with general motion-invariant node distributions," in 2008 IEEE International Symposium on Information Theory (ISIT'08), Toronto, Canada, July 2008.

[13] R. K. Ganti and M. Haenggi, "Interference and outage in clustered wireless ad hoc networks," IEEE Transactions on Information Theory, vol. 55, pp. 40674086, September 2009.

[14] R. K. Ganti and M. Haenggi, "Spatial and temporal correlation of the interference in ALOHA Ad Hoc networks," IEEE Communications Letters, vol. 13, pp. 631-633, September 2009.

[15] E. N. Gilbert and H. O. Pollak, "Amplitude distribution of shot noise," Bell Systems Technical Journal, vol. 39, pp. 333-350, March 1960.

[16] M. Grossglauser and D. Tse, "Mobility increases the capacity of ad hoc wireless networks," IEEE/ACM Transactions on Networking, vol. 10, pp. 477-486, August 2002.

[17] P. Gupta and P. R. Kumar, "The capacity of wireless networks," IEEE Transactions on Information Theory, vol. 46, pp. 388-404, March 2000.

[18] A. Gut, Probability: A Graduate Course, Springer Texts in Statistics. Springer, 2005.

[19] M. Haenggi, "On distances in uniformly random networks," IEEE Transactions on Information Theory, vol. 51, pp. 3584-3586, October 2005.

[20] M. Haenggi, "A geometric interpretation of fading in wireless networks: Theory and applications," IEEE Transactions on Information Theory, vol. 54, pp. 5500-5510, December 2008.

[21] M. Haenggi, "Outage, local throughput, and capacity of random wireless networks," IEEE Transactions on Wireless Communications, vol. 8, pp. 43504359, August 2009.

[22] K. Hamdi, "Exact probability of error of BPSK communication links subjected to asynchronous interference in Rayleigh fading environment," IEEE Transactions on Communications, vol. 50, no. 10, pp. 1577-1579, 2002.

[23] K.-H. Hanisch, "Reduction of $n$-th moment measures and the special case of the third moment measure of stationary and isotropic planar point processes," Mathematische Operationsforschung und Statistik, Serie Statistik, vol. 14, no. 3, pp. 421-435, 1983.

[24] A. Hasan and J. Andrews, "The guard zone in wireless ad hoc networks," IEEE Transactions on Wireless Communications, vol. 6, pp. 897-906, March 2007.

[25] L. Heinrich and V. Schmidt, "Normal convergence of multidimensional shot noise and rates of this convergence," Advances in Applied Probability, vol. 17, no. 4, pp. 709-730, 1985.

[26] J. Ilow and D. Hatzinakos, "Analytical alpha-stable noise modeling in a poisson field of interferers or scatterers," IEEE Transactions on Signal Processing, vol. 46, no. 6, pp. 1601-1611, 1998.

[27] H. Inaltekin, M. Chiang, H. V. Poor, and S. B. Wicker, "On unbounded path-loss models: effect of singularity on wireless network performance," IEEE 
Journal on Selected Areas in Communications, vol. 27, pp. 1078-1092, September 2009 .

[28] N. Jindal, S. Weber, and J. Andrews, "Fractional power control for decentralized wireless networks," IEEE Transactions on Wireless Communications, vol. 7, pp. 5482-5492, December 2008.

[29] O. Kallenberg, Foundations of Modern Probability. Springer, second edition, 2001.

[30] J. F. C. Kingman, Poisson Processes. Oxford Science Publications, 1993.

[31] J.-P. M. G. Linnartz, "Exact analysis of the outage probability in multiple-user radio," IEEE Transactions on Communications, vol. 40, pp. 20-23, January 1992.

[32] S. B. Lowen and M. C. Teich, "Power-law shot noise," IEEE Transactions on Information Theory, vol. 36, pp. 1302-1318, November 1990.

[33] R. Mathar and J. Mattfeldt, "On the distribution of cumulated interference power in Rayleigh fading channels," Wireless Networks, vol. 1, pp. 31-36, February 1995.

[34] J. Mecke, "Eine charakteristische Eigenschaft der doppelt stochastischen Poissonschen Prozesse," Zeitschrift für Wahrscheinlichkeitstheorie und Verwandte Gebiete, vol. 11, pp. 74-81, 1968.

[35] S. Nadarajah and S. Kotz, "On the product and ratio of gamma and weibull random variables," Econometric Theory, vol. 22, no. 02, pp. 338-344, 2006.

[36] K. Nakagawa, "Application of Tauberian theorem to the exponential decay of the tail probability of a random variable," IEEE Transactions on Information Theory, vol. 53, pp. 3239-3249, September 2007.

[37] H. Q. Nguyen, F. Baccelli, and D. Kofman, "A stochastic geometry analysis of dense 802.11 networks," in IEEE INFOCOM, Anchorage, AK, May 2007.

[38] P. Patel and J. Holtzman, "Analysis of a simple successive interference cancellation scheme in a DS/CDMA system," IEEE Journal on Selected Areas in Communications, vol. 12, no. 5, pp. 796-807, 1994.

[39] S. O. Rice, "Mathematical analysis of random noise," Bell System Technical Journal, vol. 23, pp. 282-332, July 1944.

[40] G. Samorodnitsky and M. S. Taqqu, Stable Non-Gaussian Random Processes: Stochastic Models with Infinite Variance. Chapman \& Hall, Jan 1994.

[41] W. Schottky, "Über spontane Stromschwankungen in verschiedenen Elektrizitätsleitern," Annalen der Physik, vol. 57, pp. 541-567, 1918.

[42] X. Shang, G. Kramer, and B. Chen, "A new outer bound and the noisyinterference sum-rate capacity for Gaussian interference channels," IEEE Transactions on Information Theory, vol. 55, pp. 689-699, February 2009.

[43] E. S. Sousa and J. A. Silvester, "Optimum transmission ranges in a directsequence spread-spectrum multihop packet radio network," IEEE Journal on Selected Areas in Communications, vol. 8, pp. 762-771, June 1990.

[44] K. Stamatiou, F. Rossetto, M. Haenggi, T. Javidi, J. R. Zeidler, and M. Zorzi, "A delay-minimizing routing strategy for wireless multihop networks," in 2009 Workshop on Spatial Stochastic Models for Wireless Networks (SpaSWiN'09), Seoul, Korea, June 2009.

[45] D. Stoyan, W. S. Kendall, and J. Mecke, Stochastic Geometry and its Applications. John Wiley \& Sons, second edition, 1995. 
[46] H. Takagi and L. Kleinrock, "Optimal transmission ranges for randomly distributed packet radio terminals," IEEE Transactions on Communications, vol. COM-32, pp. 246-257, March 1984.

[47] A. Viterbi, "Very low-rate convolutional codes for maximum theoretical performance of spread-spectrum multiple-access channels," IEEE Journal on Selected Areas in Communications, vol. 8, no. 4, pp. 641-649, 1990.

[48] S. Weber, J. Andrews, X. Yang, and G. D. Veciana, "Transmission capacity of wireless ad hoc networks with successive interference cancellation," IEEE Transactions on Information Theory, vol. 53, pp. 2799-2814, August 2007.

[49] S. Weber, J. G. Andrews, and N. Jindal, "The effect of fading, channel inversion, and threshold scheduling on ad hoc networks," IEEE Transactions on Information Theory, vol. 53, pp. 4127-4149, November 2007.

[50] S. Weber and M. Kam, "Computational complexity of outage probability simulations in mobile ad hoc networks," in Proceedings of the 39th Annual Conference on Information Sciences and Systems (CISS'05), Baltimore, MD, March 2005.

[51] S. Weber, X. Yang, J. G. Andrews, and G. de Veciana, "Transmission capacity of wireless ad hoc networks with outage constraints," IEEE Transactions on Information Theory, vol. 51, pp. 4091-4102, December 2005.

[52] M. Westcott, "The probability generating functional," The Journal of the Australian Mathematical Society, vol. 14, pp. 448-466, 1972.

[53] X. Yang and G. de Veciana, "Inducing multiscale clustering using multistage MAC contention in CDMA ad hoc networks," IEEE/ACM Transactions on Networking, vol. 15, pp. 1387-1400, December 2007.

[54] M. Zorzi and S. Pupolin, "Optimum transmission ranges in multihop packet radio networks in the presence of fading," IEEE Transactions on Communications, vol. 43, pp. 2201-2205, July 1995.

[55] I. J. Zucker, "Exact results for some lattice sums in 2, 4, 6 and 8 dimensions," Journal of Physics A: Mathematical and General, vol. 7, pp. 1568-1575, 1974. 